\title{
Polyclonal and convergent antibody response to Ebola virus vaccine rVSV-ZEBOV
}

\author{
Stefanie A. Ehrhardt ${ }^{1,16}$, Matthias Zehner ${ }^{1,16}$, Verena Krähling ${ }^{2,3}$, Hadas Cohen-Dvashi ${ }^{4}$, \\ Christoph Kreer', Nadav Elad' ${ }^{5}$, Henning Gruell ${ }^{1,6}$, Meryem S. Ercanoglu', Philipp Schommers ${ }^{1,6,7}$, \\ Lutz Gieselmann ${ }^{1}$, Ralf Eggeling ${ }^{8}$, Christine Dahlke ${ }^{9,10,11}$, Timo Wolf ${ }^{12}$, Nico Pfeifer ${ }^{8,13,14}$, \\ Marylyn M. Addo ${ }^{9,10,11}$, Ron Diskin ${ }^{4}$, Stephan Becker ${ }^{2,3}$ and Florian Klein ${ }^{1,6,15 \star}$
}

\begin{abstract}
Recombinant vesicular stomatitis virus-Zaire Ebola virus (rVSV-ZEBOV) is the most advanced Ebola virus vaccine candidate and is currently being used to combat the outbreak of Ebola virus disease (EVD) in the Democratic Republic of the Congo (DRC). Here we examine the humoral immune response in a subset of human volunteers enrolled in a phase 1 rVSV-ZEBOV vaccination trial by performing comprehensive single B cell and electron microscopy structure analyses. Four studied vaccinees show polyclonal, yet reproducible and convergent B cell responses with shared sequence characteristics. EBOV-targeting antibodies cross-react with other Ebolavirus species, and detailed epitope mapping revealed overlapping target epitopes with antibodies isolated from EVD survivors. Moreover, in all vaccinees, we detected highly potent EBOV-neutralizing antibodies with activities comparable or superior to the monoclonal antibodies currently used in clinical trials. These include antibodies combining the IGHV3-15/IGLV1-40 immunoglobulin gene segments that were identified in all investigated individuals. Our findings will help to evaluate and direct current and future vaccination strategies and offer opportunities for novel EVD therapies.
\end{abstract}

E bola viruses can cause severe infections in humans, leading to unpredictable epidemics with mortality rates of up to $90 \%{ }^{1}$. The devastating outbreak in West Africa in 2013-2016 caused $>11,000$ confirmed deaths ${ }^{2}$. The severe and ongoing outbreak in the Democratic Republic of the Congo (DRC) highlights the significant impact of Ebola virus disease (EVD) and the critical need for efficacious countermeasures ${ }^{3,4}$.

Different Ebolavirus species can cause symptomatic infection in humans. These include Sudan ebolavirus (SUDV), Bundibugyo ebolavirus (BDBV) and Zaire ebolavirus (EBOV) $)^{5-7}$, with the latter accounting for the current outbreak in the $\mathrm{DRC}^{1,8}$. Studies in animal models have demonstrated that neutralizing antibodies can prevent infection and are effective for post-exposure prophylaxis ${ }^{9-17}$. Therefore, monoclonal antibodies have been evaluated in clinical trials and administered to patients suffering from $\mathrm{EVD}^{18-22}$. For example, a combination of three antibodies obtained from immunized mice (REGN-EB3) ${ }^{9}$ has been tested in a phase I clinical trial ${ }^{21}$. Moreover, the neutralizing antibody mAb114 that was isolated from an EVD survivor ${ }^{23}$ has been tested in a clinical trial and demonstrated a preferential pharmacokinetic and safety profile ${ }^{20}$. Until recently, efficacy data in humans were mostly limited to the ZMapp cocktail, which comprises three human-mouse chimeric antibodies (13C6, C2G4 and C4G7). During the 2013-2016 epidemic, ZMapp was tested in EVD patients and reduced fatality rates from $37 \%$ to $22 \%{ }^{22}$. More promising results have been reported for REGN-EB3 and mAb114, which were administered to EVD patients in a multicenter, randomized controlled clinical trial. In an interim analysis from 499 participants, lethal outcome was markedly reduced from $67 \%$ to $29 \%$ and $34 \%$ with REGN-EB3 and mAb114, respectively ${ }^{24}$.

In addition to passive immunotherapy, several vaccine candidates have been developed ${ }^{25-28}$. Among them, the recombinant vesicular stomatitis virus (VSV)-based vector carrying the EBOV glycoprotein (rVSV-ZEBOV) is the most advanced vaccine and has been administered to more than 180,000 individuals ${ }^{24,27,29}$. rVSV-ZEBOV has been demonstrated to be protective against lethal EBOV challenges in rodents and non-human primates (NHPs) ${ }^{13,30,31}$. Moreover, the results of two ring vaccination trials estimate vaccine efficiency to be $97 \%$, and no fatal EVD cases occurred 10 days post vaccination ${ }^{27,32}$. As a vaccine candidate with available efficacy data, rVSV-ZEBOV has been designated as the lead candidate for administration in current and future EVD outbreaks ${ }^{33,34}$. However, despite its broad application, a detailed understanding of the rVSV-ZEBOV immune response is still limited and no single-cell analysis has been performed to elucidate the molecular composition of the induced antibody response. Therefore, we set out to investigate the humoral immune response to rVSV-ZEBOV immunization on a molecular level.

\footnotetext{
'Laboratory of Experimental Immunology, Institute of Virology, Faculty of Medicine and University Hospital of Cologne, University of Cologne, Cologne, Germany. ${ }^{2}$ Institute of Virology, Faculty of Medicine, Philipps University Marburg, Marburg, Germany. ${ }^{3}$ German Center for Infection Research, Partner Site Gießen-Marburg-Langen, Marburg, Germany. ${ }^{4}$ Department of Structural Biology, Weizmann Institute of Science, Rehovot, Israel. ${ }^{5}$ Department of Chemical Research Support, Weizmann Institute of Science, Rehovot, Israel. ${ }^{6}$ German Center for Infection Research, Partner Site Bonn-Cologne, Cologne, Germany. ${ }^{7}$ Department I of Internal Medicine, Faculty of Medicine and University Hospital Cologne, University of Cologne, Cologne, Germany. ${ }^{8}$ Methods in Medical Informatics, Department of Computer Science, University of Tübingen, Tübingen, Germany. ${ }^{9}$ Division of Infectious Diseases, First Department of Medicine, University Medical Center Hamburg-Eppendorf, Hamburg, Germany. ${ }^{10}$ Department of Clinical Immunology of Infectious Diseases, Bernhard Nocht Institute for Tropical Medicine, Hamburg, Germany. "German Center for Infection Research, Partner Site Hamburg-Lübeck-Borstel-Riems, Hamburg, Germany. ${ }^{12}$ Department of Infectious Diseases, University Hospital Frankfurt, Frankfurt/Main, Germany. ${ }^{13}$ Faculty of Medicine, University of Tübingen, Tübingen, Germany. ${ }^{14}$ German Center for Infection Research, Partner Site Tübingen, Tübingen, Germany. ${ }^{15} \mathrm{Center}$ for Molecular Medicine Cologne (CMMC), University Hospital of Cologne and University of Cologne, Cologne, Germany. ${ }^{16}$ These authors contributed equally: Stefanie A. Ehrhardt, Matthias Zehner. *e-mail: florian.klein@uk-koeln.de
} 
a

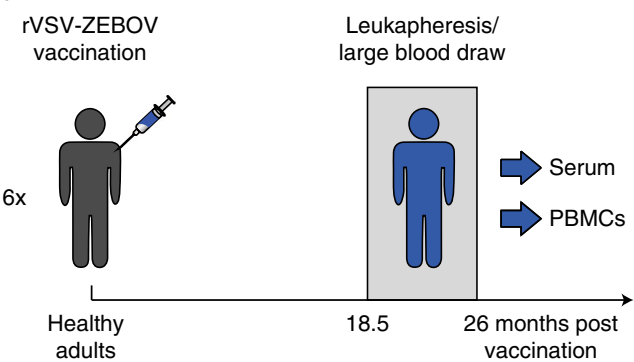

b

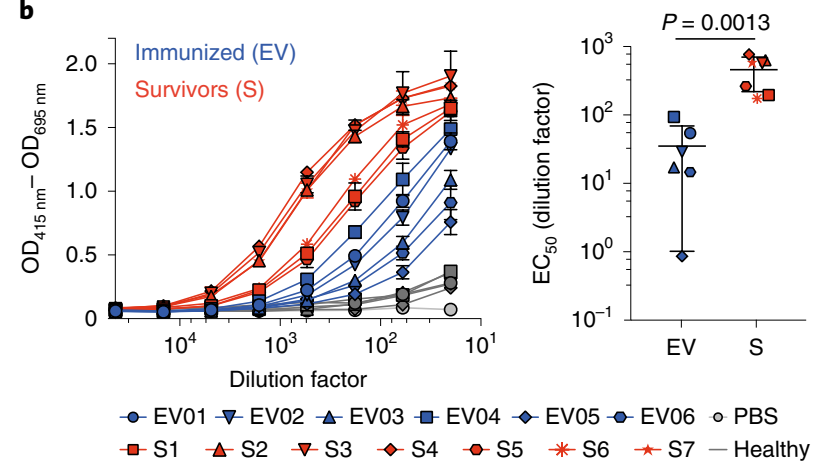

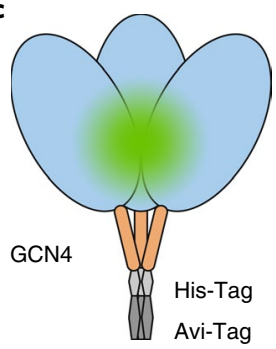

EBOV GP $\Delta T M$

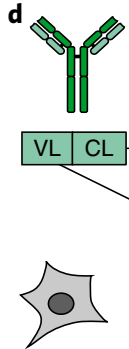

HEK293T 6x EBOV GP.

specific mAbs

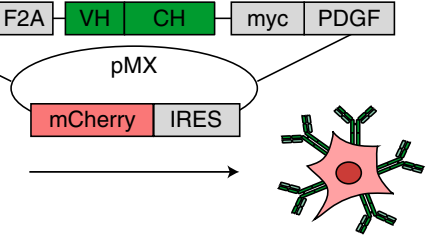

EBOV GP-specific Ab surface expression e

Control

mAb100

mAb114

ADI-15758

ADI-15999

ADI-16037

KZ52

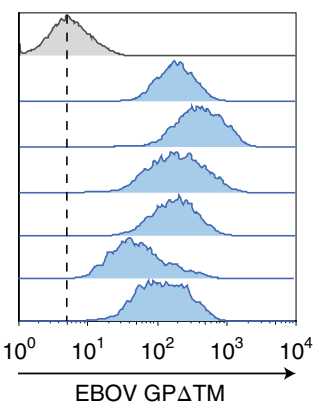

Fig. 1 | Sample collection of rVSV-ZEBOV-vaccinated individuals and design of EBOV GP construct for single B cell analysis. $\mathbf{a}$, Sample collection from rVSV-ZEBOV-immunized donors (EV01-EV06) was performed between 18.5 to 26 months after single dose vaccination. Serum was collected and PBMC samples were obtained after leukapheresis or large blood draw. b, Serum reactivity to EBOV GPATM (Makona) measured by ELISA in rVSV-ZEBOVimmunized subjects (blue) compared to EVD survivors (S1-S7, red, left panel). Error bars show s.d. of means of technical duplicates. Lines indicate means of calculated half-maximum effective concentration $\left(E C_{50}\right.$ ) dilution factors, which are 35 and 456 for vaccinees and survivors, respectively $(n=6$ ( $E V$ ) and 7 (S) independent samples, $P=0.0013$, right panel). PBS and healthy serum samples were used as negative controls. Significance was tested by a two-tailed unpaired $t$-test. c, Design of EBOV GP $\triangle$ TM construct with GCN4 trimerization domain, His- and Avi-Tag, as well as covalent labeling with fluorophore DyLight 488 (green). d, Generation of constructs for surface expression of EBOV GP-reactive antibodies (mAb100, mAb114, ADI-15758, ADI-15999, ADI-16037 and KZ52) on HEK293T cells. Variable and constant regions of immunoglobulin (lg) light (VL, CL) and heavy chains (VH, CH) were linked by a F2A self-cleaving signal sequence followed by a c-myc, a platelet-derived growth factor (PDGF)-transmembrane domain, an internal ribosomal entry site (IRES) and mCherry. As control, an empty vector ( $\mathrm{PMX}$ ) containing only an IRES-mCherry was used. e, Flow cytometry analysis to evaluate EBOV GPATM recognition by antibody-expressing HEK293T cells as a surrogate for anti-EBOV GPATM-reactive B cells (blue areas). The dashed line indicates the peak fluorescence intensity of no antibody vector control. See also Supplementary Table 1. Data represent the results of $n=4$ independent experiments.

\section{Results}

Deciphering the rVSV-ZEBOV-specific B cell response. To analyze the human B cell response to rVSV-ZEBOV vaccination, we collected serum and peripheral blood mononuclear cell (PBMC) samples from six healthy individuals at 18.5 to 26 months after vaccination (Fig. 1a). The age of participants ranged from 26 to 55 years (Supplementary Table 1 ) and subjects were vaccinated with $3 \times 10^{5}$ (EV01, EV02, EV04) or $3 \times 10^{6}$ (EV03, EV05, EV06) plaqueforming units (p.f.u.) of rVSV-ZEBOV (single intramuscular injection). Sera of all participants bound to EBOV glycoprotein (GP) in ELISAs, but with a significantly $(P=0.0013)$ lower activity than serum samples of EVD survivors (Fig. 1b). Less to almost no reactivity was detected against GPs from representative viruses of other Ebolavirus species (BDBV and SUDV) and Marburg virus (MARV; Extended Data Fig. 1).

To analyze the rVSV-ZEBOV-induced antibody response on a molecular level, we cloned and produced the EBOV GP ectodomain ( $\Delta 651-676)$, introduced a trimerization domain (GCN4 $\left.4^{23}\right)$ and tags for purification and labeling (His- and Avi-Tag), and conjugated the resulting protein to a fluorescence marker (the entire construct is referred to as EBOV GP lacking the transmembrane domain ((EBOV GPATM), Fig. 1c). To determine whether EBOV GP $\Delta$ TM identifies EBOV GP-specific antibodies on cell surfaces, six GP-specific antibodies that have been reported before were cloned into surface expression vectors and expressed on HEK293T cells as a surrogate for GP-specific B cells (Fig. 1d). Antibodies recognized different GP epitopes including a discontinuous epitope of base and internal fusion loop (IFL; mAb100). Others bridged GP1 and GP2 (KZ52), bound the GP1 core (mAb114), the GP2 stalk (ADI-15758, ADI-15999) or the glycan cap (ADI-16037). HEK293T cells carrying each of the GP-specific antibodies could be detected by FACS analysis after staining with EBOV GP $\triangle \mathrm{TM}$ (Fig. 1e). We conclude that EBOV GP $\triangle \mathrm{TM}$ is able to identify anti-EBOV GP $\Delta \mathrm{TM}$ antibodies that recognize various epitopes when expressed on cells and is therefore a suitable protein to isolate EBOV GP-specific B cells and to study the total anti-EBOV GP B cell response in humans.

In six rVSV-ZEBOV-vaccinated subjects, FACS analysis revealed between $0.29 \%$ and $0.67 \%$ EBOV GP $\Delta$ TM-reactive $\mathrm{IgG}^{+} \mathrm{B}$ cells (Fig. 2a and Extended Data Fig. 2a,b). Four subjects vaccinated with either $3 \times 10^{5}$ (EV01, EV04) or $3 \times 10^{6}$ p.f.u. (EV03, EV05) rVSVZEBOV were selected for in-depth single B cell and Ig sequence analysis. To this end, we applied nested PCR protocols using highly effective primer sets (Kreer et al., submitted). After quality checks, 1,507 Ig heavy- $\left(\mathrm{V}_{\mathrm{H}}\right)$ and 261 light-chain variable $\left(\mathrm{V}_{\mathrm{k} / \lambda}\right)$ regions of individual $\mathrm{B}$ cells were closely evaluated. Analysis of $\mathrm{V}_{\mathrm{H}}$ regions revealed clonal origins for $33-46 \%$ of sequences (Fig. 2b). Among these sequences, we detected a polyclonal response in all subjects, identifying 49, 42, 51 and 45 individual B cell clones for EV01, EV03, EV04 and EV05, respectively (Fig. 2c and Supplementary Table 2). The distribution of sequenced heavy (IgH) and light 
chain (IgL) V gene families was comparable among all vaccinees (Fig. 2d), and the mean germline identity ranged from $90.6 \%$ to $94.2 \%$ for heavy and $93.9 \%$ to $95.9 \%$ for light chains (Fig. 2e). In addition, only minor differences were detected for the average CDR3 length in amino acids (aa) of $\operatorname{IgH}(13-15 \mathrm{aa})$ and $\operatorname{IgL}(10-11 \mathrm{aa}$; Fig. 2e) among vaccinees. Notably, very similar results were observed in an individual (EV07) that received $2 \times 10^{7}$ p.f.u. (Extended Data Fig. 3a-e) and when using an alternative bait lacking the TM and mucin-like domain (MLD) (EBOV Makona GP $\Delta$ TM $\Delta$ MLD) (Extended Data Fig. 4a,b). We conclude that rVSV-ZEBOV elicits a polyclonal B cell response that shows similar characteristics among vaccinees, including $\mathrm{V}$ gene distribution, CDR3 lengths and levels of somatic hypermutation (SHM).

Finally, we compared the EBOV GP $\Delta$ TM-specific response to the overall B cell repertoire of the same donors (EV01, EV03, EV04 and EV05). To this end, we performed unbiased next generation sequencing (NGS) analyses on heavy chains of the memory B cell compartment of the vaccinees (Extended Data Fig. 2c). Differences of CDRH3 lengths and SHM between GP-reactive B cells (blue) and the donor-matched B cell memory compartment (gray) were small, with differences ranging from 0.8 to 1.3 aa for the mean CDRH3 lengths and -0.9 to $1.0 \%$ for mean $\mathrm{V}$ gene germline identity (Fig. 2f). However, a striking increase in frequency was detected for the variable gene segment IGHV3-15 (Fig. 2g). Indeed, while IGHV3-15 was detected with an average frequency of $1.3 \%$ in the memory B cell compartment, we detected a significant 5.4-, 6.4-, 4.8- and 9.9-fold increase for EV01, EV03, EV04 and EV05, respectively. Therefore, we conclude that, while CDRH3 lengths and SHM values are similar between the total memory $\mathrm{B}$ cell repertoire and the EBOV GP-specific population, there is a significant increase of IGHV3-15 expressing B cells in the EBOV GP-specific population.

rVSV-ZEBOV induces a convergent B cell response with shared sequence characteristics. To further investigate the level of convergence in the rVSV-ZEBOV-induced B cell response, we compared heavy chain sequences with the same $V_{H}$ gene among the four individuals studied. By applying a cutoff for the CDRH3 identity of at least $75 \%$, we identified 14 groups that comprised sequences from two or more distinct vaccinees (Fig. 3a and Supplementary Table 3). Within these groups, identical or chemically related heavy and light chain CDR3s across different donors were found (Fig. 3a). Of note, we observed antibodies with the same characteristics after vaccination with $2 \times 10^{7}$ p.f.u. (Extended Data Fig. 3f). Quantification of CDRH3 similarities among antibodies within groups that share $\mathrm{CDRH} 3$ characteristics revealed a high degree of homology ranging from $74.8 \%$ to $95.0 \%$ average sequence concordance within groups (Fig. 3b). Additionally, when integrating a set of sequences obtained from EVD survivors ${ }^{14}$, shared characteristics could also be identified between vaccinees and EVD survivors (Extended Data Fig. 5a,b). Additionally, we plotted the normalized Levenshtein distances of CDRH3 amino acids ( $x$ axis, Fig. 3c) and the variable regions
( $y$ axis, Fig. 3c) of EBOV GP-specific antibodies. Shared groups are displayed by colored antibodies and cluster in the lower left part of the diagram. Of note, when performing the same analysis on HIV-1 envelope-reactive antibodies, no shared antibody groups were identified. Taken together, these data indicate a convergent antibody response within rVSV-ZEBOV-vaccinated donors (Fig. 3) as well as similarities to the specific antibody response upon EBOV infection (Extended Data Fig. 5).

rVSV-ZEBOV-induced antibodies are cross-reactive and target a broad spectrum of epitopes. To produce a broad spectrum of EBOV-directed antibodies, we selected sequences from identified B cell clones as well as a limited number of non-clonal (single) antibody sequences. In total, we cloned and produced 143 antibodies, of which 125 represented members of individual B cell clones and 18 were non-clonal antibodies. Of all 143 antibodies, binding against EBOV GP $\triangle \mathrm{TM}$ was detected in $55-74 \%$. Calculated $\mathrm{EC}_{50}$ values ranged from 0.03 to $14.4 \mu \mathrm{g} \mathrm{m}^{-1}$ (Fig. 4a) and all EBOV GP $\Delta$ TM-specific antibodies were also reactive to strain Mayinga (Supplementary Table 4). In addition, a large fraction (30.4-75.9\%) of antibodies were cross-reactive, with an average of $42.5 \%$ recognizing GPs of EBOV, BDBV and SUDV (Fig. $4 \mathrm{~b}$ and Supplementary Table 4). Interestingly, four antibodies detected MARV with a mean $\mathrm{EC}_{50}$ of $4.4 \mu \mathrm{g} \mathrm{ml}^{-1}$ (Fig. $4 \mathrm{~b}$ ).

Next, we determined the epitopes of reactive antibodies using several EBOV GP constructs (the trimeric GP $\Delta \mathrm{TM}$ and $\mathrm{GP} \Delta \mathrm{TM} \Delta \mathrm{MLD}$, as well as the structurally different and dimeric secreted GP (sGP), Extended Data Fig. 6a). For EV01, EV03, EV04 and EV05, 28.6, 47.8, 34.5 and $68.8 \%$ of antibodies targeted sGP, respectively, and in $6.3-33.3 \%$ binding was dependent on the presence of the MLD (Fig. 4c). Meanwhile, $14.3-66.7 \%$ of EBOV GP $\Delta$ TM-reactive antibodies competed with either mAb100 or KZ52, which were shown to recognize epitopes on the glycan cap and loop structure ${ }^{23,35}$. To a lower extent (up to 16.7\%), antibodies competed with ADI-15758 and ADI-15999, which target the MPER of GP2 ${ }^{14}$ (Fig. 4d, Extended Data Fig. 6b and Supplementary Table 4). To complement epitope mapping, we assessed binding to linear epitopes for all EBOV Makona GP $\Delta$ TM-reactive $m A b s$ using a peptide library (Extended Data Fig. 7a and Supplementary Tables 4 and 5). The library was validated using control antibodies including KZ52, mAb100, mAb114 and ADI-15758 (Extended Data Fig. 7a). Of all the antibodies, 52 detected at least one linear peptide, confirming and refining epitope information (Supplementary Table 5). In summary, $49.4 \%$ of EBOV GP $\Delta$ TM-reactive antibodies interacted with the N-terminal part of GP1 (sGP) and $16.5 \%$ with the MLD. In addition, $7 \%$ of the antibodies were directed against the GP2 and 12.9\% against GP1/GP2 (Fig. 4e and Supplementary Tables 4 and 5). Strikingly, the epitope distribution closely overlapped with epitopes of a collection of antibodies recently characterized in a comprehensive study by Saphire and colleagues ${ }^{36}$ (Fig. 4e). This set of antibodies comprised antibodies from human survivors and

Fig. 2 | Deciphering the EBOV GP-specific B cell response. a, Flow cytometry on CD19+ magnetic bead-enriched PBMCs from four rVSV-ZEBOV-vaccinated individuals (EV01, EV03-EV05). One of two independent experiments with similar results is shown. Gates capture the EBOV GP $\triangle T$ TM-reactive populations from $\mathrm{IgG}^{+} / \mathrm{CD} \mathrm{O}^{+} \mathrm{B}$ cells that were selected for single cell sorting. $\mathbf{b}$, Abundance of amplified clonal (dark blue) and non-clonal (light blue) Ig sequences. c, Identified B cell clonotypes (the total number is shown in the center of the pie charts) for each vaccinated individual. Each slice represents a unique B cell clone (size proportional to the number of clonal members). d, Distribution of $V$ gene families in heavy $(H$, top) and light $(L, \kappa / \lambda$, bottom) chains of all unique clones. e, Germline identity (left) and CDR3 amino acid lengths (right) of $V_{H}$ and $V_{k / \lambda}$ of all clonal sequences identified in $\mathbf{b}$ ( $n=339,381,412$ and 375 for EV01, EV03, EVO4 and EV05, respectively). Means are shown in red and s.d. in black. f, Matched comparison of sequence characteristics between EBOV GPATM-specific B cells (blue) and the overall memory B cell repertoire (gray) within individuals. Heavy chain CDR3 amino acid length (top) and $\checkmark$ gene segment identity (bottom) are shown. Mean amino acid lengths and identities are indicated $(\varnothing)$. $\mathbf{g}$, Differences in frequencies of heavy chain $V$ gene segments between B cell lgG repertoire and EBOV GP $\Delta$ TM-specific lgG ${ }^{+} / C D 20^{+} B$ cells from $n=4$ independent samples of $r V S V$-ZEBOV-immunized individuals. Boxes contain $50 \%$ of available data points and whiskers indicate $75 \%$ of the difference between the highest or lowest value. Lines within boxes display median values. The significance of frequency increase was tested by a resampling approach based on a one-sided Mann-Whitney $U$-test with $\alpha=0.05$ and Bonferroni correction for multiple testing. See also Extended Data Fig. 2 and Supplementary Tables 2 and 5. 
antibodies from animal models induced by vaccination or viral challenge. Additionally, a separate epitope analysis of cross-reactive antibodies revealed that $47 \%$ of detected epitopes were present on
sGP (Extended Data Fig. 7b). Notably, while only a small fraction of the MLD-detecting antibodies (4 of 13) were cross-reactive, nearly all GP2 binders showed cross-reactivity (5 of 6 , Supplementary
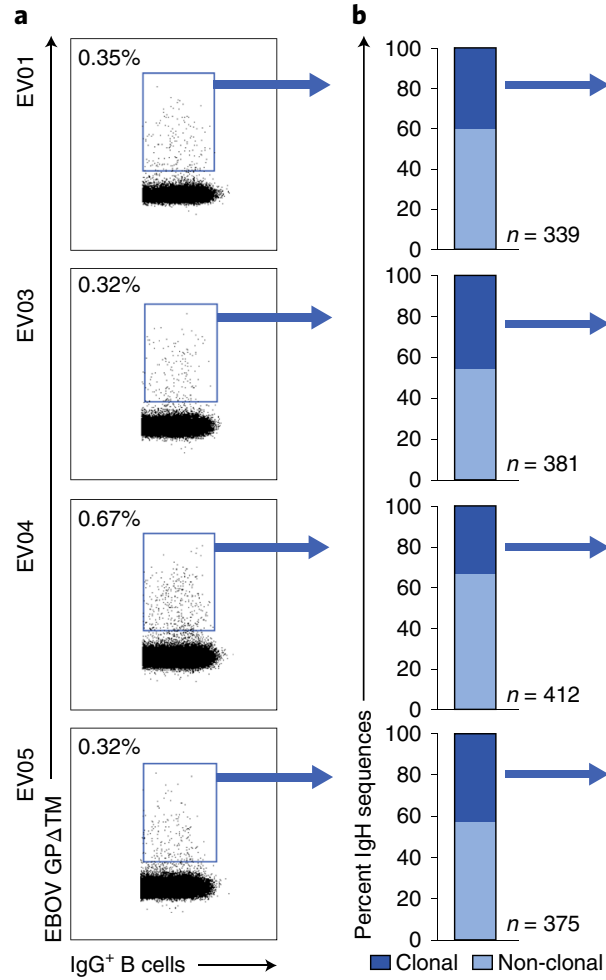

c
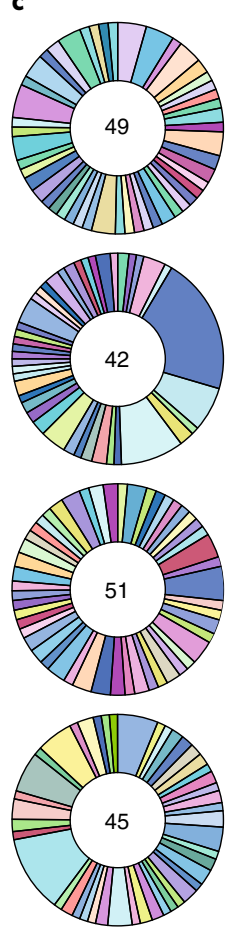

B cell clones d
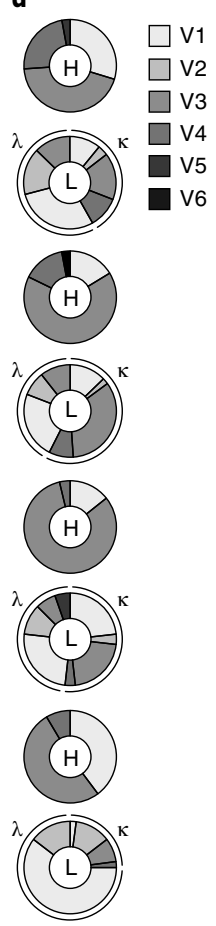

$\uparrow$

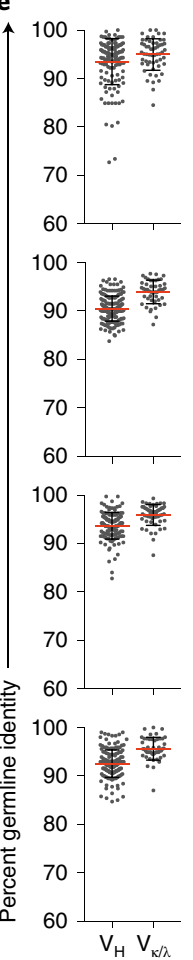

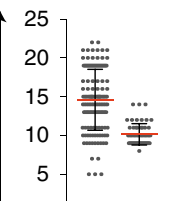

0

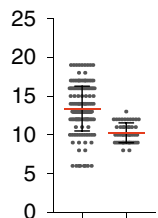

25

$20]$

$\begin{aligned} & 20 \\ & 15 \\ & 10\end{aligned}-$ 爯

$5-$

0

25

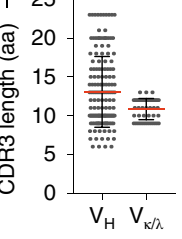

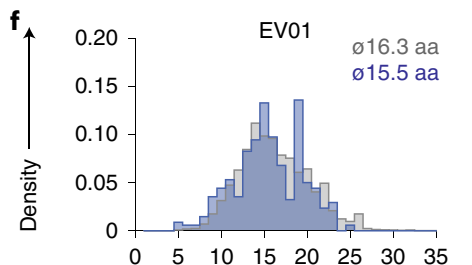
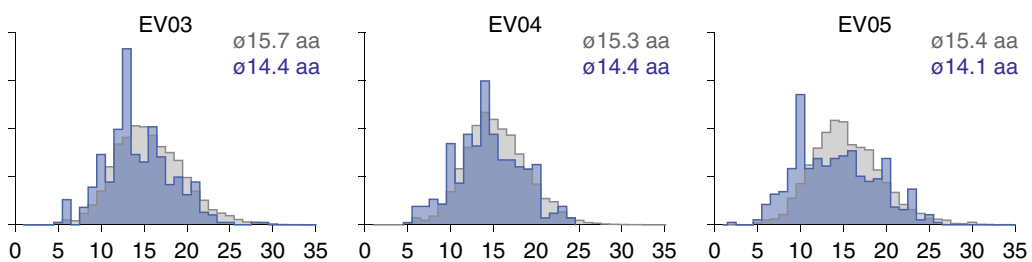
CDR3 length (aa)
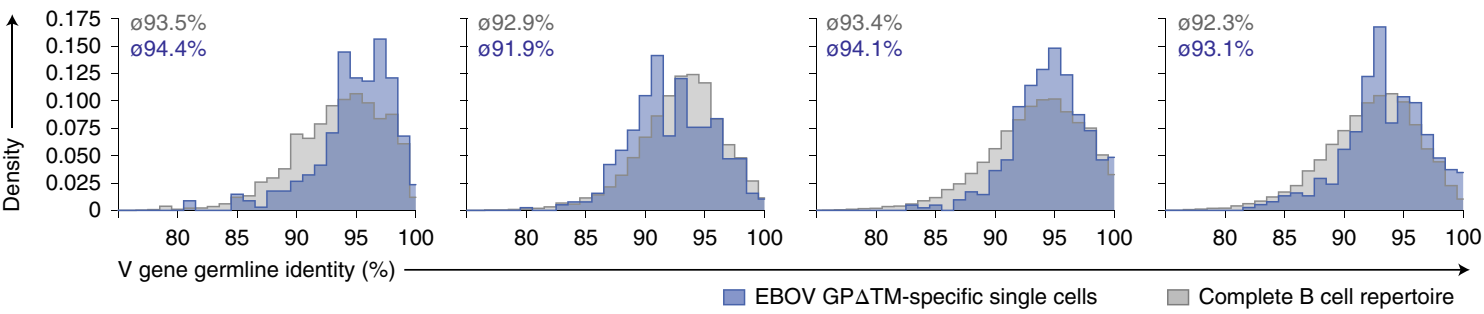

g

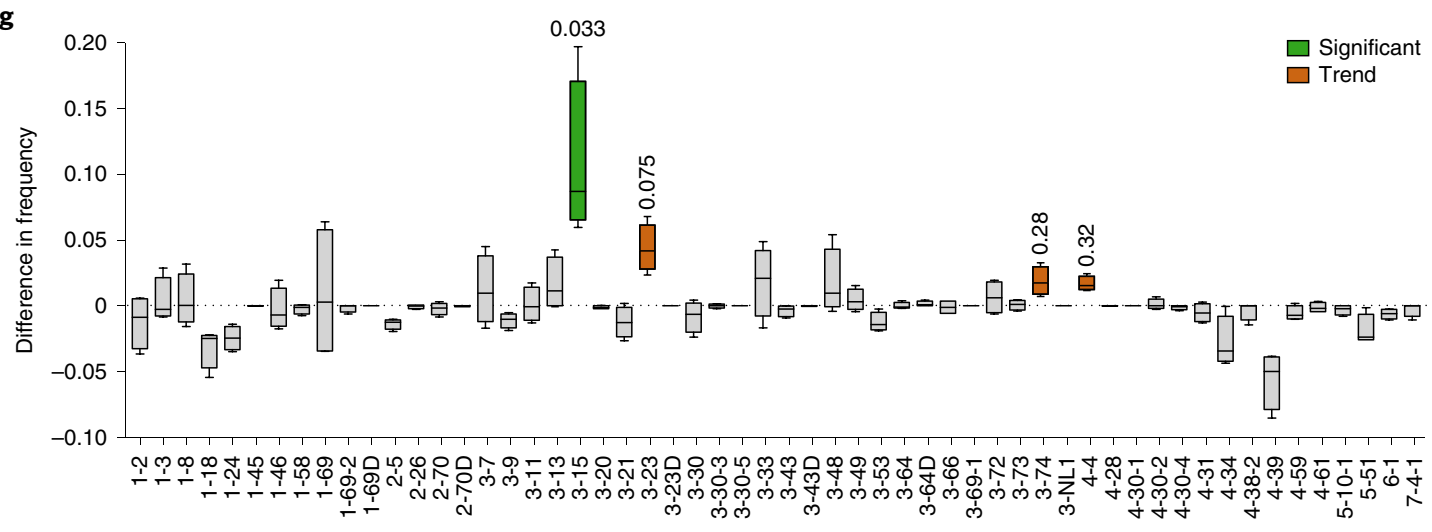




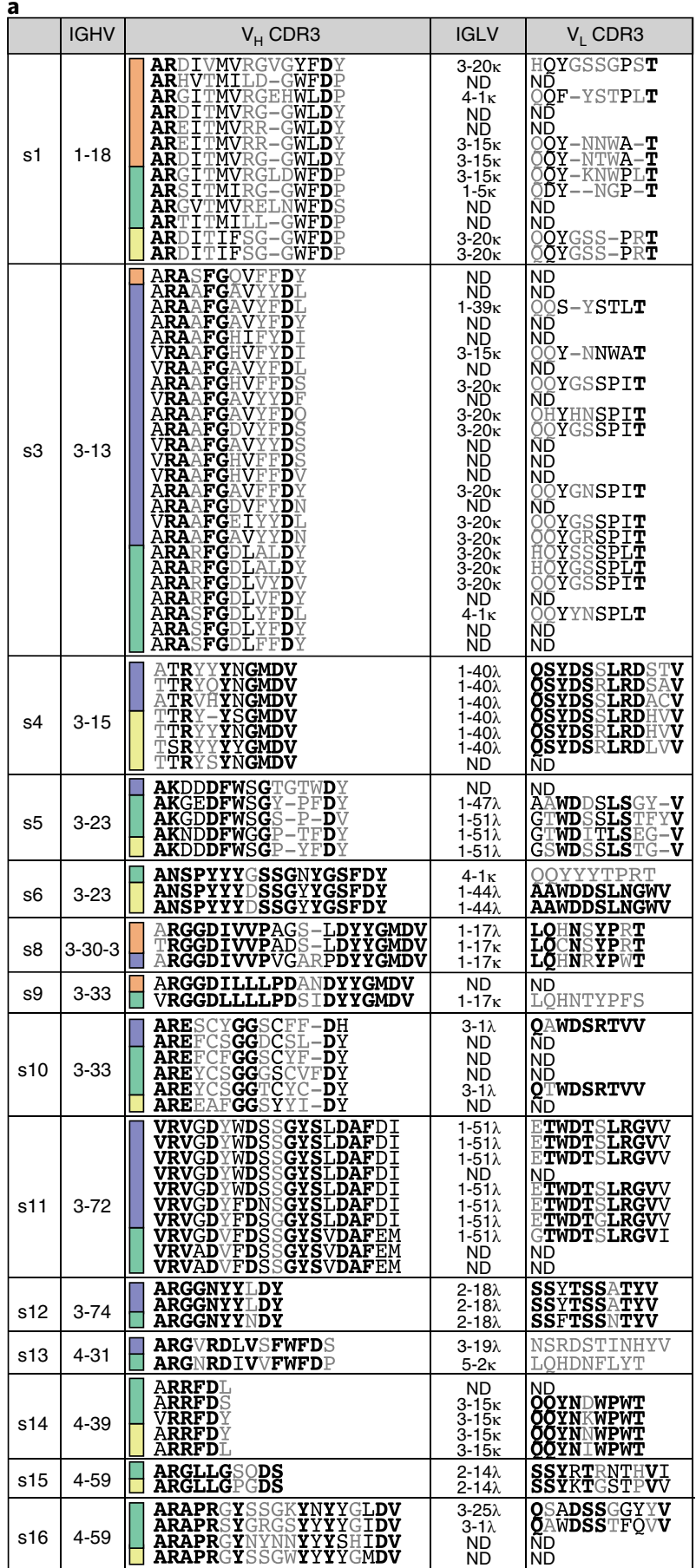

$\square$ EV01 $\square$ EV03 $\square$ EV04 $\square$ EV05 - Gap

Amino acid similarity: oIdentical amino acids

- Similar amino acids (similar chemical characteristics)

- Different amino acids
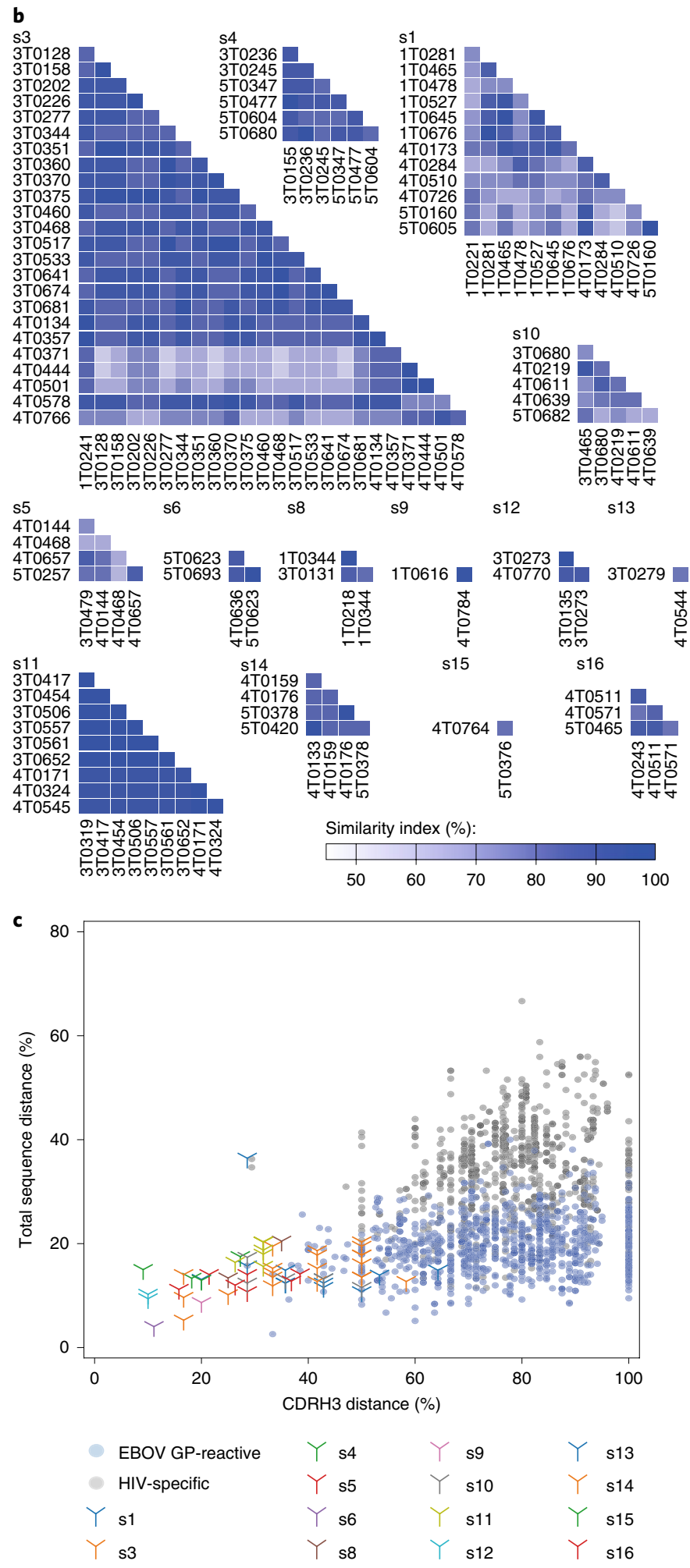

Fig. 3 | Convergent development of EBOV GPATM-specific antibody sequences. $\mathbf{a}$, Heavy chain sequences of all vaccinees with the same $V$ gene were grouped and tested for their similarity on the amino acid level by calculating the Levenshtein distance. Sequences from different donors with at least $75 \%$ $\mathrm{CDRH} 3$ homology were defined as a shared group (s1-s16) and, if available, clonal members were added to groups. Sequence origins are color-coded orange, violet, green and yellow for EV01, EV03, EV04 and EV05, respectively. Bold black letters indicate identical amino acids, black letters amino acids with similar chemical characteristics and gray letters different amino acids. 'ND' indicates, where genes or CDR3 domains were not defined. Chemical characteristics were defined by grouping amino acids into non-polar, polar, acidic, basic and aromatic amino acids. $\mathbf{b}$, Quantification of homology of CDRH3 sequences by similarity index. The index was determined using BLOSUM (BLOcks SUbstitution Matrix). c, Percent distance of CDRH3 ( $x$ axis) and total sequence distance ( $y$ axis) of each sequence to the most similar sequence identified in another donor as calculated by Levenshtein distance normalized on the shorter sequence, if applicable. EBOV GP-reactive antibodies are shown in blue and shared EBOV-specific groups with at least 75\% CDRH3 homology in other colors. BG505. SOSIP-reactive antibodies from $n=6 \mathrm{HIV}$-1-infected individuals are labeled as gray dots. See also Extended Data Figs. 3 and 5 and Supplementary Table 3. 
Table 4). Interestingly, similar epitope distributions were found in antibodies published in refs. ${ }^{14,15}$ (Extended Data Fig. 7c). These data illustrate that rVSV-ZEBOV induces a reproducible antibody response that is cross-reactive and targets a broad spectrum of epitopes. Moreover, the rVSV-ZEBOV-induced antibody response recapitulates the epitope distribution found in previously identified antibodies, including those from EVD survivors.

rVSV-ZEBOV-induced mAbs demonstrate potent neutralizing activity. To determine the neutralizing activity of rVSV-ZEBOVinduced antibodies, we performed neutralization assays with wild-type EBOV and SUDV. On serum level, full neutralization of EBOV was observed at higher concentrations in vaccinees (average titer of 1:30) compared to EVD survivors (average titer of 1:104; Fig. 4f). However, we observed that $34.5-65.2 \%$ of rVSV-ZEBOVinduced and EBOV GP-specific antibodies displayed EBOVneutralizing activity (Fig. 4g), but no cross-neutralization of SUDV (Supplementary Table 4). Nearly 70\% of antibodies were found to neutralize within the same range (full neutralization at $0.055-$ $2.5 \mu \mathrm{g} \mathrm{ml}^{-1}$ ) as control antibodies that have been evaluated in clinical trials $(\mathrm{mAb} 114)^{20}$ and/or showed protection in NHPs (mAb100, ADI-15758, ADI-15999, ADI-16037) $)^{10,14,23}$. Moreover, some antibodies demonstrated exceptional neutralizing activity by fully preventing infection in vitro, even at $0.01 \mu \mathrm{g} \mathrm{ml}^{-1}$ (3T0331 and 4T0243, Fig. 4h). Although epitopes of most neutralizing antibodies were mapped to sGP, various other epitopes were also recognized (Fig. 4i). Of all neutralizing antibodies, four targeted the MLD, two competed with ADI-15758 and ADI-15999, and six competed with mAb100 and KZ52. The two most potent neutralizers, 3T0331 and 4T0243, were competitors of mAb100 and KZ52, but with a 5- and 12-fold higher activity, respectively (Fig. 4h,i and Supplementary Table 4). These data demonstrate that rVSV-ZEBOV induces a broad spectrum of neutralizing antibodies that target different epitopes and are of high potency.

Recurrent generation of a specific EBOV-neutralizing antibody class. Although various $\mathrm{V}$ genes were observed in EBOVneutralizing antibodies, we detected a significant preference for IGHV3-15 in heavy chains and IGLV1-40 in light chains (Fig. 5a). Interestingly, among the 21 antibodies carrying IGHV3-15, 17 showed neutralizing activity ranging from 0.44 to $12.5 \mu \mathrm{g} \mathrm{ml}^{-1}$ and were exclusively paired with IGLV1-40. Cross-competition ELISAs indicate a similar epitope for all IGHV3-15/IGLV1-40 antibodies and all of them competed with mAb114 (Fig. 5d). Of note, this antibody class (IGHV3-15/IGLV1-40) was identified in all four individuals studied (Fig. 5b,c). The remaining four IGHV3-15 antibodies were paired with other light chains and did not show neutralizing activity (Fig. 5b and Supplementary Tables 2 and 6). Notably, this combination was also found frequently in the highdose vaccinee EV07 (11 of 18 analyzed IGHV3-15 antibodies; Extended Data Fig. 3g).

A detailed sequence comparison of IGHV3-15/IGLV1-40 EBOV-neutralizing antibodies revealed that phylogenetic distances among different donors were small and similar to the distances within single donors (Fig. 5c). Moreover, in all IGHV3-15/ IGLV1-40 sequences, a high degree of overlapping mutations was observed, indicating a strongly directed affinity maturation process upon rVSV-ZEBOV vaccination (Fig. 5e and Extended Data Fig. 3h). For example, in IGHV3-15 heavy chains, various positions were consistently mutated. These include mutations rendering the Ser at position 40 to become an Asn or Thr in $88.2 \%$ or within the CDRH1 and CDRH2, in which Ser31, Lys59/Thr60 and Thr64/65 were mutated, in over 50\%. Likewise, in IGLV1-40 light chains, Ser58 (CDRL2) was replaced in $100 \%$ by mostly Asn or Thr. Moreover, within the CDRL3, serines at positions 110 and 112 were mutated to Arg in 82 and 65\%, respectively, and the Gly113 was always mutated to Asp (17 of 17 sequences; Fig. 5e). We conclude that rVSV-ZEBOV can recurrently elicit a class of EBOVneutralizing antibodies that target sGP and utilize the same $\mathrm{V}_{\mathrm{H}}$ and $V_{\lambda}$ genes. Moreover, sequence convergence strongly indicates affinity maturation in a reproducible pattern.

Evaluation and structural analysis of neutralizing mAbs 3T0331 and $4 \mathrm{m0368}$. Antibodies $3 \mathrm{~T} 0331$ and $4 \mathrm{~m} 0368$ demonstrate strong EBOV-neutralizing activities (full neutralization at $0.01 \mu \mathrm{g} \mathrm{ml}^{-1}$ for $3 \mathrm{~T} 0331$ and $0.13 \mu \mathrm{g} \mathrm{ml}^{-1}$ for $4 \mathrm{~m} 0368$; Supplementary Table 4), comparable or superior to the activity of anti-EBOV GP antibodies currently used for clinical application ${ }^{20}$ (Fig. 4h). To elucidate the mechanisms of neutralization on a molecular level, we formed complexes of EBOV GP (Mayinga) with the antigen binding fragment (Fab) of 3T0331 and 4m0368 and determined near-atomic-resolution structures using single-particle cryo-electron microscopy (cryo-EM). EBOV GP/3T0331 and EBOV GP/4m0368 complexes were resolved to $3.1 \AA$ (Fig. 6a and Extended Data Fig. 8a-c) and 3.3 ̊ (Fig. $6 \mathrm{c}$ and Extended Data Fig. 8a-c), respectively. Both structures were modeled by fitting an available crystallographic structure

Fig. 4 | rVSV-ZEBOV-induced antibodies are cross-reactive, target a broad spectrum of different epitopes and show neutralizing activity. a, ELISA binding analysis of mAbs isolated from rVSV-ZEBOV-vaccinated individuals (EV01, EV03-EV05) against EBOV GP $\Delta$ TM ( $n=33$ (EV01), 42 (EV03), 39 (EV04) and 29 (EV05)). Proportion of binding and non-binding mAbs (left) and EC 50 values of binding mAbs (right). b, Percentage of EBOV GPATMspecific mAbs with cross-reactivity against GPs from different filoviruses determined by ELISA ( $n=21$ (EV01), 23 (EV03), 29 (EV04) and 16 (EV05) (left)) and $\mathrm{EC}_{50}$ values ( $n=42$ (BDBV), 32 (SUDV) and 4 (MARV) right). Binding criteria were defined as in a. mAbs specific for GPs of EBOV plus one other filovirus are shown in blue colors and $\mathrm{mAbs}$ specific for GPs of EBOV plus two or three other filovirus species are shown in light or dark red, respectively. In $\mathbf{a}$ and $\mathbf{b}$, means are indicated by lines. c, Percentage of EBOV GPATM-specific antibodies reactive to sGP or MLD determined by ELISA using constructs described in Extended Data Fig. 6a. d, Competition ELISA of EBOV GP $\Delta$ TM-reactive mAbs with target sites not identified in c. Antibodies for competition included KZ52 and mAb100 that target GP1, IFL and heptad repeat 1 (HR1), as well as ADI-15758 and ADI-15999 that target the membrane proximal external region (MPER). e, Summary of epitope distribution determined by ELISA (c,d) and by peptide library screening of antibodies obtained from rVSV-ZEBOV-immunized individuals (right) compared to the target epitope spectrum and abundance in antibodies published in ref. ${ }^{36}$ (left; total numbers are shown in the center of each pie). Unknown epitopes (ND) are shown in gray. See also Extended Data Figs. 4-7 and Supplementary Table 4. f, Analysis of serum neutralizing activity of EVD survivors (red) and rVSV-ZEBOV-immunized donors (EV, blue). Shown are dilution factors yielding full neutralization of EBOV Mayinga. Colored background areas indicate the mean values. Significance was tested by two-tailed unpaired t-tests ( $n=7$ (Survivor) and $n=6$ (EV) biologically independent samples, $P=0.089$ ). $\mathbf{g}$, Proportions of neutralizing (blue) and non-neutralizing (gray)

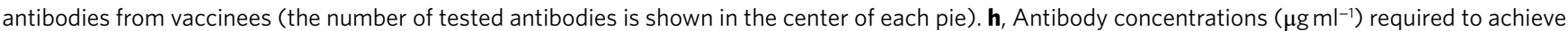
full neutralization. mAbs obtained from rVSV-ZEBOV-immunized subjects (blue; $n=9$ (EV01), 14 (EV03), 10 (EV04), 9 (EV05)) compared to published antibodies (green; KZ52, mAb114, mAb100, ADI-15758, ADI-15999 and ADI-16037). The shaded area illustrates the neutralization range of the reference antibodies. Two mAbs 3T0331 (EV03) and 4T0243 (EV04) are situated below the shaded area. Means are indicated by lines. $\mathbf{i}$, Distribution of neutralizing antibodies by epitopes ( $n=26$ (sGP), 4 (MLD), 2 (ADI-15758-15999), 6 (KZ52/mAb100) and 4 (Others)). Means are indicated for each sample set. Neutralization experiments were performed in quadruplicate. See also Supplementary Table 4. 
of EBOV GP (PDB 5JQ3 ${ }^{37}$ ) into electron density maps. The $\mathrm{V}_{\mathrm{H}} \mathrm{V}_{\mathrm{L}}$ portion of 3T0331 was derived from a $1.6 \AA$ resolution structure of 3T0331-Fab determined by X-ray crystallography (Supplementary Table 7). The $\mathrm{V}_{\mathrm{H}} \mathrm{V}_{\mathrm{L}}$ portion of $4 \mathrm{~m} 0368$ was computationally modeled using ABpredict ${ }^{38}$. In both structures, the interaction surfaces between the mAbs and EBOV GP were resolved to $\sim 3.0 \AA$ or higher (Extended Data Fig. 8a,d,e).

Based on these structures, we revealed that antibody 3T0331 binds to the side of the trimeric EBOV GP with a binding angle that probably positions its Fc portion closely to the viral membrane (Fig. 6a) and with its epitope spanning the $\mathrm{N}$-terminal region of GP2 as well as limited parts of GP1 (Fig. 6b). The main contacts with the EBOV GP are made by the burial of EBOV-Val505 in a hydrophobic pocket, as well as a series of polar contacts that include salt bridges between EBOV-Glu502 and two arginine residues from the heavy and light chains (Arg101 and Arg91, respectively), a hydrogen bond between heavy chain Asn54 and a main chain carbonyl of EBOV, a salt bridge between EBOV-Asp552 and heavy

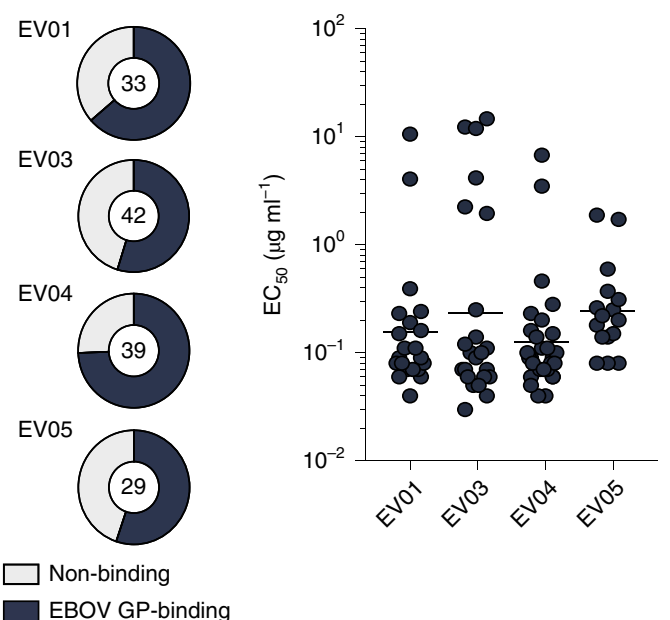

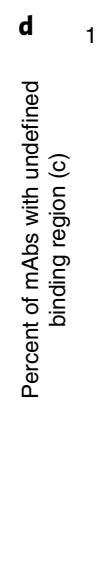

GPs:

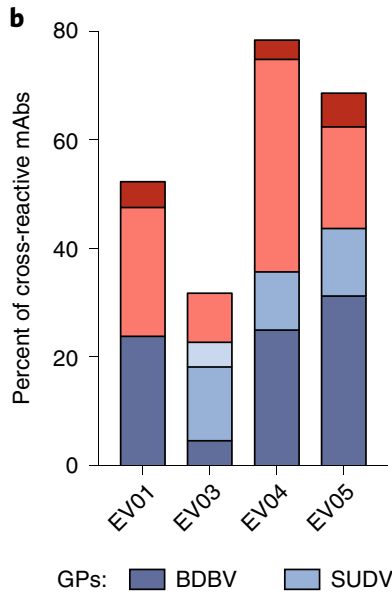

Ps: $\square$ BDBV $\square$ SUDV

BDBV + SUDV + MARV

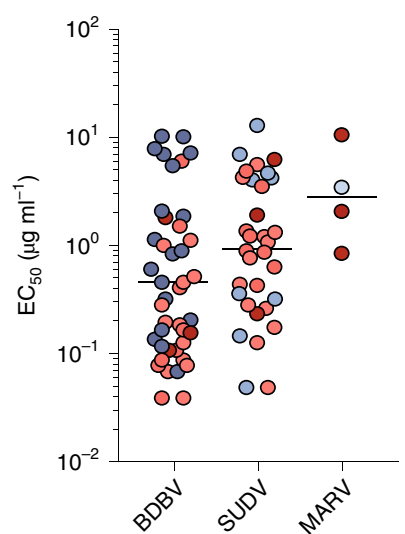

BDBV + SUDV
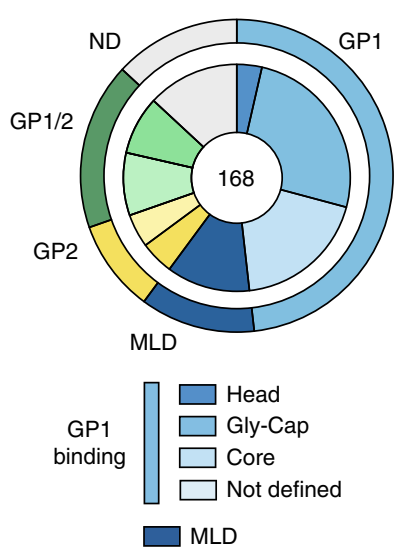

h

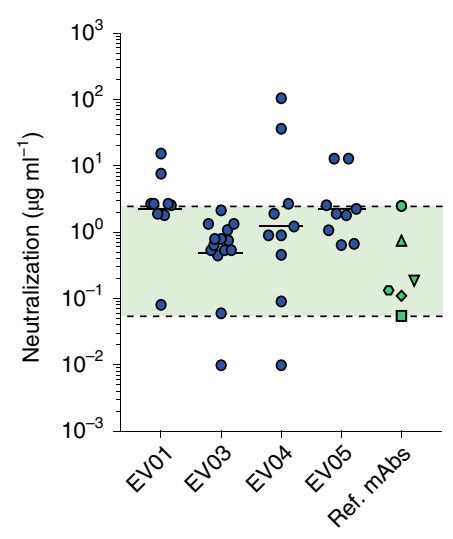

$\nabla$ ADI-15758 ○ ADI-16037 ם mAb100 $\diamond$ ADI-15999 ○ KZ52 $\triangle$ mAb114
rVSV-ZEBOV-induced mAbs

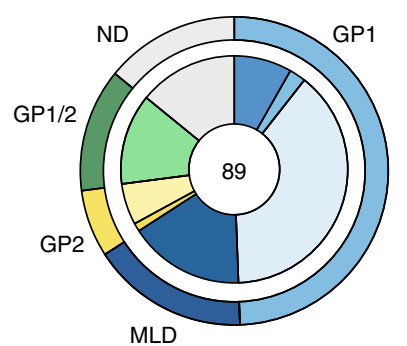

GP2 $\square \mathrm{IFL}$

binding $\square$ HR1 + 2/MPER

GP1/2 $\square$ Base/KZ52 competition binding $\square$ GP1/2 Unknown
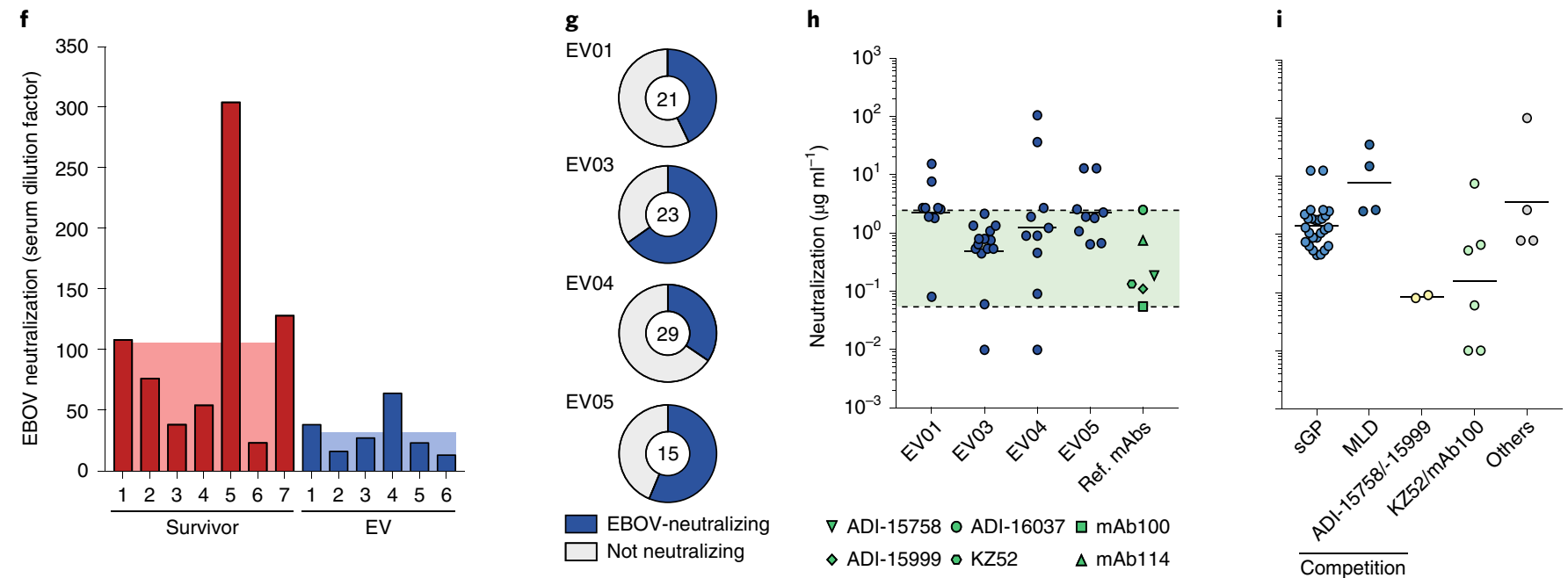
chain Lys65, and a hydrogen bond between EBOV-Arg54 and light chain Gln27 (Fig. 6b).

$4 \mathrm{~m} 0368$, on the other hand, binds the side of the trimeric EBOV GP with an angle that may position its Fc portion further away from the membrane (Fig. 6c). Its epitope is exclusively limited to residues from the GP2 portion as well as a glycan attached to EBOVAsn563 (Fig. 6d). A central interaction is formed by the heavy chain Tyr102 that intercalates between the Asn563-linked glycan and EBOV-Leu529 (Fig. 6d). Additional interactions include the burial of EBOV-Ile527 in a hydrophobic pocket, a hydrogen bond between EBOV-Trp531 and heavy chain His101, a hydrogen bond between EBOV-Gln521 and light chain Tyr92, as well as a close contact salt bridge and a hydrogen bond between EBOV-Glu564 and heavy chains Arg55 and Tyr54, respectively (Fig. 6d).

The epitope of 3T0331 on EBOV GP partially overlaps with the previously determined epitopes of ZMapp antibodies C4G7 and C2G4 (Fig. 6e) ${ }^{22,39,40}$. Notably, 3T0331 does not bind the side chain of Gln508 from EBOV GP2, which comprises an important part of the epitopes for other base-binding mAbs like $\mathrm{C} 2 \mathrm{G} 4{ }^{39}$. This residue is a known site for escape mutations ${ }^{41}$ and hence 3T0331 may tolerate substitutions at this position. Introducing a Q508R mutation, however, abrogated binding of 3T0331 (data not shown), suggesting that this alteration changes the main chain conformation in this region. Of note, the binding of $\mathrm{C} 2 \mathrm{G} 4$ requires some conformational changes at the N-terminal part of GP2 (Extended Data Fig. 9b), as opposed to 3T0331, which recognizes the native conformation (Extended Data Fig. 9c). Recognizing the native conformation rather than a presumably less stable altered conformation of EBOV GP may be energetically more favored, and may partially explain the higher potency of 3T0331.

Additionally, 3T0331 and 4m0368 are, in contrast to the chimeric antibodies C4G7 and C2G4, entirely of human origin, and the epitope of $4 \mathrm{~m} 0368$ overlaps with the EBOV GP-specific mAb100 $0^{23,42}$. Of note, when we assessed 3T0331 and 4m0368 for pharmacokinetic behavior in vivo as well as determining their autoreactivity in HepG2 cells, both antibodies showed no or only minor reactivity, and the pharmacokinetics were comparable to two control antibodies (HIV-1-specific antibody 3BNC117 and EBOV GP-specific antibody mAb114), which had half-lives of 17 days (3BNC117) and 24.2 days (mAb114), respectively, in clinical trials ${ }^{20,43}$ (Extended Data Fig. 10). In summary, these observations indicate that vaccine-elicited humoral immune responses effectively target previously identified vulnerability sites on EBOV GP and can serve as candidates for preventing and treating Ebolavirus infection.

\section{Discussion}

The demand for an Ebolavirus vaccine is increasing, and different potential vaccine candidates have been developed ${ }^{25,26,31}$. However, EVD vaccine trials aiming to study efficacy are often impeded by the outbreak nature of EVD and infrastructural challenges ${ }^{3,18}$. Therefore, it is of critical need to gain detailed mechanistic information on the induced antibody response to comprehensively inform on the development of the best possible EBOV vaccine strategies ${ }^{33,44}$.

Reproducibility is one of the hallmarks of effective vaccines. Among all of the closely examined subjects, we detected a convergent $B$ cell response that displays (1) the presence of shared antibody characteristics, (2) preferences of $\mathrm{V}$ genes found in EBOVreactive $m A b s$ and $(3)$ the frequent generation of $\mathrm{V}_{\mathrm{H}} 3-15 / \mathrm{V}_{\lambda} 1-40$ EBOV-neutralizing antibody class that showed traits of a recurrent affinity maturation process. Re-occurring antibodies that share the same $\mathrm{V}$ genes have occasionally been reported in response to infections with viruses such as HIV-1, Zika and West Nile virus ${ }^{45-47}$. For EVD, a longitudinal analysis of four survivors revealed a preference for IGHV3-13 in EBOV-neutralizing antibodies ${ }^{48}$. This gene is also utilized by $\mathrm{mAb} 114^{23}$ and by antibodies that were elicited upon DNA/Ad5 encoded EBOV GP immunization of macaques ${ }^{49}$. EBOV GP-neutralizing antibodies utilizing the same $\mathrm{V}_{\mathrm{H}}$ gene segment (IGHV3-13) were also detected in our analysis and showed shared antibody characteristics among immunized individuals. In addition, Rijal and colleagues recently isolated antibodies from human donors vaccinated with ChAd3-EBO-Z that were found to be neutralizing. Some of these harbored the combination of the IGHV315 and IGLV1-40 gene segments ${ }^{50}$, which we detected as a recurrent and EBOV GP-neutralizing antibody class in all vaccines studied.

As several species cause EVD in humans, antibodies that crossreact with additional species are of particular interest. Such crossreactive antibodies have been observed in EVD survivors ${ }^{14,15,51,52}$, and a recent study reported a cocktail of two pan-Ebolavirus antibodies for therapeutic protection of animal models ${ }^{10}$. Although crossreactivity was low in the sera of vaccinees, $30-75 \%$ of monoclonal antibodies potently cross-reacted with other Filoviruses. These data show that the rVSV-ZEBOV vaccine induces cross-reactive monoclonal antibodies and that the intensities of serum reactivities to Ebolavirus GPs alone are not always suitable to predict the development of such antibodies. Finally, epitope mapping revealed a broad coverage of epitopes of EBOV GP, which closely resembled antibodies previously isolated from EVD survivors ${ }^{14,15,36}$. These results indicate a successful translation of the antigenic profile from natural EBOV infection to the rVSV-ZEBOV vaccine.

EBOV-neutralizing activity has been demonstrated to be a critical factor for antibody-mediated protection ${ }^{26,27,36,53}$. Although little was known about the time of development of neutralizing antibodies, a recent longitudinal analysis of EVD survivors demonstrated a prolonged antibody maturation process and an increase in neutralizing activity, even many months after acute infection ${ }^{48}$. In another study, rVSV-ZEBOV vaccination was documented to induce an early IgM serum response and suggested a predominant role for IgM antibodies for neutralization and potentially for early protection ${ }^{54}$. In all analyzed rVSV-ZEBOV-vaccinated subjects, we discovered highly potent EBOV GP-specific IgG antibodies. The most potent neutralizers are competitors of the MPER-targeting ADI-15758 and ADI-15999 ${ }^{14}$, or of the

Fig. 5 | Recurrent development of an IGHV3-15/IGLV1-40 EBOV-neutralizing antibody class. a, Frequency of heavy chain (left) and light chain (right) $V$ genes in rVSV-ZEBOV-induced antibodies that neutralize EBOV Mayinga (dark blue), bind (light blue) or do not bind EBOV GP $\Delta T M$ (gray; $n=143$ ). Shown are $V$ genes found in at least one neutralizing antibody. Significant changes in $V$ gene frequency of neutralizing antibodies were tested using a binominal test under the null hypothesis of equal frequency, $\alpha=0.05$, and Bonferroni correction. Only IGHV3-15 and IGLV1-40 increases were highly significant with $P=2.2 \times 10^{-9}$. b. Frequency of neutralizing IGHV3-15-carrying antibodies. Slices represent antibodies of unrelated clones from individual donors (left) and corresponding light chains (right). c, Phylogenetic trees of IGHV3-15- (top) and IGLV1-40-expressing (bottom) neutralizing antibodies ( $n=17$ ). Pairwise distances were determined by multiple sequence alignment and Jukes-Cantor genetic distance model. Pairwise alignment scores (obtained by needle with default settings) of sequences from each donor were compared with scores from different donors. The difference of both scores was assessed by a twotailed Mann-Whitney-U test, with $P$ values of 0.409 (light) and 0.862 (heavy chains). d, Percent competition between IGHV3-15/IGLV1-40 antibodies for binding to EBOV GP $\triangle T M$ assessed by ELISA. Blue indicates competition and white shows simultaneous binding. Self-competition (in black contours) and competition with mAb114 were positive and ADI-15999 negative controls. e, Alignment of amino acid sequences of IGHV3-15- (top) and IGLV1-40expressing (bottom) neutralizing antibodies to germline sequence. Identical amino acids are indicated by dots, and amino acids at positions with $\geq 50 \%$ mutations are depicted in bold. Sequence Logo plots were generated by WebLogo 3. See also Extended Data Fig. 3. 
GP1/2-binding antibodies $\mathrm{KZ}^{2} 2^{35}$ and $\mathrm{mAb} 100^{23}$. Antibodies targeting these epitopes have been demonstrated to protect NHPs against Ebolavirus challenges ${ }^{23}$. Besides neutralizing activity, increasing attention has been paid to Fc-mediated effector functions that contribute to protection ${ }^{9,55-57}$. In our study, $51.7 \%$ of isolated EBOV-specific antibodies did not neutralize, and further studies a

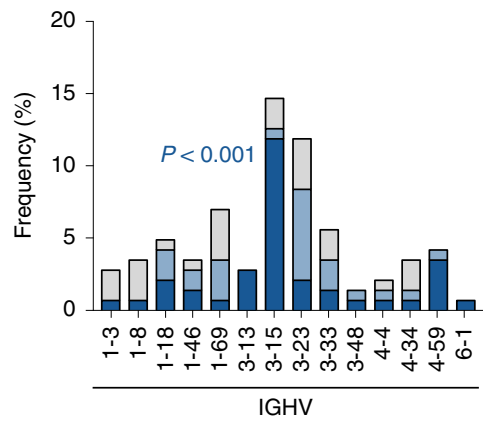

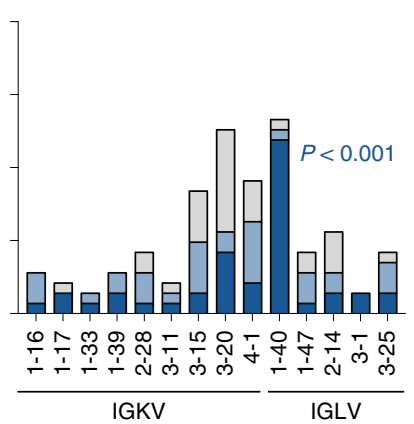

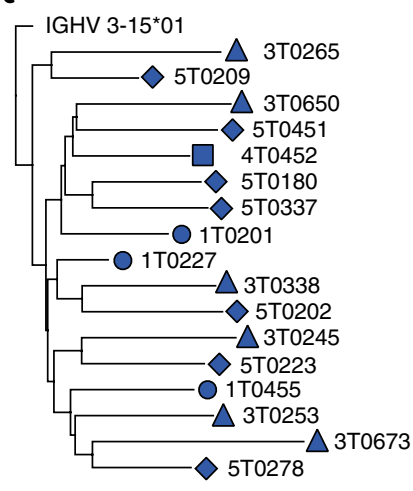

$\overline{0.025}$

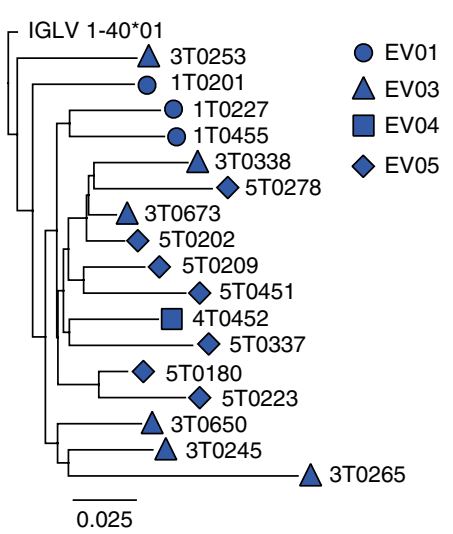

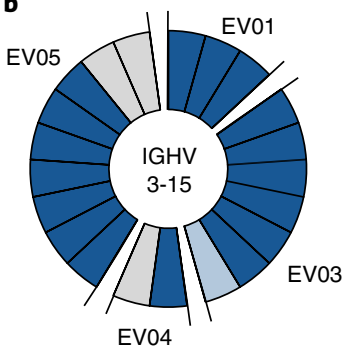

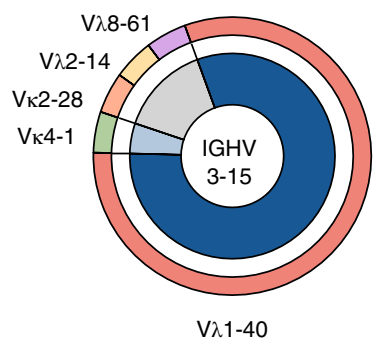

EBOV-neutralizing $\quad \square$ EBOV GP-binding $\quad \square$ Not binding

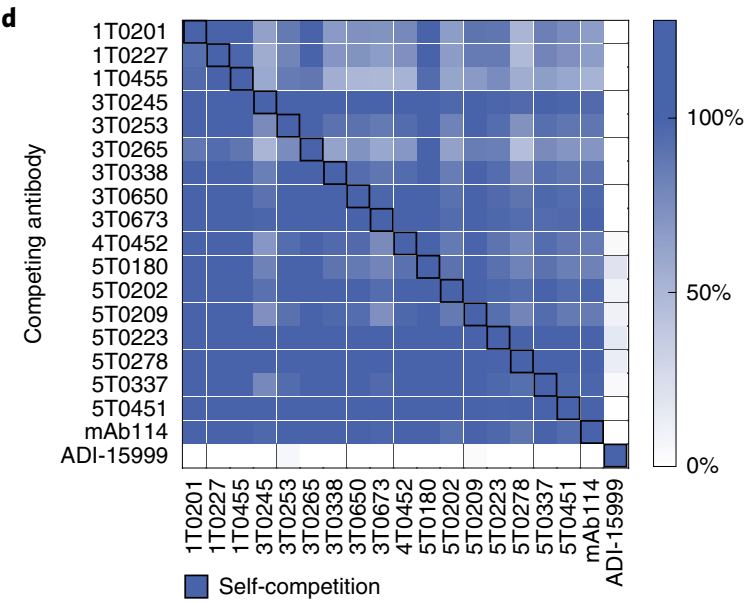

e
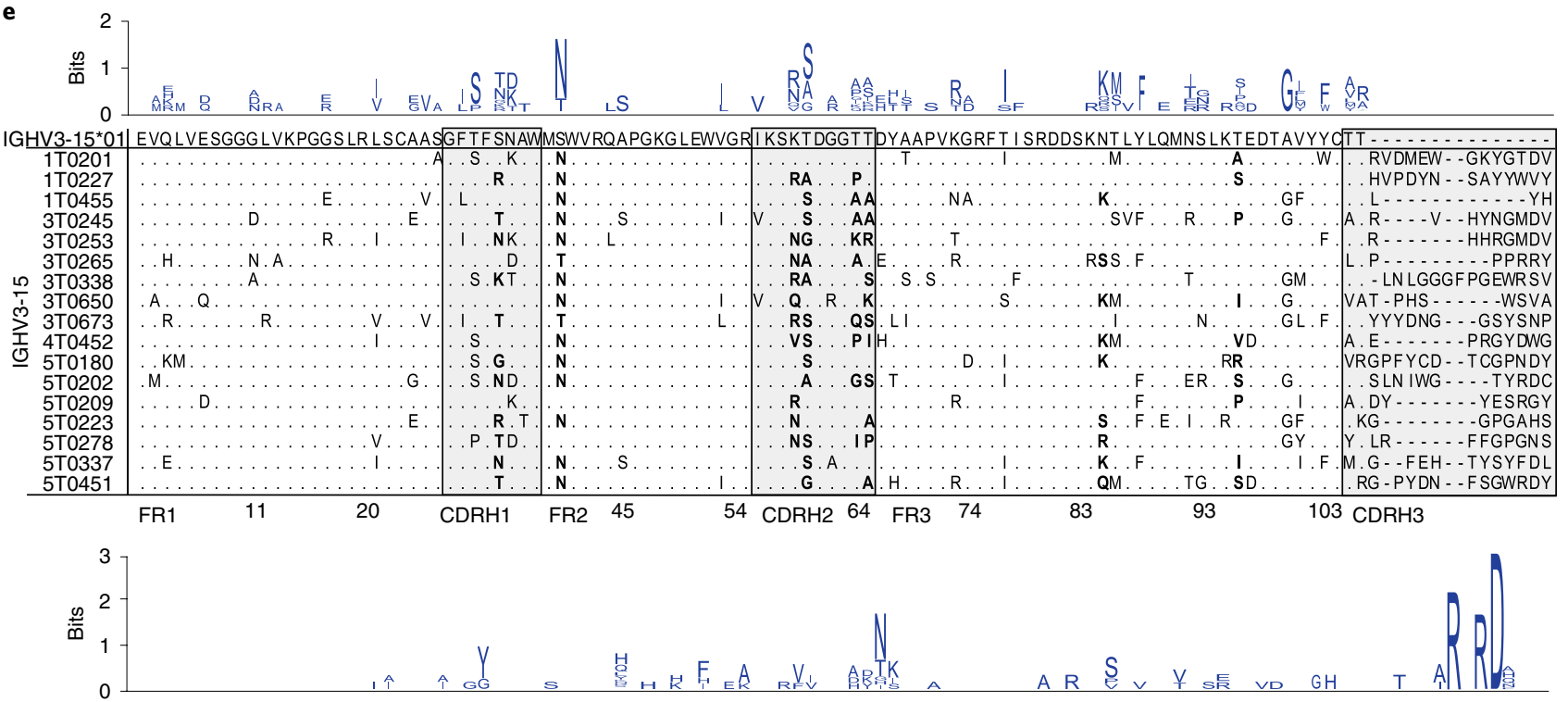

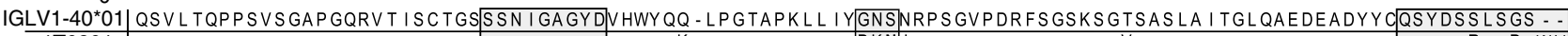

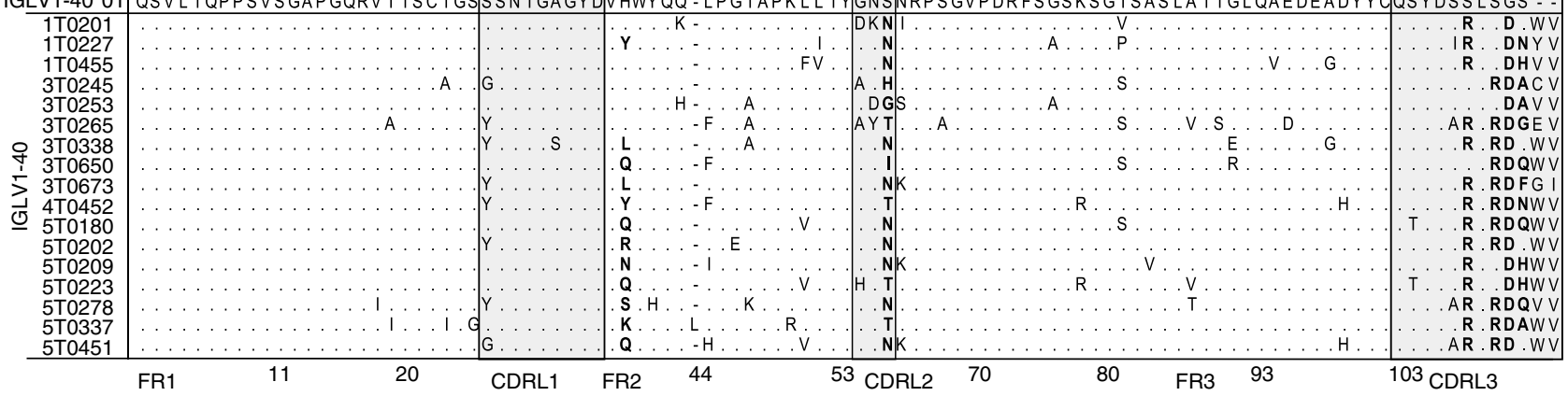


a

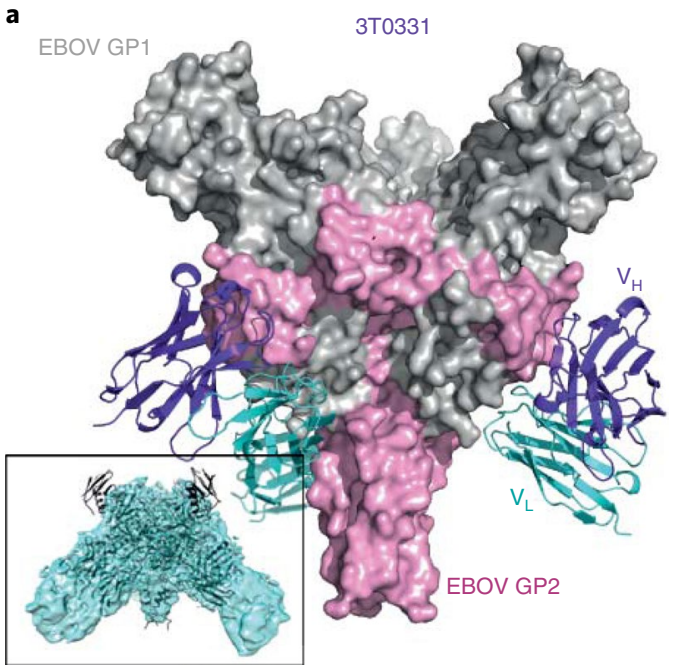

c

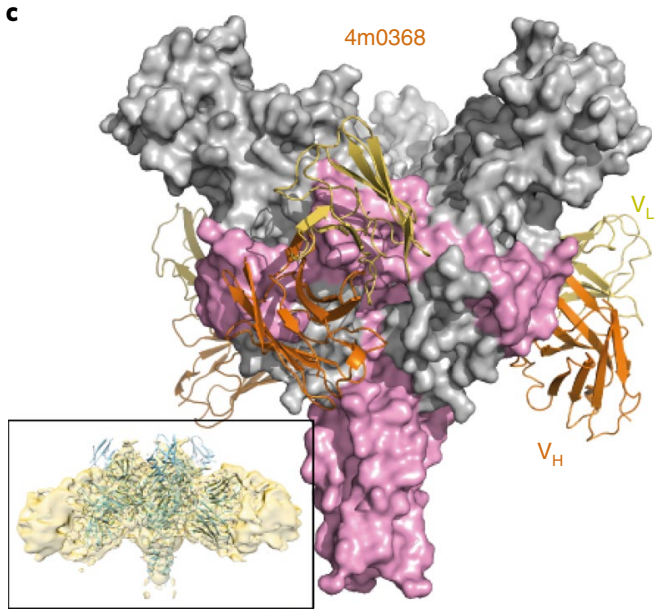

e

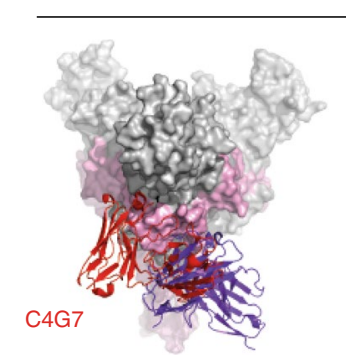

3Т0331

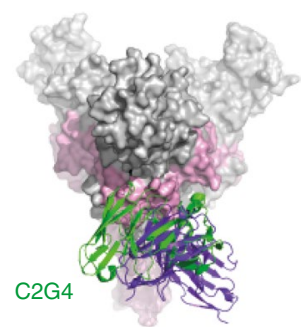

b

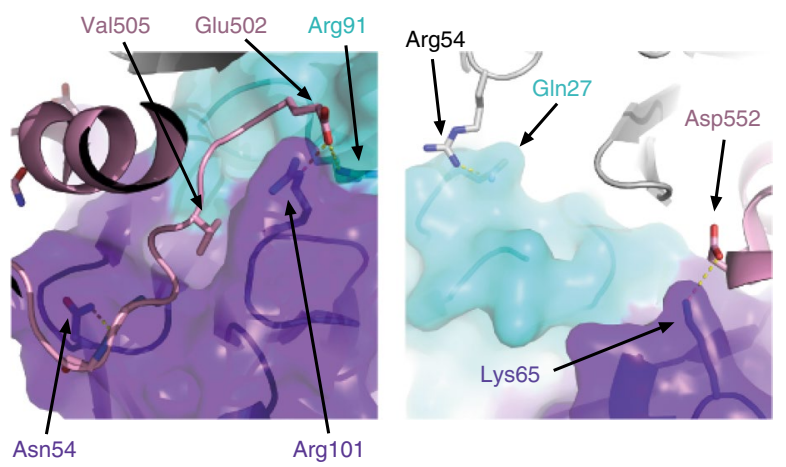

d

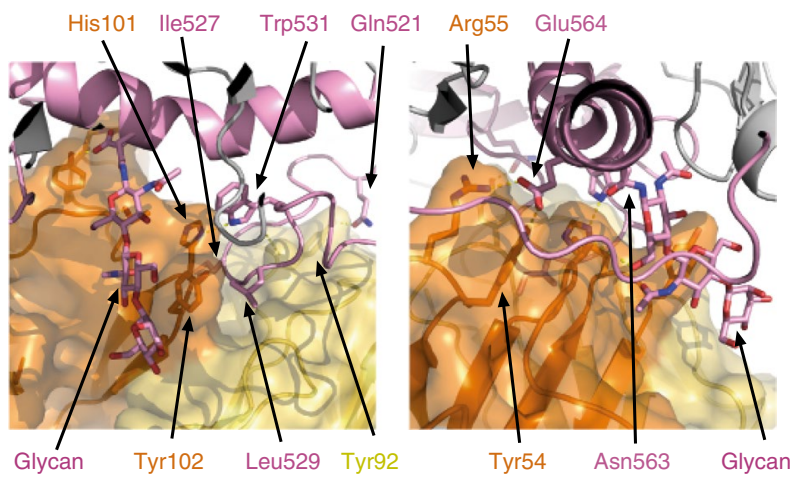

Fig. 6 | Neutralization determinants of mAbs $3 T 0331$ and $4 \mathrm{m0368}$. $a$, Structure of $3 T 0331-V_{H} V_{L}$, in a purple and cyan ribbon representation, bound to the trimeric EBOV GP, illustrated using a surface representation, with gray and pink for GP1 and GP2, respectively. Inset: The complete EBOV GP/3T0331 model in computed electron density (antibody in transparent cyan). b. Close-up views of the main contacts between 3 TO331 and EBOV GP using the same color scheme as in a. EBOV GP is shown in a ribbon representation and 3 T0331 is shown using a semi-transparent surface representation. The main polar interactions are illustrated with a yellow dashed line. c, Structure of $4 \mathrm{m0368}-\mathrm{V}_{\mathrm{H}} \mathrm{V}_{\mathrm{L}}$, shown as orange and yellow ribbons, bound to the trimeric EBOV GP, represented using the same color scheme as in a. Inset: The complete EBOV GP/4m0368 model in computed electron density (antibody in transparent yellow). d, Close-up views of the main contacts between $4 \mathrm{m0368}$ and EBOV GP, using the same color scheme as in c. e, Comparison of the EBOV GP recognitions sites of 3 T0331 (left) with therapeutic antibodies C2G7 (PDB 5KEN, left image, red), C2G4 (PDB 5KEL, middle image, green) and KZ52 (right image, turquoise) as well as comparison of $4 \mathrm{m0368}$ (right) to mAb100 (PDB 5FHC, magenta).

are needed to determine whether and how these rVSV-ZEBOVinduced antibodies may contribute to effective protection from EBOV infection.

A lack of humoral response to EVD infection is associated with fatal outcome in humans ${ }^{58}$, supporting the role of antibodies as treatment options against EVD. Today, antibodies are used as monotherapy (mAb114) or cocktails (ZMapp, REGN-EB3) to treat EVD under expanded access protocols and in clinical trials $^{18-22}$. Structural analyses of the potent neutralizer 3T0331 demonstrated a strong similarity with C4G7 and C2G4, both members of the ZMapp antibody cocktai ${ }^{22,41}$. Indeed, because of its favorable preclinical characteristics, including the highly potent neutralizing 
activity, and its fully human origin, 3T0331 is likely to be a promising candidate to use for clinical applications.

Finally, the identification of potent neutralizing rVSV-ZEBOVinduced antibodies demonstrates the great potential to obtain pathogen-directed human antibodies in the absence of infections and without access to convalescent survivors. VSV-based vaccines have been demonstrated to be generally safe and efficacious, and are currently being developed to protect individuals from other viruses, bacteria and parasites ${ }^{59}$. Although vaccine-induced antibodies have been isolated before ${ }^{50,60}$, our data highlight this opportunity for VSV-based vaccine strategies and therefore underline the potential to retrieve protecting antibodies that are of particular need in complicated outbreak scenarios and to combat emerging infections.

\section{Online content}

Any methods, additional references, Nature Research reporting summaries, source data, statements of code and data availability and associated accession codes are available at https://doi.org/10.1038/ s41591-019-0602-4.

Received: 3 May 2019; Accepted: 4 September 2019; Published online: 7 October 2019

\section{References}

1. WHO Ebola Haemorrhagic Fever http://www.who.int/mediacentre/factsheets/ fs103/en/ (2019).

2. WHO Ebola Situation Report 2016 http://apps.who.int/ebola/currentsituation/ebola-situation-report-30-march-2016 (2016).

3. Cohen, J. Worries about Ebola outbreak grow, despite use of vaccine. Science 362, 1225 (2018).

4. Maxmen, A. Ebola detectives race to identify hidden sources of infection as outbreak spreads. Nature 564, 174-175 (2018).

5. Towner, J. S. et al. Newly discovered Ebola virus associated with hemorrhagic fever outbreak in Uganda. PLoS Pathog. 4, e1000212 (2008).

6. Fields, B. N., Knipe, D. M. \& Howley, P. M. Fields Virology 5th edn (Wolters Kluwer Health/Lippincott Williams \& Wilkins, 2007).

7. Shears, P. \& O'Dempsey, T. J. Ebola virus disease in Africa: epidemiology and nosocomial transmission. J. Hosp. Infect. 90, 1-9 (2015).

8. Mbala-Kingebeni, P. et al. Rapid confirmation of the Zaire Ebola virus in the outbreak of the Equateur province in the Democratic Republic of Congo: implications for public health interventions. Clin. Infect. Dis. 68, 330-333 (2019).

9. Pascal, K. E. et al. Development of clinical-stage human monoclonal antibodies that treat advanced Ebola virus disease in nonhuman primates. J. Infect. Dis. 218, S612-S626 (2018).

10. Bornholdt, Z. A. et al. A two-antibody pan-Ebolavirus cocktail confers broad therapeutic protection in ferrets and nonhuman primates. Cell Host Microbe 25, 49-58.e45 (2019)

11. Murin, C. D. et al. Structures of protective antibodies reveal sites of vulnerability on Ebola virus. Proc. Natl Acad. Sci. USA 111, 17182-17187 (2014).

12. Qiu, X. et al. Reversion of advanced Ebola virus disease in nonhuman primates with ZMapp. Nature 514, 47-53 (2014)

13. Marzi, A. et al. Vaccines. An Ebola whole-virus vaccine is protective in nonhuman primates. Science 348, 439-442 (2015).

14. Bornholdt, Z. A. et al. Isolation of potent neutralizing antibodies from a survivor of the 2014 Ebola virus outbreak. Science 351, 1078-1083 (2016).

15. Flyak, A. I. et al. Cross-reactive and potent neutralizing antibody responses in human survivors of natural Ebolavirus infection. Cell 164, 392-405 (2016).

16. Flyak, A. I. et al. Broadly neutralizing antibodies from human survivors target a conserved site in the Ebola virus glycoprotein HR2-MPER region. Nat. Microbiol. 3, 670-677 (2018).

17. Warfield, K. L. et al. Role of antibodies in protection against Ebola virus in nonhuman primates immunized with three vaccine platforms. J. Infect. Dis. 218, S553-S564 (2018).

18. Maxmen, A. Experimental Ebola drugs face tough test in war zone. Nature 561, 14 (2018).

19. Damon, I. New tools in the Ebola arsenal. N. Engl. J. Med. 21, 1981-1982 (2018).

20. Gaudinski, M. R. et al. Safety, tolerability, pharmacokinetics, and immunogenicity of the therapeutic monoclonal antibody mAb114 targeting Ebola virus glycoprotein (VRC 608): an open-label phase 1 study. The Lancet 393, 889-898 (2019).

21. Sivapalasingam, S. et al. Safety, pharmacokinetics, and immunogenicity of a co-formulated cocktail of three human monoclonal antibodies targeting Ebola virus glycoprotein in healthy adults: a randomised, first-in-human phase 1 study. Lancet Infect. Dis. 18, 884-893 (2018).
22. Davey, R. T. Jr et al. A randomized, controlled trial of ZMapp for Ebola virus infection. N. Engl. J. Med. 375, 1448-1456 (2016).

23. Corti, D. et al. Protective monotherapy against lethal Ebola virus infection by a potently neutralizing antibody. Science 351, 1339-1342 (2016).

24. Kupferschmidt, K. Finally, some good news about Ebola: two new treatments dramatically lower the death rate in a trial. Science https:/doi.org/10.1126/ science.aaz1032 (2019).

25. Wong, G. et al. From bench to almost bedside: the long road to a licensed Ebola virus vaccine. Expert Opin. Biol. Ther. 18, 159-173 (2018).

26. Medaglini, D. \& Siegrist, C. A. Immunomonitoring of human responses to the rVSV-ZEBOV Ebola vaccine. Curr. Opin. Virol. 23, 88-94 (2017).

27. Henao-Restrepo, A. M. et al. Efficacy and effectiveness of an rVSV-vectored vaccine in preventing Ebola virus disease: final results from the Guinea ring vaccination, open-label, cluster-randomised trial (Ebola Ça Suffit!). Lancet 389, 505-518 (2017).

28. Ledgerwood, J. E. et al. Chimpanzee adenovirus vector Ebola vaccine. N. Engl. J. Med. 376, 928-938 (2017).

29. WHO. WHO Reaffirms Commitment to Democratic Republic of the Congo as Ebola Outbreak Nears 1000 Cases Amid Increased Violence https://www.who. int/news-room/detail/23-03-2019-who-reaffirms-commitment-to-thedemocratic-republic-of-the-congo-as-ebola-outbreak-nears-1-000-cases-amidincreased-violence (2019).

30. Wong, G. et al. Immunization with vesicular stomatitis virus vaccine expressing the Ebola glycoprotein provides sustained long-term protection in rodents. Vaccine 32, 5722-5729 (2014).

31. Suder, E., Furuyama, W., Feldmann, H., Marzi, A. \& de Wit, E. The vesicular stomatitis virus-based Ebola virus vaccine: from concept to clinical trials. Hum. Vaccin. Immunother. 14, 2107-2113 (2018).

32. WHO Preliminary Results on the Efficacy of rVSV-ZEBOV-GP Ebola Vaccine Using the Ring Vaccination Strategy in the Control of an Ebola Outbreak in the Democratic Republic of the Congo: An Example of Integration of Research into Epidemic Response https:/www.who.int/csr/resources/publications/ebola/ ebola-ring-vaccination-results-12-april-2019.pdf (2019).

33. WHO Ebola Vaccines - Background Paper for SAGE (Strategic Advisory Group of Experts) Deliberations https://www.who.int/immunization/sage/ meetings/2018/october/2_Ebola_SAGE2018Oct_BgDoc_20180919.pdf (2018).

34. WHO Compassionate Use of Ebola Vaccine in the Context of the Ebola Outbreak in North Kivu, Democratic Republic of the Congo https://www.who. int/emergencies/diseases/ebola/frequently-asked-questions/ebola-vaccine (2018)

35. Lee, J. E. et al. Structure of the Ebola virus glycoprotein bound to an antibody from a human survivor. Nature 454, 177-182 (2008).

36. Saphire, E. O. et al. Systematic analysis of monoclonal antibodies against Ebola virus GP defines features that contribute to protection. Cell 174, 938-952 e913 (2018).

37. Zhao, Y. et al. Toremifene interacts with and destabilizes the Ebola virus glycoprotein. Nature 535, 169-172 (2016).

38. Norn, C. H., Lapidoth, G. \& Fleishman, S. J. High-accuracy modeling of antibody structures by a search for minimum-energy recombination of backbone fragments. Proteins 85, 30-38 (2017).

39. Pallesen, J. et al. Structures of Ebola virus GP and sGP in complex with therapeutic antibodies. Nat. Microbiol. 1, 16128 (2016).

40. Qiu, X. et al. Characterization of Zaire Ebolavirus glycoprotein-specific monoclonal antibodies. Clin. Immunol. 141, 218-227 (2011).

41. Qiu, X. et al. Successful treatment of Ebola virus-infected cynomolgus macaques with monoclonal antibodies. Sci. Transl. Med. 4, 138ra181 (2012).

42. Misasi, J. et al. Structural and molecular basis for Ebola virus neutralization by protective human antibodies. Science 351, 1343-1346 (2016).

43. Caskey, M. et al. Viraemia suppressed in HIV-1-infected humans by broadly neutralizing antibody 3BNC117. Nature 522, 487-491 (2015).

44. Davey, R. T. et al. The past need not be prologue: recommendations for testing and positioning the most-promising medical countermeasures for the next outbreak of Ebola virus infection. J. Infect. Dis. 218, S690-S697 (2018).

45. Setliff, I. et al. Multi-donor longitudinal antibody repertoire sequencing reveals the existence of public antibody clonotypes in HIV-1 infection. Cell Host Microbe 23, 845-854 e846 (2018).

46. Robbiani, D. F. et al. Recurrent potent human neutralizing antibodies to Zika virus in Brazil and Mexico. Cell 169, 597-609.e511 (2017).

47. Throsby, M. et al. Isolation and characterization of human monoclonal antibodies from individuals infected with West Nile virus. J. Virol. 80, 6982-6992 (2006).

48. Davis, C. W. et al. Longitudinal analysis of the human B cell response to ebola virus infection. Cell 177, 1566-1582.e17 (2019).

49. Cagigi, A. et al. Vaccine generation of protective Ebola antibodies and identification of conserved B-cell signatures. J. Infect. Dis. 218, S528-S536 (2018).

50. Rijal, P. et al. Therapeutic monoclonal antibodies for Ebola virus infection derived from vaccinated humans. Cell Rep. 27, 172-186.e177 (2019). 
51. Bramble, M. S. et al. Pan-filovirus serum neutralizing antibodies in a subset of Congolese Ebolavirus infection survivors. J. Infect. Dis. 218, 1929-1936 (2018).

52. King, L. B., Milligan, J. C., West, B. R., Schendel, S. L. \& Ollmann Saphire, E. Achieving cross-reactivity with pan-Ebolavirus antibodies. Curr. Opin. Virol. 34, 140-148 (2019).

53. Marzi, A. et al. Antibodies are necessary for rVSV/ZEBOV-GP-mediated protection against lethal Ebola virus challenge in nonhuman primates. Proc. Natl Acad. Sci. USA 110, 1893-1898 (2013).

54. Khurana, S. et al. Human antibody repertoire after VSV-Ebola vaccination identifies novel targets and virus-neutralizing IgM antibodies. Nat. Med. 22 1439-1447 (2016).

55. Saphire, E. O., Schendel, S. L., Gunn, B. M., Milligan, J. C. \& Alter, G. Antibody-mediated protection against Ebola virus. Nat. Immunol. 19, 1169-1178 (2018).

56. Gunn, B. M. et al. A role for Fc function in therapeutic monoclonal antibody-mediated protection against Ebola virus. Cell Host Microbe 24, 221-233.e225 (2018).

57. Liu, Q. et al. Antibody-dependent-cellular-cytotoxicity-inducing antibodies significantly affect the post-exposure treatment of Ebola virus infection. Sci. Rep. 7, 45552 (2017).

58. Baize, S. et al. Defective humoral responses and extensive intravascular apoptosis are associated with fatal outcome in Ebola virus-infected patients. Nat. Med. 5, 423-426 (1999).

59. Clarke, D. K. et al. Live virus vaccines based on a vesicular stomatitis virus (VSV) backbone: standardized template with key considerations for a risk/ benefit assessment. Vaccine 34, 6597-6609 (2016).

60. Cagigi, A. et al. Vaccine-mediated induction of an Ebolavirus cross-species antibody binding to conserved epitopes on the glycoprotein heptad repeat 2/ membrane-proximal external junction. J. Infect. Dis. 218, S537-S544 (2018).

\section{Acknowledgements}

We thank all study participants who devoted time to our research. We thank all members of the Klein, Becker, Diskin and Pfeifer Laboratories for helpful discussion; D. Weiland and S. Borregaard for help with recruitment and study implementation; C. Scheid and U. Holtig for supervising leukapheresis procedures of enrolled study participants; $\mathrm{H}$. Janicki, K. Jain and C. Ruping for help with sample processing, antibody cloning and antibody production; the Cologne Center for Genomics (CCG) for sequencing support; M. Carroll for providing sera of EVD survivors and S. Fehling and T. Strecker for analysis of the neutralizing activity of sera from EVD survivors. Diffraction experiments were performed at beamline ID23-2 at the European Synchrotron Radiation Facility (ESRF), Grenoble, France. We are grateful to S. Hutin at the ESRF for providing assistance in using beamline ID23-2. Electron microscopy studies were supported in part by the Irving and Cherna Moskowitz Center for Nano and Bio-Nano Imaging at the Weizmann Institute of Science. Coordinate files, structure factors and density maps of 3T0331Fab, EBOV-GP/3T0331 and EBOV-GP/4m0368 were deposited and are available at the Protein Data Bank under accession codes 6QCU, 6QD7 and 6QD8, respectively. This work was funded by grants from the German Center for Infection Research (DZIF, M.M.A., S.B. and F.K.), the German Research Foundation (CRC 1279, F.K.; CRC 1310, F.K.; Heisenberg-Program KL2389/2-1, F.K.), the European Research Council (ERC-StG639961, F.K.) and the DFG Cluster of Excellence 'Machine Learning-New Perspectives for Science' (EXC 2064/1, project no. 390727645, N.P.).

\section{Author contributions}

Conceptualization was provided by F.K., methodology by F.K., R.D., S.B., C.K. and N.P., investigations by S.A.E., M.Z., V.K., H.C.-D., C.K., N.E., H.G., L.G. and M.S.E., software by C.K., formal analysis by S.A.E., M.Z., C.K., H.C.-D., V.K., R.E., N.P., R.D. and F.K., and resources by F.K., S.B., M.M.A., C.D., P.S. and T.W. The original draft of the manuscript was written by F.K., M.Z., S.A.E., R.D. and C.K. and further writing, review and editing by F.K., S.A.E., M.Z., C.K., R.D., S.B., V.K., H.G., N.P. and R.E. The project was supervised by F.K

\section{Competing interests}

The antibodies reported in the article and the approach for their identification are in the process of being patented.

\section{Additional information}

Extended data is available for this paper at https://doi.org/10.1038/s41591-019-0602-4. Supplementary information is available for this paper at https://doi.org/10.1038/ s41591-019-0602-4.

Correspondence and requests for materials should be addressed to F.K.

Peer review information Alison Farrell is the primary editor on this article and managed its editorial process and peer review in collaboration with the rest of the editorial team.

Reprints and permissions information is available at www.nature.com/reprints.

Publisher's note Springer Nature remains neutral with regard to jurisdictional claims in published maps and institutional affiliations.

(c) The Author(s), under exclusive licence to Springer Nature America, Inc. 2019 


\section{Methods}

All information regarding material and methods can also be found in the Life Sciences Reporting Summary.

rVSV-ZEBOV-GP-vaccinated individuals and sample collection. Individuals participating in this study were previously enrolled in the Phase I Trial to Assess the Safety, Tolerability and Immunogenicity of an Ebola Virus Vaccine (rVSV $\Delta \mathrm{G}$ ZEBOV-GP; NCT02283099) and were vaccinated with either $3 \times 10^{5}$ or $3 \times 10^{6}$ p.f.u. From this cohort, six individuals were enrolled in an observational study (INA; $16-$ 054) at the University Hospital of Cologne to collect serum and PBMC samples. An additional individual vaccinated with $2 \times 10^{7}$ p.f.u. was also included. The protocol was approved by the Institutional Review Board (IRB) of the University of Cologne, Germany. From all participants, serum, EDTA blood and/or leukapheresis samples were collected and PBMCs were purified by density gradien centrifugation using HistoPaque and stored in FBS containing 10\% DMSO (all Merck) at $-150^{\circ} \mathrm{C}$. Serum and plasma samples were stored at $-80^{\circ} \mathrm{C}$. All enrolled subjects provided written informed consent before participation in the study and all aspects of study conduct were in accordance with Good Clinical Practice. Sera of Ebola virus survivors were collected in the context of the project EVIDENT (Ebola Virus Disease: Correlates of Protection, Determinants of Outcome and Clinical Management) after obtaining informed consent and provided to use as control samples for anti-EBOV IgG titer and neutralizing activity. PBMCs and serum samples from the individuals studied are limited and can only be provided in limited quantities on request.

Expression and purification of Ebolavirus GPs. Recombinant Ebolavirus GP constructs lacking the TM domain ( $\Delta 651-676$; EBOV Makona (GenBank KJ660347), EBOV Mayinga (GenBank AF086833.2), BDBV (GenBank FJ217161), SUDV Gulu (GenBank AY729654.1) or TM and MLD domain ( $\Delta 313-464$; EBOV Makona, GenBank KJ660347) as well as sGP (GenBank KJ660347) were cloned into the pCAGGS backbone ${ }^{61}$. GP constructs were further modified to carry an Avi-tag as well as a His-tag for purification, and, except sGP, a GCN4 domain for GP complex formation at the C-terminal end ${ }^{23}$. HEK293F cells were maintained in FreeStyle Medium (Life Technologies) at $37^{\circ} \mathrm{C}$ with $6 \% \mathrm{CO}_{2}$, shaking at 110 r.p.m. At a concentration of $0.8 \times 10^{6} \mathrm{cells}^{-1}$, transfection was performed using $25 \mathrm{kDa}$ polyethylenimine (PEI, Polysciences) with $1 \mu \mathrm{g} \mathrm{DNA} \mathrm{ml}{ }^{-1}$ cell suspension. After incubating for seven days, the supernatant was harvested, filtered $(0.45 \mu \mathrm{m}$ filter; Nalgene, Thermo Fisher Scientific) and incubated with Protino Ni-NTA (nitrilotriacetic acid) agarose beads (Macherey Nagel) overnight. Beads were pelleted, transferred into a column and washed once with NPI-10 $\left(\mathrm{H}_{2} \mathrm{O}, 50 \mathrm{mM}\right.$ $\mathrm{NaH}_{2} \mathrm{PO}_{4}, 300 \mathrm{mM} \mathrm{NaCl}, 10 \mathrm{mM}$ imidazole, $\mathrm{pH}$ 8) and once with NPI- $20\left(\mathrm{H}_{2} \mathrm{O}\right.$, $50 \mathrm{mM} \mathrm{NaH}_{2} \mathrm{PO}_{4}, 300 \mathrm{mM} \mathrm{NaCl}, 20 \mathrm{mM}$ imidazole, $\mathrm{pH}$ 8) buffer. His-tagged glycoproteins were eluted by addition of NPI-100 $\left(\mathrm{H}_{2} \mathrm{O}, 50 \mathrm{mM} \mathrm{NaH} \mathrm{PO}_{4}, 300 \mathrm{mM}\right.$ $\mathrm{NaCl}, 100 \mathrm{mM}$ imidazole, $\mathrm{pH} 8$ ) and buffer was exchanged to PBS using centrifugal filter units (Amicon MWCO $30 \mathrm{kDa}$ for GP $\Delta$ MLD $\Delta \mathrm{TM}$ and GP $\Delta \mathrm{TM}$ or $10 \mathrm{kDa}$ for sGP; Merck). All glycoproteins were filtered $\left(0.22 \mu \mathrm{m}\right.$; Merck) and stored at $-80^{\circ} \mathrm{C}$.

Cell surface expression of anti-EBOV GP-specific antibodies to validate close resemblance of produced EBOV GP $\Delta$ TM to the native conformation. Variable regions of human EBOV GP-specific antibodies $\mathrm{KZ}^{2} 2^{62}, \mathrm{mAb} 114, \mathrm{mAb} 100^{23}$, ADI-15758, ADI-15999 and ADI-16037 ${ }^{14}$ were cloned into pMX vector with heavy and light chain constant regions ${ }^{63}$ using Gibson Assembly Master Mix (NEB). Constructs carried self-splicing signal F2A, PDGF-R transmembrane domain and mCherry reporter protein. HEK293T cells were transfected with constructs performing the $\mathrm{CaCl}_{2}$ protocol. Cells were harvested two days post transfection, suspended in PBS (Gibco) with 2\% FBS (Merck) and 2 mM EDTA (Thermo Fisher Scientific) and subjected to fluorescence staining with DAPI (Thermo Fisher Scientific), anti-huIgG-APC (BD) and EBOV GP $\Delta$ TM labeled with DyLight 488 (Microscale Antibody Kit, Thermo Fisher Scientific). Cells were stained for $20 \mathrm{~min}$ at $4{ }^{\circ} \mathrm{C}$, washed, and binding of GP $\Delta$ TM to anti-EBOV GP-specific cells was assessed by flow cytometry with FACSAria III (Becton Dickinson).

Single cell sort of EBOV GPATM-specific IgG $^{+}$B cells. PBMCs were enriched for B lymphocytes by magnetic cell separation (MACS) with CD19 microbeads (Miltenyi Biotec) according to the manufacturer's instructions. MACS LS separation columns (Miltenyi Biotec) were used to enrich labeled cells. B cells were spun and suspended in PBS (Gibco) with $2 \%$ FBS (Merck) and 2 mM EDTA (Thermo Fisher Scientific) and subjected to fluorescence staining with DAPI (Thermo Fisher Scientific), anti-huCD20-Alexa Fluor 700 (BD), anti-huIgG-APC (BD) and EBOV GP $\triangle$ TM-DyLight 488 (Microscale Antibody Kit, Thermo Fisher Scientific). Cells were stained for $20 \mathrm{~min}$ at $4{ }^{\circ} \mathrm{C}$, and live $\mathrm{CD} 20^{+}, \mathrm{IgG}^{+}$and $\mathrm{EBOV}$ $\mathrm{GP}^{+}$cells were sorted using a FACSAriaIII (Becton Dickinson) in a single cell manner into 96 -well plates. All wells contained $4 \mu \mathrm{l}$ lysis buffer $\left(0.5 \times \mathrm{PBS}, 0.5{\mathrm{U} \mathrm{l}^{-1}}^{-1}\right.$ RNAsin (Promega), $0.5 \mathrm{U} \mathrm{ul}^{-1}$ RNaseOUT (Thermo Fisher Scientific) and $10 \mathrm{mM}$ dithiothreitol (Thermo Fisher Scientific)). After sorting, plates were immediately stored at $-80^{\circ} \mathrm{C}$ until further processing.

Ig heavy/light chain amplification and sequence analysis. The procedure for PCR amplification was performed as previously described ${ }^{64,65}$ but with various adjustments. Briefly, random hexamers (Invitrogen), NP-40 and RNAseOUT (Thermo Fisher Scientific) were added to sorted cells in lysis buffer. The reaction was incubated for $1 \mathrm{~min}$ at $65^{\circ} \mathrm{C}$ and cooled for at least $2 \mathrm{~min}$ on ice. Afterwards, $1 \times$ Superscript IV RT buffer, $50 \mathrm{U} / \mathrm{rxn}$ SuperScript IV reverse transcriptase, dNTPs, dithiothreitol, RNAseOUT (all Thermo Fisher Scientific) and RNasin (Promega) were added and incubated $15 \mathrm{~min}$ at room temperature, $10 \mathrm{~min}$ at $50^{\circ} \mathrm{C}$ and $10 \mathrm{~min}$ at $80^{\circ} \mathrm{C}$. cDNA was used to amplify light and heavy chains using PlatinumTaq HotStart polymerase (Thermo Fisher Scientific) according to the manufacturer's protocol with $6 \% \mathrm{~KB}$ extender and $4 \mu \mathrm{l} \mathrm{cDNA}$ template. Amplification was achieved by sequential semi-nested PCRs using optimized and newly developed primer sets (Kreer et al., submitted). The results of the PCRs were analyzed by gel electrophoresis and products of correct sizes were subjected to Sanger sequencing.

Sequencing chromatograms were filtered for a mean Phred score of 28 and a minimal length of $240 \mathrm{nt}$. Remaining sequences were annotated with IgBLAST $^{66}$ and trimmed to extract only the variable region from FWR1 to the end of the J gene. Base calls within the variable region with a Phred score below 16 were masked and sequences with more than 15 masked nucleotides, stop codons or frameshifts were excluded from further analyses. To identify clonally related sequences within a single subject, we grouped all productive heavy chain sequences of that particular subject by identical $\mathrm{V}_{\mathrm{H}}$ genes, determined the pairwise Levenshtein distance for their CDRH3s, and assigned an individual clone number to sequence groups that share the same $\mathrm{V}_{\mathrm{H}}$ gene and have a minimal CDRH3 identity of $75 \%$ (with respect to the shortest CDRH3). A total of 100 rounds of input sequence randomization and clonal assignment were performed, and the result with the lowest number of remaining unassigned (non-clonal) sequences was selected for downstream analyses. All clones were cross-validated by the investigators, also taking into account shared mutations. Sequences were denoted as 'shared sequences' across vaccinees if two heavy chains from two different donors had the same $\mathrm{V}_{\mathrm{H}}$ gene and at least 75\% CDRH3 identity (as determined by the Levenshtein distance divided by the length of the shorter CDR3Hs). All members from an intra-donor clone were assigned to a 'shared sequence pool' if the heavy chain of at least one clonal member was identified as a shared sequence. For the identification of the closest sequence pairs between different donors, pairwise Levenshtein distances were calculated for sequences with identical $\mathrm{V}_{\mathrm{H}}$ genes from different donors and normalized by the shorter $\mathrm{V}_{\mathrm{H}}$ gene length. CDRH3 distances for the most similar pairs were determined the same way and in the case of two matches with the same overall $\mathrm{V}_{\mathrm{H}}$ gene similarity, preference was given to the pair with the most similar CDRH3s.

Cloning and production of EBOV GP-specific mAbs. Selected antibody sequences were cloned into $\mathrm{mAb}$ expression vectors by sequence- and ligationindependent cloning (SLIC) as previously described ${ }^{65}$. Briefly, amplicons for cloning were produced by PCR amplification using Q5 High Fidelity polymerase (NEB) by standard PCR protocols and previously described primers ${ }^{64}$. PCR products were purified (GeneJET Gel Extraction and DNA Cleanup Micro Kit, Macherey Nagel), cloned into expression vectors by SLIC reaction using T4 DNA polymerase (NEB) and transduced into Escherichia coli DH5 $\alpha$. In total, 4-8 colonies were evaluated by PCR and gel electrophoresis, and forwarded to Sanger sequencing. Plasmids with correct antibody sequences were amplified by midi preparation (Macherey Nagel kit) according to the manufacturer's protocol and stored at $4{ }^{\circ} \mathrm{C}$ (short term) or $-20^{\circ} \mathrm{C}$.

Heavy and light chain-encoding plasmids were co-transfected into HEK293E cells with PEI reagent as described above. Cultures were harvested after seven days, cell-free supernatants were filtered $(0.45 \mu \mathrm{m}$ filter; Nalgene, Thermo Fisher Scientific) and then incubated with Protein $\mathrm{G}$ sepharose (Merck) at $4{ }^{\circ} \mathrm{C}$ overnight. Beads were transferred to columns, washed three times with PBS and antibodies were eluted with $0.1 \mathrm{M}$ Glycine $(\mathrm{pH} 3)$. Subsequently, the $\mathrm{pH}$ of the eluate was neutralized with $1 \mathrm{M}$ Tris- $\mathrm{HCl}(\mathrm{pH} 8)$. Buffer was exchanged to PBS as described above (in 'Expression and purification of Ebolavirus GPs').

ELISA analysis to determine antibody binding activity to GPs. ELISAs were conducted in high binding 96-well ELISA plates (Corning). Plates were coated with $2.5 \mu \mathrm{g} \mathrm{ml}^{-1} \mathrm{GPs}$ at $4{ }^{\circ} \mathrm{C}$ overnight, washed three times with PBST (PBS, $0.05 \%$ Tween-20) and blocked with PBST/2\% BSA (Carl Roth) for $60 \mathrm{~min}$ at room temperature. mAbs were tested at four- or six-fold dilutions (1:3 or 1:5) with starting concentrations of $10 \mu \mathrm{g} \mathrm{ml}^{-1}$. After incubation for $90 \mathrm{~min}$ at room temperature, plates were washed three times and incubated with horseradish peroxidase (HRP)conjugated goat anti-human IgG antibody (Jackson ImmunoResearch, 1:2,500 in PBST/2\% BSA) for 60 min at RT. Reaction was started with 2,2' -azino-bis(3ethyl-benzothiazoline-6-sulfonic acid) solution (ABTS, Thermo Fisher Scientific) and the absorbance (optical density (OD) at 415-695 nm) was measured with an absorbance reader (Tecan) after fixed incubation times. For analysis, time points with overlapping standards curves were selected and ODs as well as EC $_{50}$ were determined. Antibodies were considered GP-reactive if the OD was above 0.2 at a $\mathrm{mAb}$ concentration of $10 \mu \mathrm{g} \mathrm{ml}^{-1}$ and the $\mathrm{EC}_{50}$ did not exceed $15 \mu \mathrm{g} \mathrm{ml}^{-1}$.

EBOV GP $\Delta$ TM peptide library. EBOV GP $\Delta$ TM strain Makona was divided into 80 peptides with a length of 18 aa and an overlap of 10 aa. Peptides were synthesized by Thermo Fisher Scientific PEPOTEC IMMUNO (project 
BC100718.1) with an $\mathrm{N}$-terminal acetylation and a C-terminal amidation. Peptides were dissolved in PBS, DMSO (Merck) or dimethyl formamide (DMF, Merck), depending on their sequence characteristics. Peptides were stored at $5 \mathrm{mg} \mathrm{ml}^{-1}$ and diluted in PBS for ELISA plate coating $\left(10 \mu \mathrm{g} \mathrm{ml}^{-1}\right)$. ELISA was performed as described above (in 'ELISA analysis to determine antibody binding activity to GPs') with an antibody concentration of $30 \mu \mathrm{g} \mathrm{ml}^{-1}$.

EBOV GP competition ELISA. EBOV GP-specific antibodies were added to EBOV GP $\triangle$ TM-coated ELISA plates for 90 min under different concentrations. After washing, biotin-labeled (EZ-Link sulfo-NHS-biotin, Thermo Fisher Scientific) competition mAbs with known epitopes (ADI-15758, ADI-15999 ${ }^{14}$, $\mathrm{KZ}_{2} 2^{35}$ and $\left.\mathrm{mAb} 100^{23}\right)$ were added for $45 \mathrm{~min}$ in PBST/2\% BSA $\left(0.5 \mu \mathrm{g} \mathrm{ml}^{-1}\right)$. For detection, HRP-conjugated streptavidin (1:8,000; Thermo Fisher Scientific) in combination with ABTS reagent was used. Full inhibition was determined by self-competition of the tested competition antibody. Positive antibody competition was defined as at least $25 \%$ of detected signal reduction during self-competition.

Epitope determination of antibodies. The epitopes of EBOV GP-reactive antibodies were determined by combining the ELISA results using EBOV Makona GP $\Delta$ TM $\Delta$ MLD and sGP with competition analysis as well as peptide library. sGP binding indicates an epitope within the N-terminal part of GP1 that is shared between GP and sGP. Missing interaction with EBOV GP $\Delta$ TM $\Delta$ MLD and sGP was interpreted as major interaction with the MLD. Positive competition with ADI-15758 or ADI-15999 shows an epitope at the C-terminal end of GP2, and competition with KZ52 or mAb100 was defined as an epitope at the interface or base of GP1/GP2. For refinement of the ELISA results and identification of additional antibody epitopes, peptide library results were used.

HepG2 cell assay to determine autoreactivity. HepG2 cells (NOVA Lite HEp-2 ANA Kit, Inova Diagnostics) were stained with $100 \mu \mathrm{g} \mathrm{ml}^{-1} \mathrm{mAb}$ for $30 \mathrm{~min}$ at room temperature followed by washing with PBS and labeling with a second FITCconjugated anti-human IgG antibody for $30 \mathrm{~min}$. Stained slides were mounted and analyzed in a wide-field fluorescence microscope (Leica DMI microscope) and fluorescence intensity was evaluated in a blinded way by four independent persons. Each $\mathrm{mAb}$ was tested at least in duplicate and staining was categorized into a scoring system ranging from 0 to 3 .

Neutralization assays. Sera or antibodies (starting at a dilution of 1:8 (sera) or a concentration of $100 \mathrm{~g} \mathrm{~m} \mathrm{~m}^{-1}$ (antibodies)) were serially diluted in 96-well culture plates in Dulbecco's modified Eagle's medium (DMEM, Thermo Fisher Scientific) supplemented with $2 \%$ fetal calf serum, penicillin $\left(50 \mathrm{U} \mathrm{ml}^{-1}\right)$, streptomycin $\left(50 \mu \mathrm{g} \mathrm{ml}^{-1}\right)$ and L-glutamine $(2 \mathrm{mM}) .100 \mathrm{TCID}_{50}$ units of EBOV Mayinga (GenBank NC_002549) and SUDV Boniface (GenBank FJ968794.1) were added to the serum or antibody dilutions. Following incubation at $37^{\circ} \mathrm{C}$ for $1 \mathrm{~h}$, Vero C1008 cells (ATCC CRL-1586; 10,000 cells per well) were added. Plates were then incubated at $37^{\circ} \mathrm{C}$ with $5 \% \mathrm{CO}_{2}$, and cytopathic effects (CPE) were evaluated at day 7 post infection. Neutralization was defined as clear reduction of CPE in serum or antibody dilutions compared to positive controls. Neutralization titers of four replicates were calculated as geometric mean titers for sera (reciprocal value) or geometric mean concentrations in the case of monoclonal antibodies. The cutoff of the assay was determined by the first dilution of the respective serum or antibody. Neutralization assays were performed in the BSL-4 laboratory of the Institute of Virology, Philipps-University Marburg, Germany.

Unbiased B cell repertoire analyses. B cell receptor repertoire sequence data were generated by an unbiased template-switch-based NGS approach. In brief, 250,000 $\mathrm{CD}^{2} 0^{+} / \mathrm{IgG}^{+} \mathrm{B}$ cells were sorted for each individual and RNA was isolated with the RNeasy Micro Kit (Qiagen) using a QiaCube (Qiagen) instrument. cDNA was generated by template-switch reverse transcription according to the SMARTer RACE 5'/3' manual (Takara/Clonetech) with a self-designed template-switch oligo that included an $18 \mathrm{nt}$ unique molecular identifier (UMI). Heavy chain variable regions were amplified with an IgG-specific nested PCR and amplicons were used for library preparation and illumina MiSeq $2 \times 300$ bp sequencing.

NGS sequence processing. Raw read pre-processing was performed with an inhouse pipeline primarily based on self-written Python scripts, IgBLAST ${ }^{66}$, Clustal Omega $^{67}$ and the pRESTO toolkit ${ }^{68}$. Raw reads were filtered for a mean Phred quality score of 25 and a mean read length of $250 \mathrm{bp}$. UMIs were extracted and IgBLAST was used to pre-annotate reads. Each read pair was annotated with its top $\mathrm{V}$ gene call, its UMI and an additional $18 \mathrm{nt}$ molecular identifier (MID) starting $12 \mathrm{nt}$ after the framework region (FWR) 3. UMIs were grouped and sequences (referred to as 'collisions') were removed if they differed from the most abundant $\mathrm{V}$ gene call or had more than $1 \mathrm{nt}$ difference to the remaining reads in their UMI group. We assumed that reads with the same V gene/MID and an UMI edit distance of $1 \mathrm{nt}$ are more likely to represent reverse transcription (RT), PCR or sequencing errors within the UMI than real different molecules. To rescue those erroneous UMIs, we re-grouped colliding reads and remaining single UMI reads by their MID and re-defined MID groups as the same UMI group, if they shared the same V gene and their UMI had no more than 1 nt difference. UMI groups were aligned with Clustal Omega and then collapsed to build consensus reads. To this end, we weighted each base call by its quality ( 1 - error probability) and generated sums for different base calls over each position to account for both total abundance and the corresponding quality. The base with the highest sum was taken as the consensus base. Paired consensus reads were assembled to one sequence using the pRESTOs AssemblePairs module with a minimal overlap of $6 \mathrm{nt}$. The number of UMI counts per consensus read was plotted against the total number of sequences. This gives a bimodal distribution with a large peak for single reads (UMI found only once) and a broader peak at roughly 20-100 UMIs per consensus read, representing the average oversequencing per molecule. We set an individual cutoff for the minimal UMI number at the intersection of both distributions for each sample $(\mathrm{EV} 01=14, \mathrm{EV} 03=8, \mathrm{EV} 04=6, \mathrm{EV} 05=10)$ to get rid of artificial diversity that mainly comes from PCR and sequencing errors within the UMIs. Assembled full-length sequences were annotated with IgBLAST and only productive sequences with full sequence annotation were kept for downstream analysis. V gene usage, CDR3 length and V gene germline identity distributions were determined from all final sequences without further collapsing.

Cloning, expression and purification of EBOV GP ectodomain for structural studies. The ectodomain of EBOV GP (PDB 5JQB_A) was chemically synthesized (Genscript) to eliminate the mucin-like region and to include a C-terminal trimerization domain of T4 fibritin (foldon) followed by a His-tag as previously reported ${ }^{37}$. The truncated EBOV GP gene was subcloned into a modified pHLsec expression vector (BD Biosynthesis) and transfected using $40 \mathrm{kDa}$ polyethylenimine (PEI-MAX, Polysciences) at $1 \mathrm{mg}$ of plasmid DNA per 11 of HEK293F cells (Invitrogen). To inhibit the formation of complex glycosylation, the mannosidase inhibitor kifunensine (Cayman Chemical) was added to a final concentration of $5 \mu \mathrm{M}$. Media were collected after 6 days and supplemented with $0.02 \%$ (wt/ vol) sodium azide and PMSF. Media were collected and buffer exchanged to TBS (20 mM Tris- $\mathrm{HCl}$ pH 8.0, $150 \mathrm{mM}$ sodium chloride) using a tangential flow filtration system (Merck Millipore). Protein was captured using a HiTrap IMAC FF $\mathrm{Ni}^{2+}$ (GE Healthcare) affinity column followed by size exclusion chromatography (SEC) purification with a Superdex 200 10/300 column (GE Healthcare).

\section{Cloning and purification of anti-EBOV GP Fabs for crystallography. Anti-} EBOV GP IgG clones 3T0331 and 4m0368 were expressed in HEK293F suspension cells (Invitrogen) by co-transfecting the heavy and light chains encoding vectors. Transfections were carried out using PEI-MAX (Polysciences). Media were collected after 6 days of incubation and IgGs were captured using protein-A affinity chromatography (Merck). IgGs were digested using Papain (Merck) with an enzyme and protein ratio of $\sim 1: 20$. Digestion proceeded for $60 \mathrm{~min}$ at $37^{\circ} \mathrm{C}$ in a buffer containing $20 \mathrm{mM}$ cystein- $\mathrm{HCl}$ (Merck) and $10 \mathrm{mM}$ EDTA titered to $\mathrm{pH} 7.0$. Fabs were separated from $\mathrm{Fc}$ fragments by collecting the flowthrough fraction from a protein-A column, followed by SEC on a Superdex 75 10/300 column. Fabs were mixed with EBOV GP ectodomain at a molar ratio of 1:2, and the complex was isolated by SEC on a Superdex 200 10/300.

Crystallization, data collection and structure determination of Fab 3T0331 A mosquito crystallization robot (TTP labs) was used to set 60,120 and $180 \mathrm{nl}$ sitting drops of Fab 3T0331 $\left(11 \mathrm{mg} \mathrm{ml}^{-1}\right)$ with a $120 \mathrm{nl}$ reservoir of commercially available crystallization screens. Crystallization hit for the Fab was identified using a PEGRx HT kit (Hampton). Crystals were obtained in $0.15 \mathrm{M}$ lithium sulfate monohydrate, $0.1 \mathrm{M}$ citric acid pH 3.5, $18 \%$ PEG 6000 at $20^{\circ} \mathrm{C}$. Crystals were briefly soaked in mother liquor solution supplemented with $25 \%$ ethylene glycol for cryo preservation before flash cooling in liquid nitrogen. X-ray diffraction data were collected at the European Synchrotron Radiation Facility (ESRF) at beamline ID23-2 using a Pilatus3 2M detector. Diffraction data were collected to a resolution of $1.56 \AA$ using X-ray radiation at a wavelength of $0.87313 \AA$ at $100 \mathrm{~K}$. Images were indexed, integrated using Xia ${ }^{69}$, and scaled using Aimless ${ }^{70}$. A molecular replacement solution was found using Phaser ${ }^{71}$ with a single Fab in the asymmetric unit, and the model was built and refined in an iterative manner using $\operatorname{Coot}^{72}$ and Phenix refine ${ }^{73} .98 .14 \%$ of the residues in the refined model occupied favored Ramachandran space and an additional $1.86 \%$ of the residues were in allowed Ramachandran space.

Cryo-EM image acquisition, data processing and model building. Complexes of EBOV GP/Fabs $\left(0.4 \mathrm{mg} \mathrm{ml}^{-1}\right)$ were applied to glow-discharged C-flat 2/1$3 \mathrm{Cu}-50$ holey carbon grids (EMS) and plunge frozen in liquid ethane, using a ThermoFisher Vitrobot plunger ( $2.5 \mathrm{~s}$ blotting time, $100 \%$ humidity). Cryo-EM data were collected on a Titan Krios electron microscope (FEI) operated at $300 \mathrm{kV}$. Coma-free alignment was performed with AutoCTF (FEI) and the beam size was $2.2 \mu \mathrm{m}$. Videos were recorded on a Falcon 3EC direct detector (ThermoFisher) and were collected in super-resolution counting mode at a nominal magnification of $\times 96,000$ corresponding to a physical pixel size of $0.85 \AA$. The total dose rate was set to one electron per physical pixel per second and the total exposure time was 29.6 s, resulting in an accumulated dose of 40 electrons per $\AA^{2}$. Each movie was fractionated into 39 frames of $0.76 \mathrm{~s}$. The nominal defocus range was -0.5 to $-2.3 \mu \mathrm{m}$. A total of 437 and 723 videos were recorded for EBOV GP/3T0331 and EBOV GP/4m0368, respectively. 
All data processing was conducted using the cryoSPARC V2 suite ${ }^{74}$. Videos were motion-corrected and contrast transfer functions were fitted using CTFFIND $4^{75}$. Templates for auto picking were derived by two-dimensional (2D) classification of manually picked particles. Following template-based autopicking, a total of 109,407 and 123,880 good particles were selected for reconstruction of EBOV GP/3T0331 and EBOV GP/4m0368, respectively, based on iterative reference-free $2 \mathrm{D}$ classifications. Initial reference maps were calculated using ab initio reconstruction and high-resolution maps were obtained using non-uniform $3 \mathrm{D}$ refinement, while imposing $\mathrm{C} 3$ symmetry. Working maps were locally filtered after calculating local resolution estimates.

Models of EBOV GP/3T0331 and EBOV GP/4m0368 were assembled by rigid-body fitting the crystallographic structure of EBOV GP (PDB 5JQ3) ${ }^{37}$ into electron density maps using UCSF chimera ${ }^{76}$ as well as of the $V_{H} V_{L}$ portions from the crystallographic structure of 3T0331 and from a computationally modeled $4 \mathrm{~m} 0368$. The latter structure was modeled using the ABpredict tool ${ }^{38}$. Both structures were then refined using the Phenix real-space refinement tool ${ }^{73}$ and manually fitted into electron density maps using $\operatorname{Coot}^{72}$ in an iterative process.

Estimation of antibody pharmacokinetic profiles in vivo. NOD.Cg-Rag1 $1^{\text {tmlmom }}$

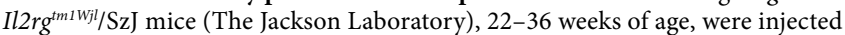
intravenously with $0.5 \mathrm{mg}$ of antibody $\left(200 \mu \mathrm{l}\right.$ at a concentration of $2.5 \mathrm{mg} \mathrm{ml}^{-1}$ in PBS, tail vein) on day 0 . Total human IgG serum concentrations were determined over a period of 14 days by ELISA as described previously ${ }^{77}$. Briefly, high binding ELISA plates (Corning) were coated overnight with goat anti-human IgG (Jackson ImmunoResearch) and blocked with 2\% BSA, $1 \mu \mathrm{M}$ EDTA and $0.1 \%$ Tween-20 in PBS for $2 \mathrm{~h}$. Serum and standard samples were incubated for $90 \mathrm{~min}$, followed by HRP-conjugated goat anti-human IgG (Jackson ImmunoResearch) for $90 \mathrm{~min}$. Absorbance at $415 \mathrm{~nm}$ was determined (Tecan) after the addition of ABTS (Thermo Fisher). Plates were washed with $0.05 \%$ Tween-20 in PBS between each step. The absence of detectable human IgG was confirmed in all mice using pre-infusion serum samples. Mouse experiments were authorized by the State Agency for Nature, Environment and Consumer Protection (LANUV) of North Rhine-Westphalia.

Quantification and statistics. Flow cytometry analysis and quantifications were performed with FlowJo10. EC $_{50}$ values, means and standard deviations of serum and antibody reactivities were calculated from technical replicates $(n=2$ per sample; serum samples $n=6$ for EV, $n=7$ for EVD survivor) using GraphPad Prism (version 7). Tests for significance were conducted using GraphPad Prism and R (R Core Team, 2018). Differences in survivor and rVSV-ZEBOV vaccinees (serum response and neutralization) were tested by two-tailed unpaired $t$-tests. The frequency increase of the 58 different $V$ genes between EBOV GP $\Delta$ TMspecific B cell sequences and the IgG repertoire (Fig. $2 \mathrm{~g}$ ) was analyzed by normalizing each data set (EBOV GP $\Delta$ TM-specific and IgG repertoire) on relative frequencies for each of the four vaccinees and computing the differences among relative frequencies. To test the significance for the single $\mathrm{V}$ genes, two data sets were generated. Set $\mathrm{A}$ included all four differences for the $\mathrm{V}$ gene of interest, and set B included 57 differences, one for each other $\mathrm{V}$ gene, where the subject was chosen uniformly at random. A one-sided Mann-Whitney U (rank sum) test was applied on the two sets to determine whether set A was stochastically greater than set $\mathrm{B}$. To account for the random sampling in the construction of set $\mathrm{B}$, this setup was repeated $10^{3}$ times and the averaged resulting $P$ values were used with $\alpha=0.05$ and Bonferroni correction for multiple testing. The frequency increase of $\mathrm{V}$ genes of EBOV-neutralizing antibodies (Fig. 6a) was tested for significance using a binomial test under the null hypothesis of equal $\mathrm{V}$ gene frequency, $\alpha=0.05$ and Bonferroni correction. The sequence similarity of CDRH3 was determined by Geneious software using BLOSUM (BLOcks Substitution Matrix) with residue-specific and hydrophilic gap penalties (open gap penalty of 10 , extended gap penalty 0.2 ). The calculations of phylogenetic trees and sequence alignments were done with Geneious (version 10). To compare the heterogeneity of sequences within the same donor with that among different donors, pairwise alignment scores among all sequences were computed by needle (with default parameters) and grouped accordingly. The difference of both distributions of alignment scores was assessed with a Mann-Whitney U test. Sequence logo plots were generated with WebLogo $3^{78}$ followed by minor adjustments.

Reporting Summary. Further information on research design is available in the Nature Research Reporting Summary linked to this article.

\section{Data availability}

In this manuscript, we present the data necessary to interpret and replicate our results. All experiments were performed as described in the Methods. Newly developed primer sets (Kreer et al., submitted) are available from the corresponding author upon request. All antibody sequences are currently being processed to be uploaded to GenBank. EM structures of EBOV GP with the Fab fragments of monoclonal antibodies 3T0331 (PDB 6QD7) and 4m0368 (PDB 6QD8) have been deposited at PDB.

\section{Code availability}

All programs used for data analysis are described in the Methods. Sequence processing is described in detail and more information on the specific programming can be obtained on request.

\section{References}

61. Mittler, E., Schudt, G., Halwe, S., Rohde, C. \& Becker, S. A fluorescently labeled Marburg virus glycoprotein as a new tool to study viral transport and assembly. J. Infect. Dis. 218, S318-S326 (2018).

62. Maruyama, T. et al. Ebola virus can be effectively neutralized by antibody produced in natural human infection. J. Virol. 73, 6024-6030 (1999).

63. Gaebler, C. et al. Isolation of HIV-1-reactive antibodies using cell surfaceexpressed gp160 $\Delta$ c(BaL.). J. Immunol. Methods 397, 47-54 (2013).

64. Tiller, T. et al. Efficient generation of monoclonal antibodies from single human B cells by single cell RT-PCR and expression vector cloning. J. Immunol. Methods 329, 112-124 (2008).

65. von Boehmer, L. et al. Sequencing and cloning of antigen-specific antibodies from mouse memory B cells. Nat. Protoc. 11, 1908-1923 (2016).

66. Ye, J., Ma, N., Madden, T. L. \& Ostell, J. M. IgBLAST: an immunoglobulin variable domain sequence analysis tool. Nucleic Acids Res. 41, W34-W40 (2013).

67. Sievers, F. et al. Fast, scalable generation of high-quality protein multiple sequence alignments using Clustal Omega. Mol. Syst. Biol. 7, 539 (2011).

68. Vander Heiden, J. A. et al. pRESTO: a toolkit for processing high-throughput sequencing raw reads of lymphocyte receptor repertoires. Bioinformatics 30, 1930-1932 (2014).

69. Winter, G. xia2: an expert system for macromolecular crystallography data reduction. J. Appl. Crystallogr. 43, 186-190 (2010).

70. Evans, P. R. \& Murshudov, G. N. How good are my data and what is the resolution? Acta Crystallogr. D 69, 1204-1214 (2013).

71. McCoy, A. J. et al. Phaser crystallographic software. J. Appl. Crystallogr. 40, 658-674 (2007).

72. Emsley, P., Lohkamp, B., Scott, W. G. \& Cowtan, K. Features and development of Coot. Acta Crystallogr. D 66, 486-501 (2010).

73. Adams, P. D. et al. PHENIX: a comprehensive python-based system for macromolecular structure solution. Acta Crystallogr. D 66, 213-221 (2010)

74. Punjani, A., Rubinstein, J. L., Fleet, D. J. \& Brubaker, M. A. cryoSPARC: algorithms for rapid unsupervised cryo-EM structure determination. Nat. Methods 14, 290-296 (2017).

75. Rohou, A. \& Grigorieff, N. CTFFIND4: fast and accurate defocus estimation from electron micrographs. J. Struct. Biol. 192, 216-221 (2015).

76. Pettersen, E. F. et al. UCSF Chimera-a visualization system for exploratory research and analysis. J. Comput. Chem. 25, 1605-1612 (2004).

77. Klein, F. et al. HIV therapy by a combination of broadly neutralizing antibodies in humanized mice. Nature 492, 118-122 (2012).

78. Crooks, G. E., Hon, G., Chandonia, J. M. \& Brenner, S. E. WebLogo: a sequence logo generator. Genome Res. 14, 1188-1190 (2004). 


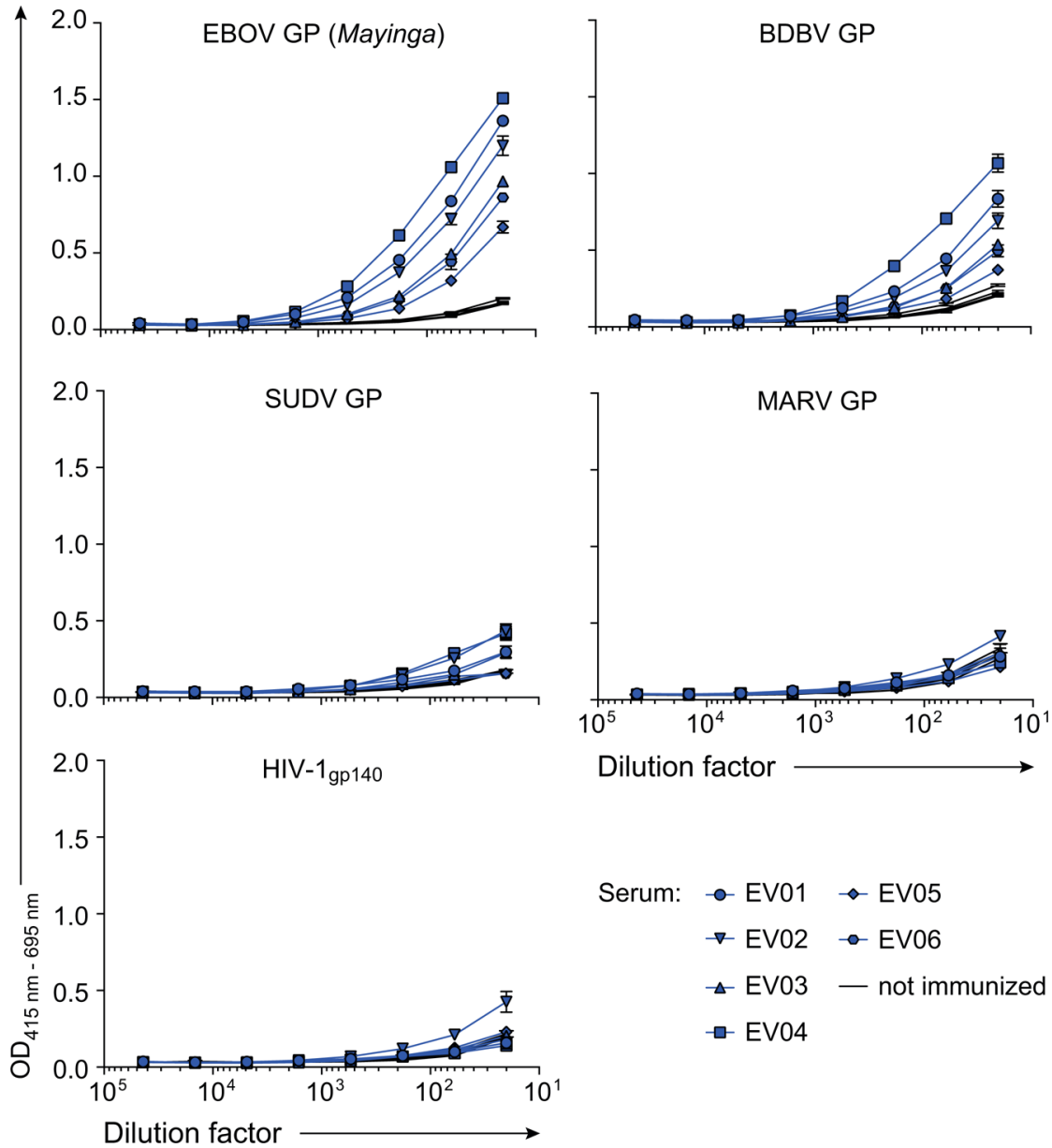

Extended Data Fig. 1 | Serum response to EBOV, BDBV, SUDV, and MARV in rVSV-ZEBOV-vaccinated individuals (related to Fig. 1). Serum samples of rVSV-ZEBOV-vaccinated individuals (EV01-EV06) were tested for cross-reactivity against the Ebolavirus species EBOV, BDBV, SUDV, and Marburg virus (MARV). HIV-1 gp140 was used as a control. Means and standard errors of the means (SEM) of technical duplicates are shown. 
a
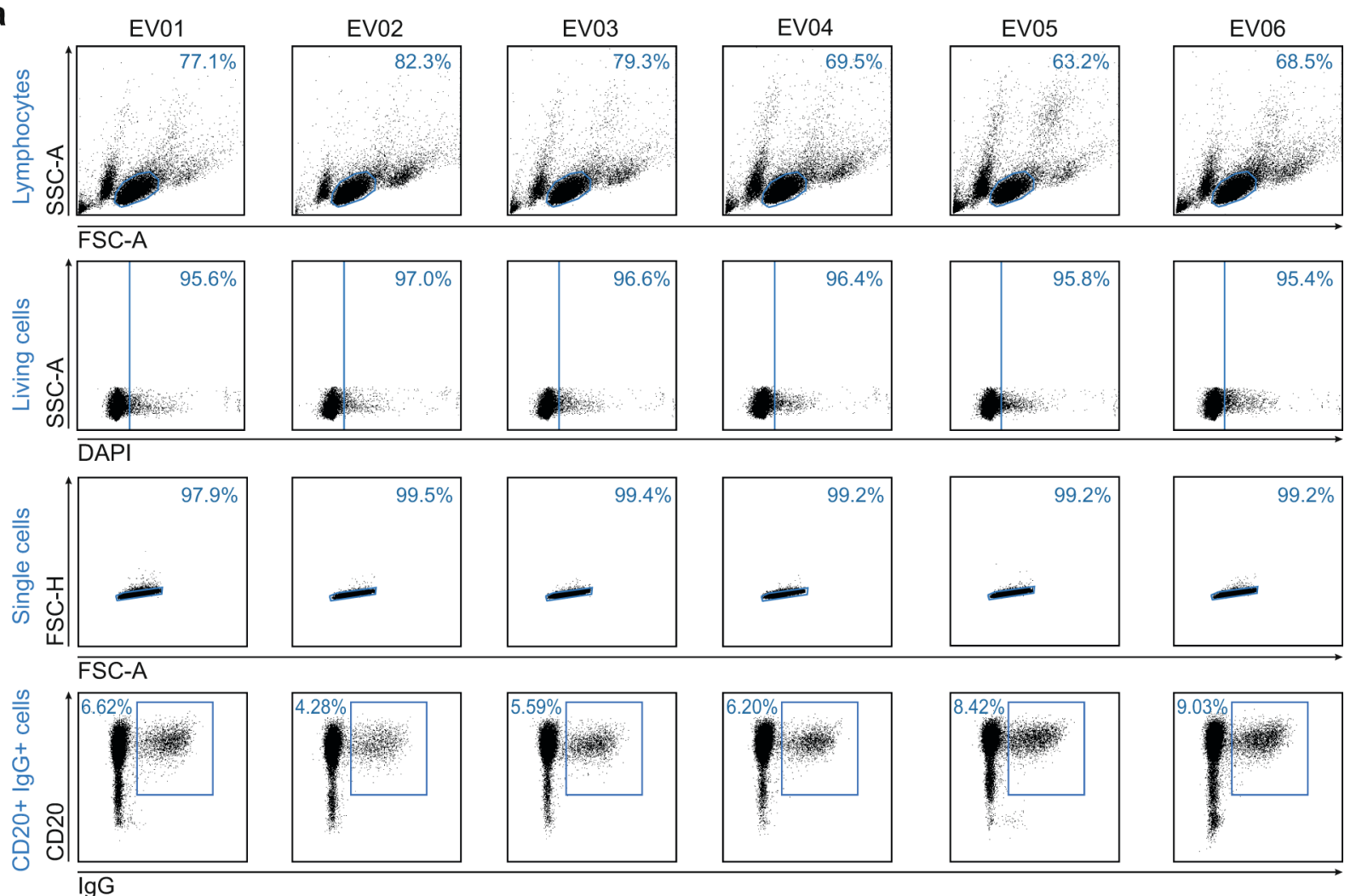

b

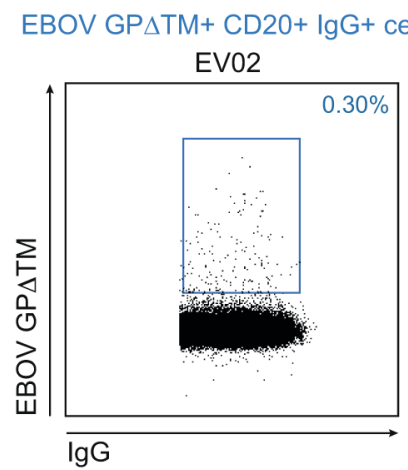

C
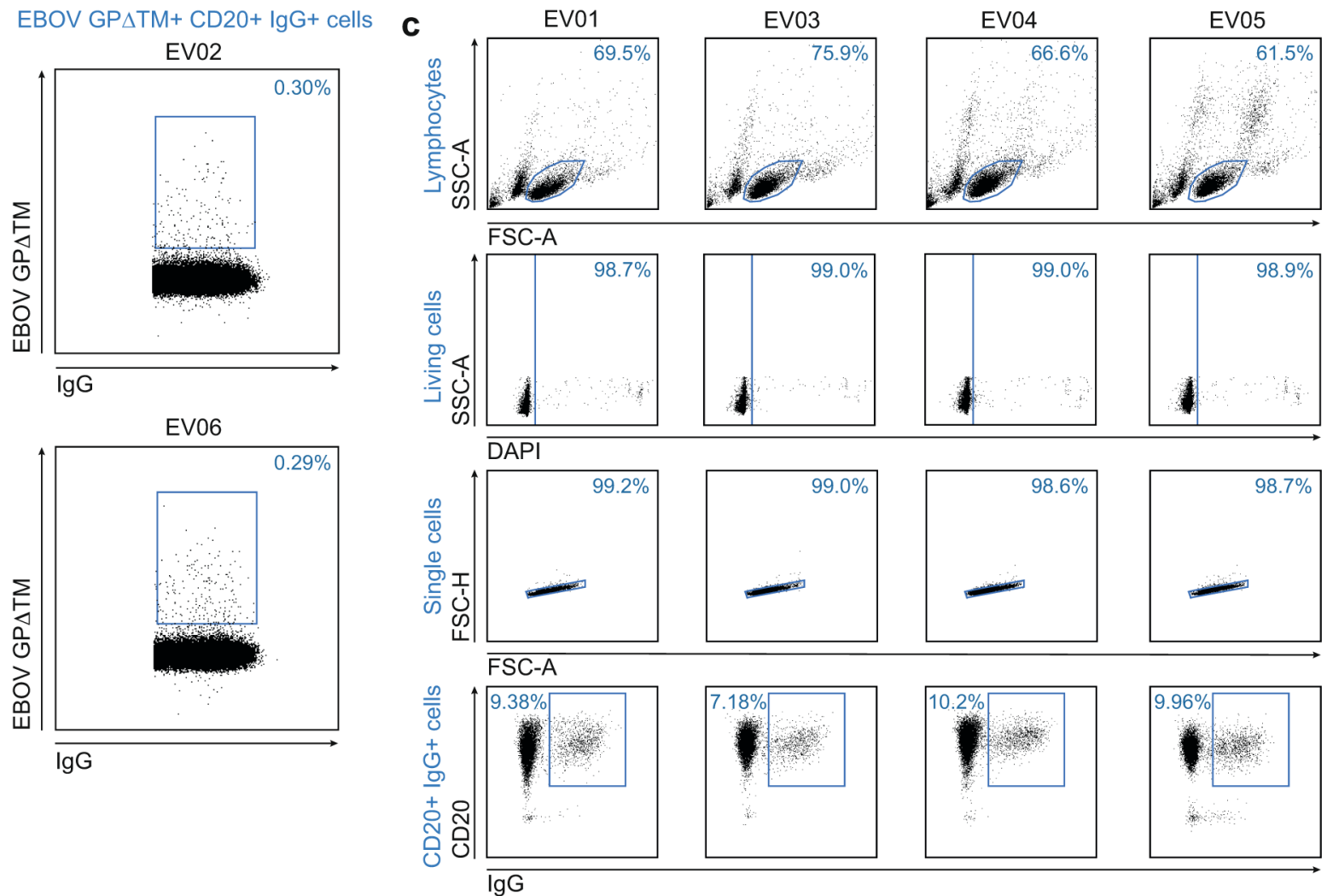

Extended Data Fig. 2 | Gating strategy for B cells from rVSV-ZEBOV-vaccinated individuals (related to Fig. 2). (a) Gating strategy for flow cytometric analysis. CD19-enriched (MACS) PBMCs of rVSV-ZEBOV-vaccinated donors (EV01-EV06) were gated for lymphocytes $->$ live cells $->$ single cells. $\mathrm{CD}_{2} \mathrm{O}^{+} / \mathrm{lgG}+\mathrm{B}$ cells were selected for sort of EBOV-specific B cells (see Fig. 2). (b) Frequencies of EBOV G $\triangle T M$-specific CD20 $/ / \mathrm{lgG}+$ cells of EV02 and EVO6 selected as in (a). Corresponding plots of EV01, EV03, EVO4 and EV05 are shown in Fig. 2a. (c) Gating strategy to select CD20+1/gG+ cells for B cell repertoire analyses by NGS. In total 250,000 CD20+/lgG+ B cells were sorted for each rVSV-ZEBOV-vaccinated donor. 
a

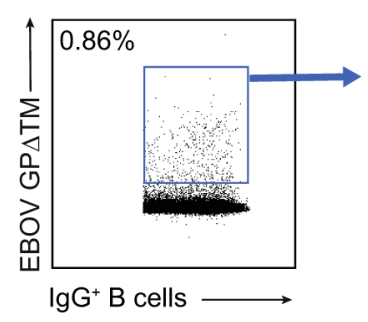

b

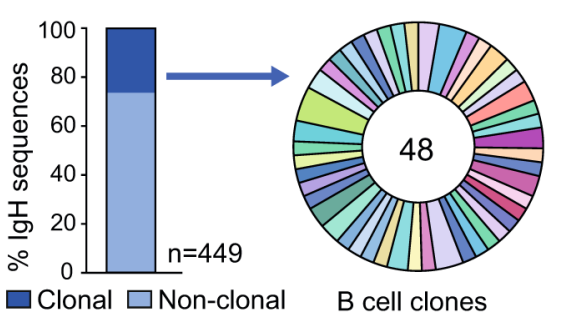

d

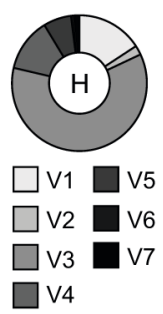

e

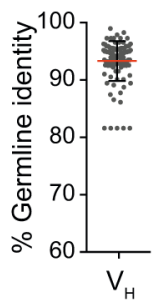

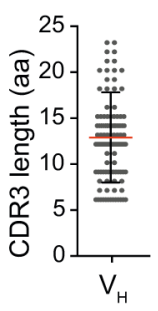

$\mathbf{f}$

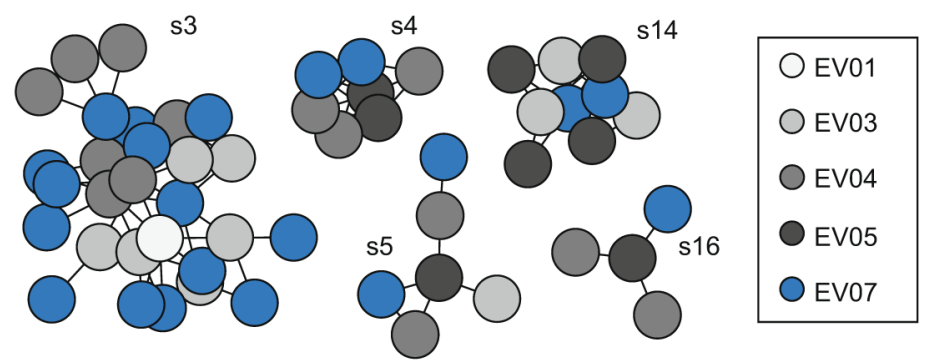

$\mathbf{g}$

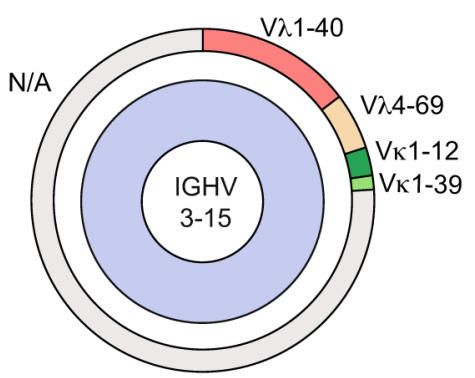

h
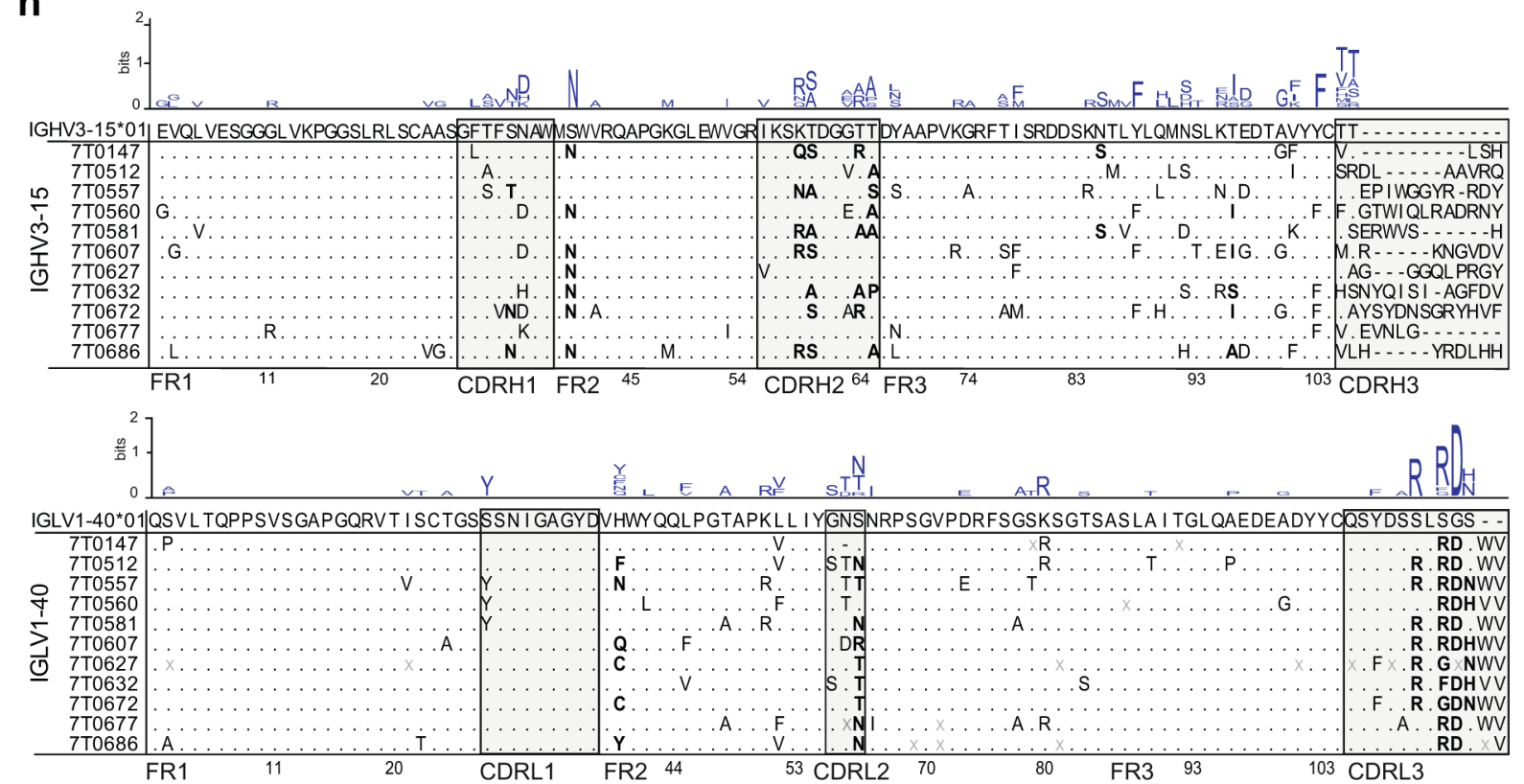

CDRH1 FR2

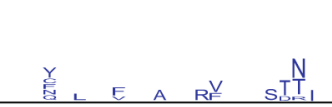

N
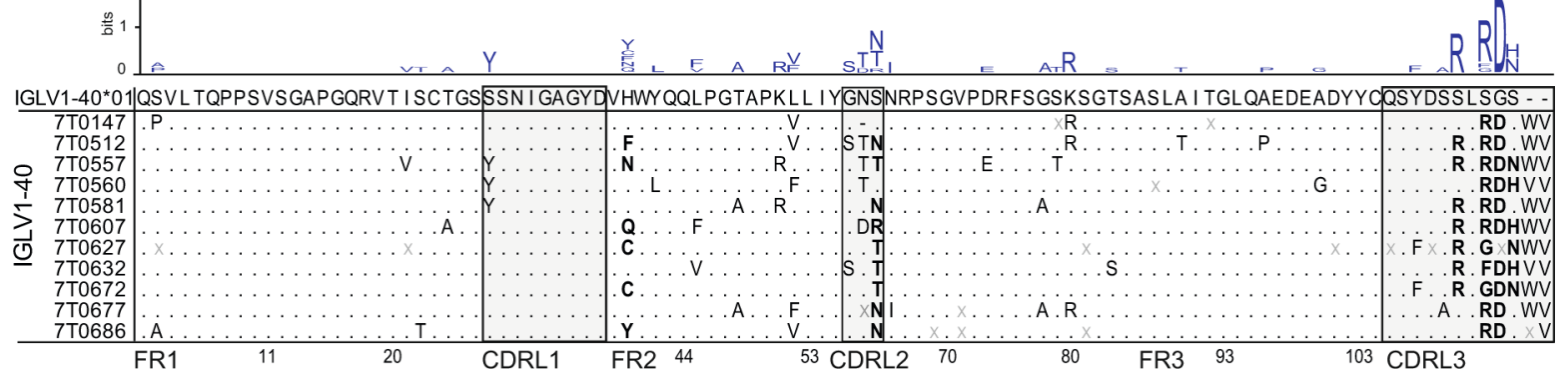

Extended Data Fig. 3 | B cell response of an individual EV07 vaccinated with $2 \times 10^{7}$ p.f.u. rVSV-ZEBOV (related to Figs. 2, 3 and 5). (a) Flow cytometry on $\mathrm{CD}_{19}{ }^{+}$magnetic bead-enriched PBMCs from rVSV-ZEBOV-vaccinated individual (EV07) $(n=1)$. Gate captures the EBOV GP $\triangle T M-r e a c t i v e ~ p o p u l a t i o n$ from lgG ${ }^{+} / \mathrm{CD}_{2} \mathrm{O}^{+} \mathrm{B}$ cells that were selected for single cell sorting. See also Fig. 2b Abundance of amplified clonal (dark blue) and non-clonal (light blue) immunoglobulin (Ig) sequences. (c) Identified B cell clonotypes (total number in the center of pie). Each slice represents a unique B cell clone (size proportional to the number of clonal members). (d) Distribution of $V$ gene families in heavy chains of all unique clones. (e) Germline identity (left) and CDR3 aa lengths (right) of VH of in (a) identified clonal sequences $(n=449)$. Means are shown in red and SD in black. ( $f$ ) Contribution of EV07 to shared sequences between vaccinees. Connecting lines between sequences (circles) from different donors indicated shared VH gene and at least $75 \%$ CDR3 aa identity. See also Fig. 3. (g) Light chain combination of IGHV3-15 antibody sequences. $\lambda$-chains are displayed in shades of orange, $\kappa-c h a i n s$ in shades of green, and not analyzed chains (N/A) in gray. See also Fig. 3 and Extended Data Fig. 4. (h) Alignment of aa sequences of obtained IGHV3-15 (top)/IGLV140 (bottom) antibodies in comparison to germline. For positions labeled with more than $50 \%$ of the sequences mutated in Fig. 5, aa are displayed in bold letters. Sequence Logo plots were generated by WebLogo 3 with minor alterations. 
a

$\mathrm{CD} 20+\lg \mathrm{G}+$ cells

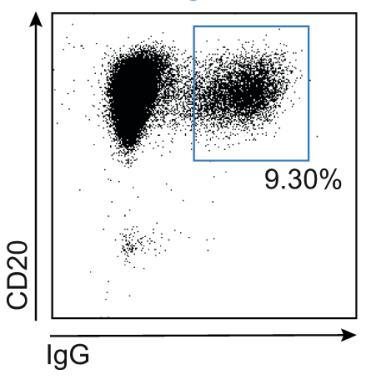

EBOV GP+ cells

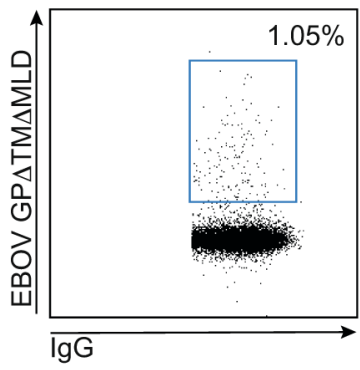

b

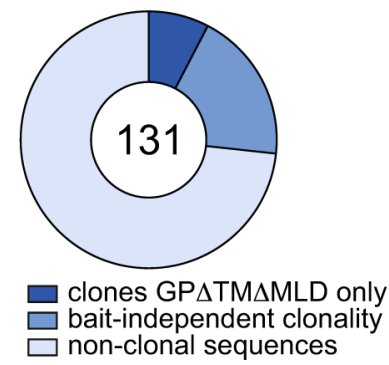

C

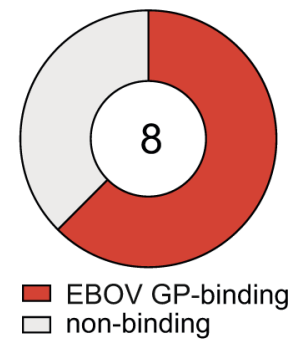

Extended Data Fig. 4 | Single B cell analysis and characterization of EBOV GP-specific B cells using an alternative bait EBOV GP $\triangle T M \triangle M L D$ (related to Figs. 2 and 4 and Supplementary Table 6). (a) EBOV GP-specific B cells from EV04 were isolated using DyLight488-conjugated EBOV GP $\Delta T M \triangle M L D$ (strain Makona). CD19+ magnetic bead-enriched (MACS) PBMCs were analyzed by FACS $(n=2)$ and lgG ${ }^{+} / \mathrm{CD}^{+} \mathrm{O}^{+}$B cells were selected (left) to sort EBOV GP $\triangle T M \triangle M L D$-reactive cells (right). (b) Fractions of the 137 sequences obtained are colored in light blue for non-clonal, medium blue for clonal and bait-independent, and dark blue for sequences obtained with EBOV GP $\triangle T M \triangle M L D$ only. (c) Reactivity of all antibodies identified by EBOV GP $\Delta T M \triangle M L D$ in binding against EBOV GP $\triangle T M$. 
a

\begin{tabular}{|c|c|c|c|c|}
\hline & IGHV & $\mathrm{V}_{\mathrm{H}} \mathrm{CDR} 3$ & IGLV & $\mathrm{V}_{\mathrm{L}} \mathrm{CDR} 3$ \\
\hline \#1 & $3-13$ & $\begin{array}{l}\text { ARAVFGSSAFDI } \\
\text { VRAIFGSHAFDI } \\
\text { VRAIFGSHAFDI }\end{array}$ & $\begin{array}{l}3-20 \kappa \\
3-20 \kappa \\
3-20 \kappa\end{array}$ & $\begin{array}{l}\text { QQYGSSPLT } \\
\text { OOYGTSPLT } \\
\text { OOYGSAPLT }\end{array}$ \\
\hline \#2 & $3-23$ & 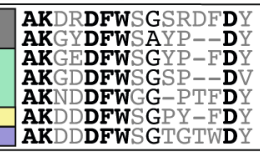 & $\begin{array}{l}1-51 \lambda \\
1-47 \lambda \\
1-47 \lambda \\
1-51 \lambda \\
1-51 \lambda \\
1-51 \lambda \\
\text { n.d. }\end{array}$ & 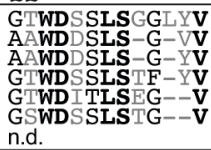 \\
\hline \#3 & $3-23$ & $\begin{array}{l}\text { AKEKDFWNGYYIDY } \\
\text { AKDSTFWNGNYFD } \\
\text { AKEYDFWNGRYFD } \\
\text { AKEYDFWNGRYFDC }\end{array}$ & $\begin{array}{c}1-51 \lambda \\
1-51 \lambda \\
\text { n.d. } \\
1-51 \lambda\end{array}$ & $\begin{array}{l}\text { GTWDISLNAWV } \\
\text { GTWDGSLSVGV } \\
\text { n.d. } \\
\text { GTWDISLNVGV }\end{array}$ \\
\hline \#4 & $3-48$ & $\begin{array}{l}\text { ARAAADYNYGMDV } \\
\text { ARAPRDLYYAMDF } \\
\text { ARATVDYYAMDV } \\
\text { ARAPRDYYYGMDV } \\
\text { ATMNRDYYYAMDI }\end{array}$ & $\begin{array}{c}1-40 \lambda \\
1-40 \lambda \\
\text { n.d. } \\
\text { n.d. } \\
1-40 \lambda\end{array}$ & $\begin{array}{l}\text { QSYDDTLSGWV } \\
\text { QTYDDSLSGWV } \\
\text { n.d. } \\
\text { n.d. } \\
\text { QSYDDSLSGYV }\end{array}$ \\
\hline \#5 & $4-34$ & $\begin{array}{l}\text { ARHAHSGMDV } \\
\text { ARHGHTGMDV } \\
\text { ARHGHTGMDV } \\
\text { ARHGHTGMDV }\end{array}$ & $\begin{array}{l}3-20 \kappa \\
2-28 \kappa \\
2-28 \kappa \\
3-10 \lambda\end{array}$ & $\begin{array}{l}\text { OOYGSSPLT } \\
\text { MOALQT PLT } \\
\text { MHALQT PLT } \\
\text { YSTDNSGSHRV }\end{array}$ \\
\hline
\end{tabular}

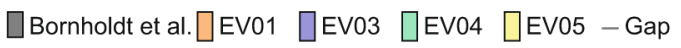
aa similarity: oidentical

- similar (similar chemical characteristics)

- different b

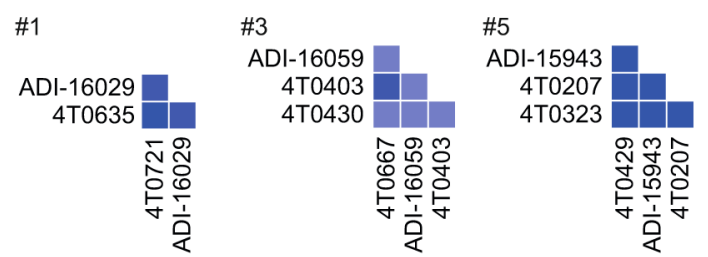

\#2

\#4
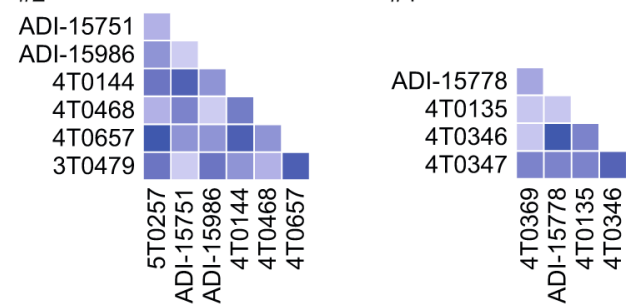

Similarity index (\%):

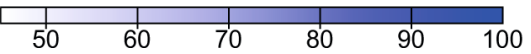

C

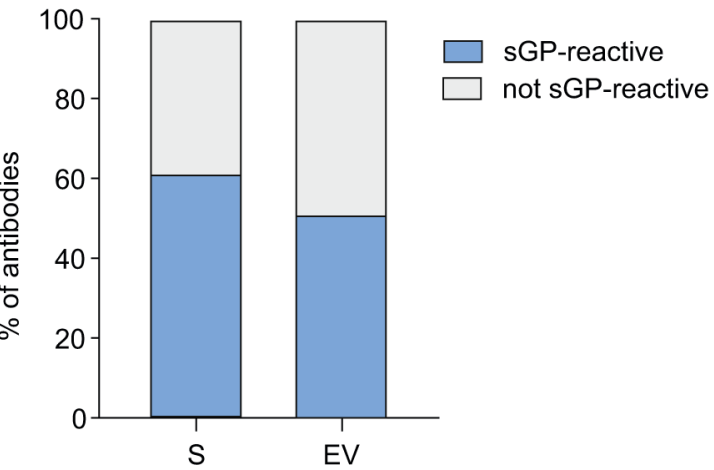

d

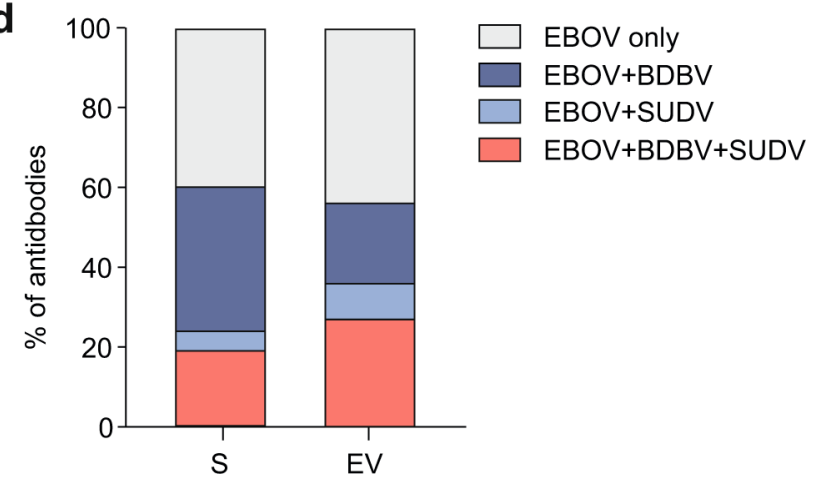

Extended Data Fig. 5 | Comparison of rVSV-ZEBOV-induced and published EVD survivor antibodies (related to Fig. 3). (a) Comparison of IgH aa sequences from rVSV-ZEBOV-vaccinated individuals (EV) and EVD survivors (Bornholdt et al. ${ }^{14}$ ) revealed groups of sequences sharing $V$ genes and CDR3 aa characteristics. Analysis was performed as described in Fig. 3. Colored bars represent different EV individuals (orange, violet, green and yellow) and EVD survivor-derived sequences (gray). Black, dark gray, and light gray letters of CDR3 aa indicate identical, chemically comparable, and different aa, respectively. Chemical characteristics were defined by grouping aa into non-polar, polar, acidic, basic and aromatic aa. (b) Color matrices showing sequence similarities were obtained through pairwise comparison of all CDRH3 sequences within respective groups on aa level using BLOSUM (BLOcks Substitution Matrix) with Geneious software. Comparison of EBOV sGP-reactivity (c) and cross-reactivity (d) of EV- and EVD survivor-isolated mAbs as determined by ELISA or as reported previously (Bornholdt et al. ${ }^{14}$ ). Color scheme as in Fig. 3. 
a

\begin{tabular}{|c|c|c|c|c|c|c|c|}
\hline & & & & 50 & & & \\
\hline GP1 & Head/base structure & Gly-Cap & MLD & GP2 & IFL & HR1+2 & MPER \\
\hline
\end{tabular}

EBOV GP $\triangle T M \triangle M L D$

\begin{tabular}{|c|c|c|c|c|c|c|}
\hline GP1 & Head/base structure & Gly-Cap & GP2 & IFL & HR1+2 & MPER \\
\hline
\end{tabular}

\section{EBOV sGP}

b

EV01

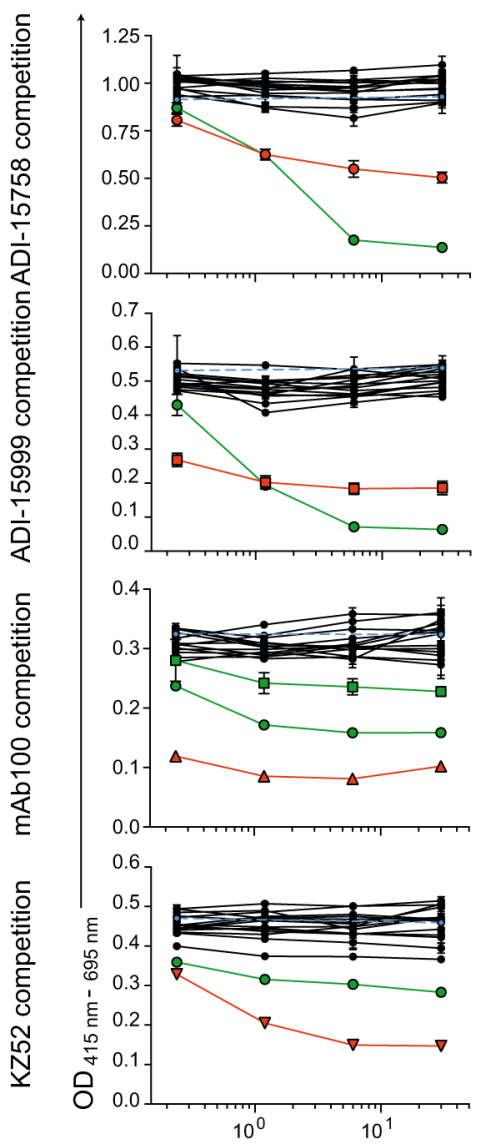

EV03
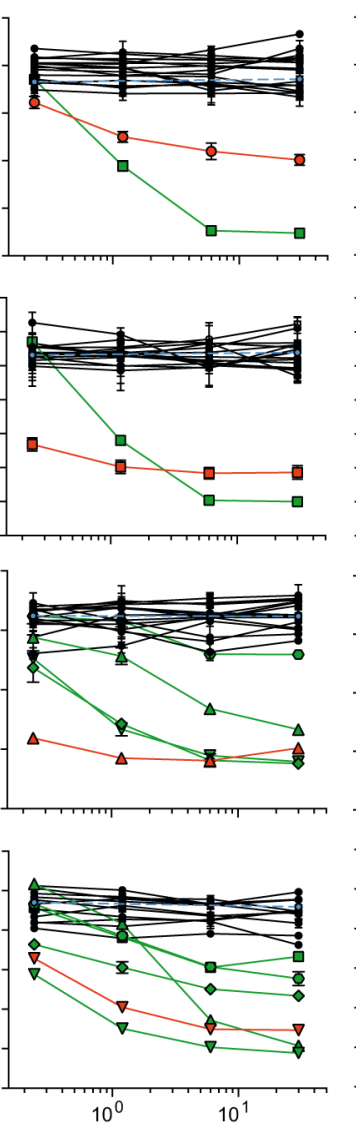

EV04
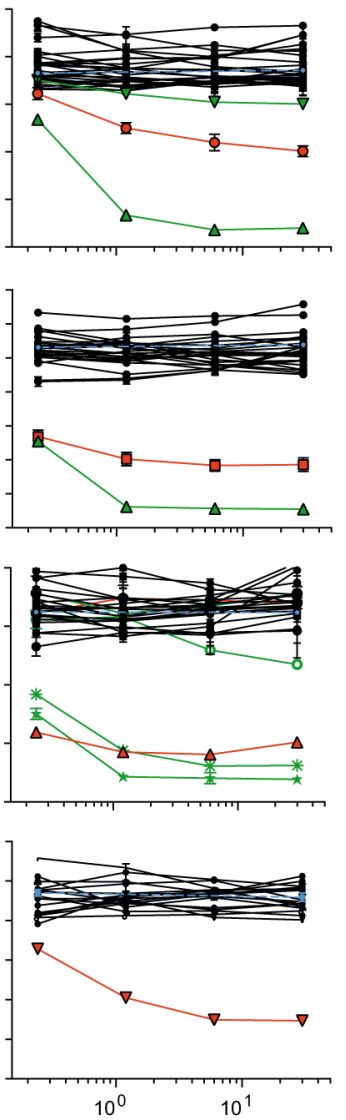

EV05
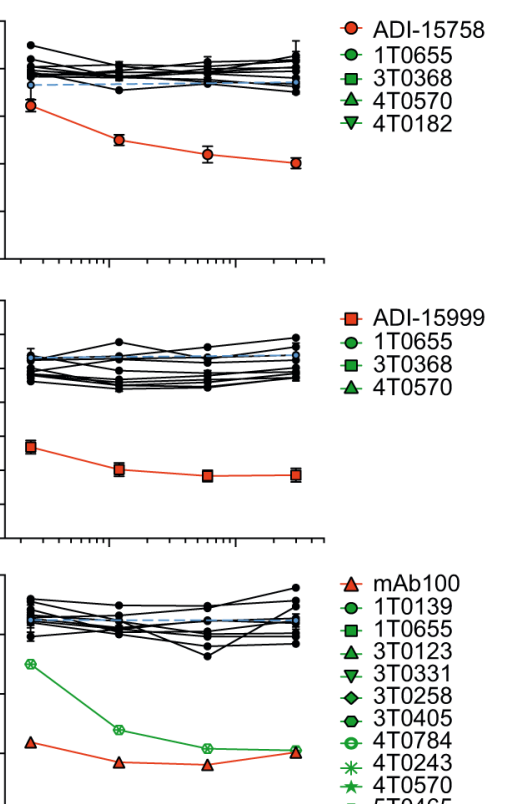

$\triangle \mathrm{mAb} 100$ - 1 T0139 - 1 T0655 $\triangle$
$-3 T 0123$
$-3 T 0331$ $\checkmark$ 3T0258 - 3 TT0405 - 4T0784 * 4T0243 * 4T0570 5T0465

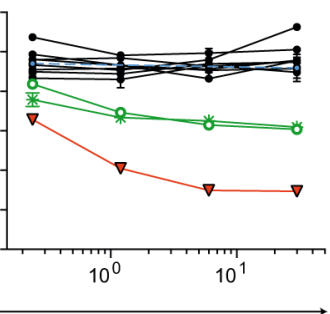

$\nabla \mathrm{KZ} 52$ - 1 T0139 - 3 T0553 $\triangle 3 T 0123$ $\nabla$ 3T0331 $\checkmark$ 3T0258 - 3T0405 - $5 T 0465$
* 5T0377

Antibody concentration $(\mu \mathrm{g} / \mathrm{ml})$

-.. positive binding

- reference antibody

— competing antibody

- no competiton

Extended Data Fig. 6 | Epitope mapping of rVSV-ZEBOV-induced antibodies (related to Fig. 4). (a) Schematic representation of constructs produced to determine target sites of EBOV-specific mAbs. EBOV GP $\triangle T M$ (top; lacking transmembrane domain residues 651-676), EBOV GP $\Delta$ TM $\triangle M L D$ (bottom left; lacking additionally the mucin-like domain [MLD] residues 313-463) and EBOV sGP (bottom right; secreted GP) with the C-terminal end being structurally different from GP1 (light gray). GP1 and GP2 are shown in blue and yellow, respectively. GP1 contains the head/base structure, glycan cap (Gly-cap),

MLD and the C-terminal end of GP1 (residues 464-501). GP2 contains the internal fusion loop (IFL), heptad repeats 1 and 2 (HR1+2), and the membraneproximal external region (MPER). Lines represent disulfide bridges connecting GP subunits. (b) Results of antibody competition against reference mAbs KZ52, mAb100, ADI-15758, and ADI-15999 for binding to EBOV GP $\Delta$ TM. Curves represent all isolated EBOV GP $\Delta$ TM-specific donors. Red curves indicate self-competition and dashed blue curves represent binding of reference $m A b s$ without competition. mAbs with competition are highlighted in green. Competing mAbs were used with a concentration of $0.5 \mu \mathrm{g} / \mathrm{ml}$. Inhibition $>25 \%$ was determined as competition. Error bars show SEM of means of technical duplicates. 
a

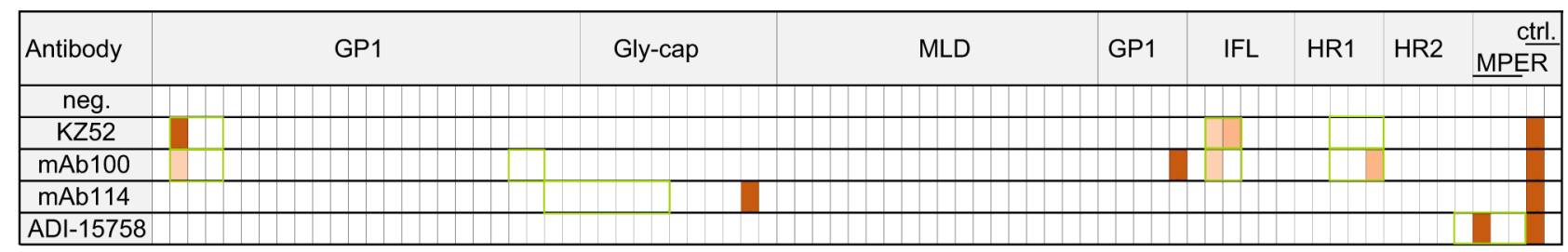
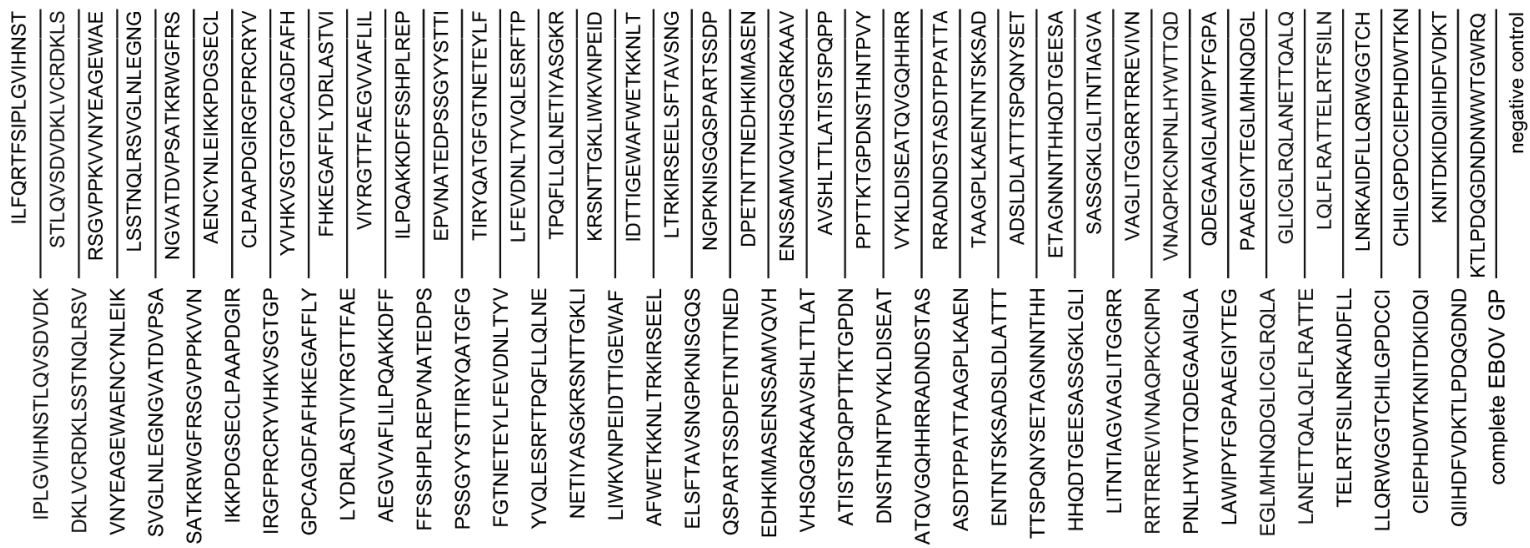

peptides containing described epitope sequences

b

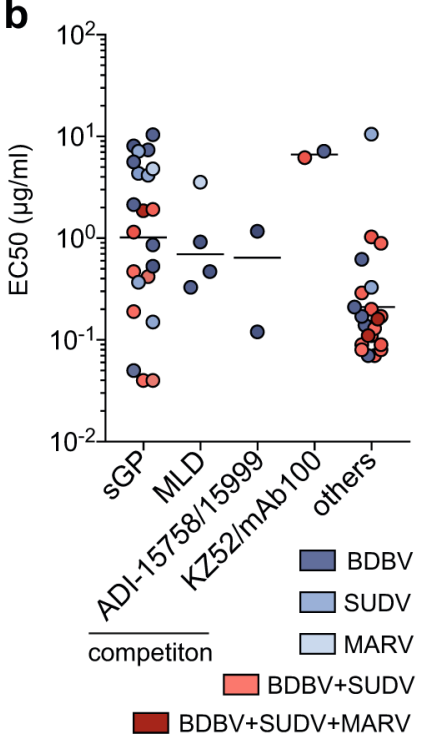

C

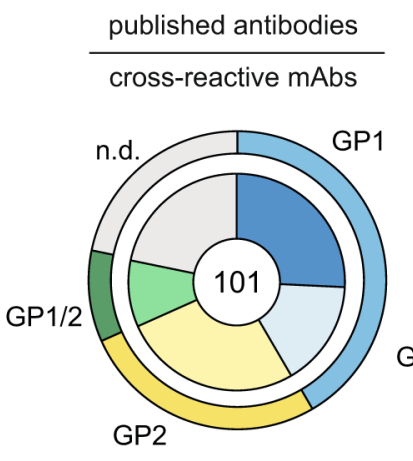

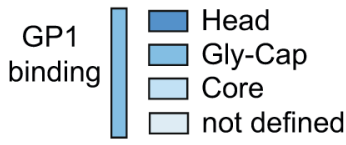

rVSV-ZEBOV-induced antibodies
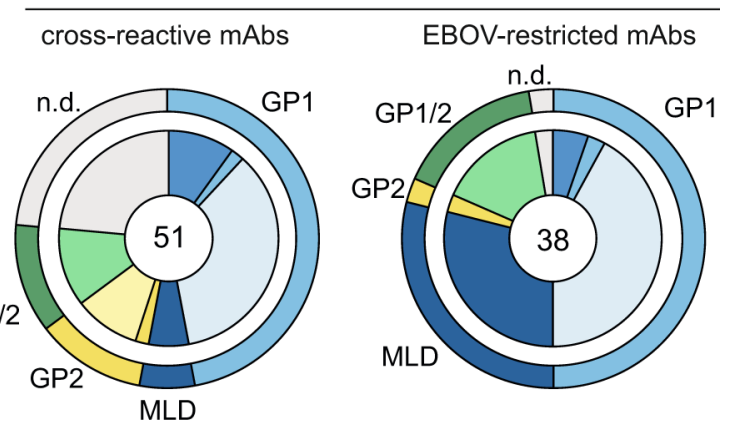

GP2 $\square \mathrm{IFL}$ binding $\square$ HR1+2/MPER

MLD

GP1/2 $\square$ Base/KZ52 competition binding $\square$ GP1/2

Extended Data Fig. 7 | Epitope mapping of cross-reactive antibodies (related to Fig. 4). (a) Peptide library screening results of used reference antibodies. EBOV GP $\triangle T$ TM (Makona) protein was split in 80 peptides of 18 aa length and an overlap of 10 aa. Signals of an OD >0.125 were defined as positive binding (orange-red). Published target epitope aa sequences of the reference mAbs KZ52, mAb100, mAb114, and ADI-15758 were indicated in green contours (Davidson et al., 2015; Lee et al. ${ }^{35}$; Misasi et al. ${ }^{42}$; Ripoll et al., 2017). See also Supplementary Table 5. Representative data of two independent experiments are shown. (b) Epitope distribution of cross-reactive antibodies determined by truncated GP-binding and competition ELISA. Cross-reactivity is indicated by colors. For cross-reactive antibodies best EC50 values are displayed. Means are indicated by lines [ $n=22$ (sGP), 4 (MLD), 2 (ADI-15758-15999), 2 (KZ52/mAb100) and 21 (others)] (c) Left: Epitopes of published, cross-reactive antibodies (Bornholdt et al. ${ }^{14}$, Flyak et al. ${ }^{15}$, and Gilchuk et al. 2018). Right: Summary of epitope distribution of cross-reactive and EBOV-restricted antibodies induced by rVSV-ZEBOV vaccination. Epitopes were determined by ELISA (Fig. 4c,d, Extended Data Fig. 6) and by peptide library screening (a). Total numbers of analyzed mAbs is displayed in the center of the pie. See also Supplementary Table 4. 
a
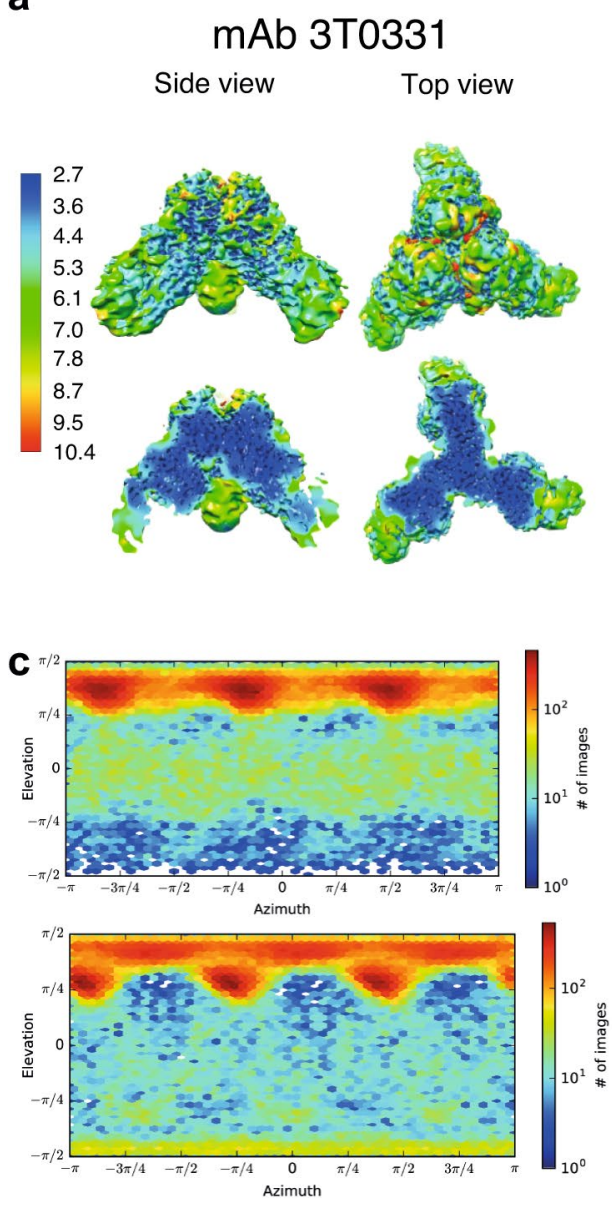

d

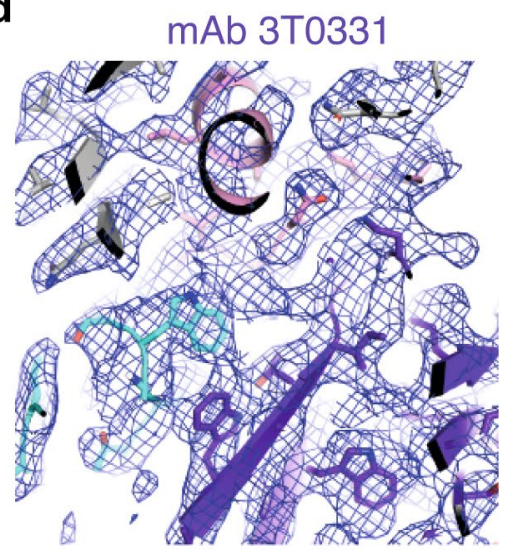

b

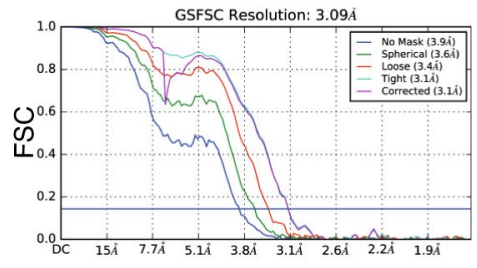

Resolution

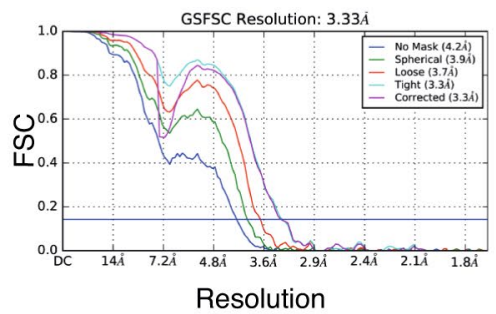

Resolution

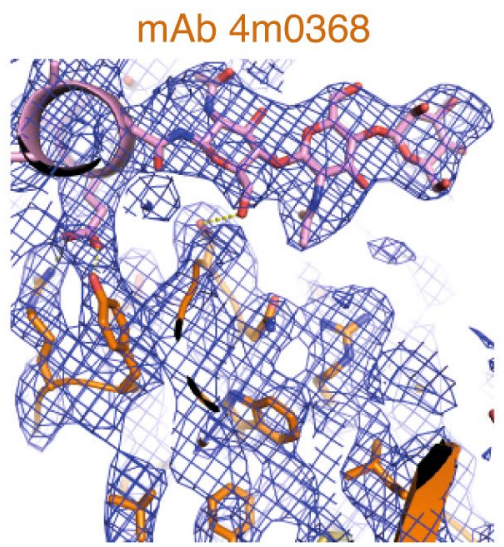

e

EBOV GP/3T0331

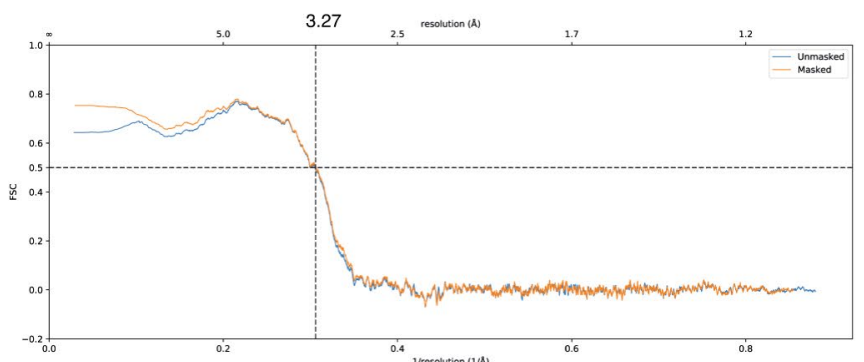

\section{EBOV GP/4m0368}

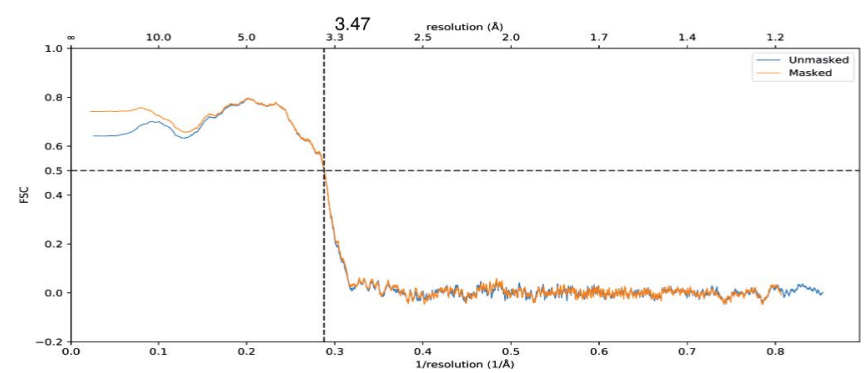

Extended Data Fig. 8 | Cryo EM of rVSV-ZEBOV-induced antibodies (related to Fig. 6). (a-c) Map reconstruction of the EBOV GP/3TO331 complex and EBOV GP/4m0368 complex. (a) Local resolution estimate of the density maps of 3 T0331 (left) and 4m0368 (right). Side and top views are shown on left and right sides, respectively. Slices of the maps at the side and top views orientations are shown in the lower panel. The coloring is based on the local resolution according to the scale shown on the left side. (b) 3T0331 (top) and 4m0368 (bottom) Fourier shell correlation between two half maps as a function of resolution. The resolution value for FSC $=0.143$ is indicated. (c) Angular coverage for the reconstruction of the EBOV GP/3T0331 structure (top) and EBOV GP/4m0368 structure (bottom). Number of images at each angle is indicated according to the scale-bar on the right. (d-e) Electron density - Quality of electron density at the interface between the mAbs and their epitopes. (d) A slice of the EBOV GP/3T0331 model. Electron density map is shown as blue mesh at $\mathrm{s}=7$. Electron density for side-chains is clearly visible (left). A slice of the EBOV GP/4m0368 model. Electron density map is shown as blue mesh at $\mathrm{s}=7$. Electron density for the $\mathrm{N}$-linked glycan as well as various side chains is evident (right). (e) Model_FSC - Model map correlation. Model-map Fourier shell correlation for EBOV GP/3T0331 (left) and EBOV GP/4m0368 (right). 
a

GP1

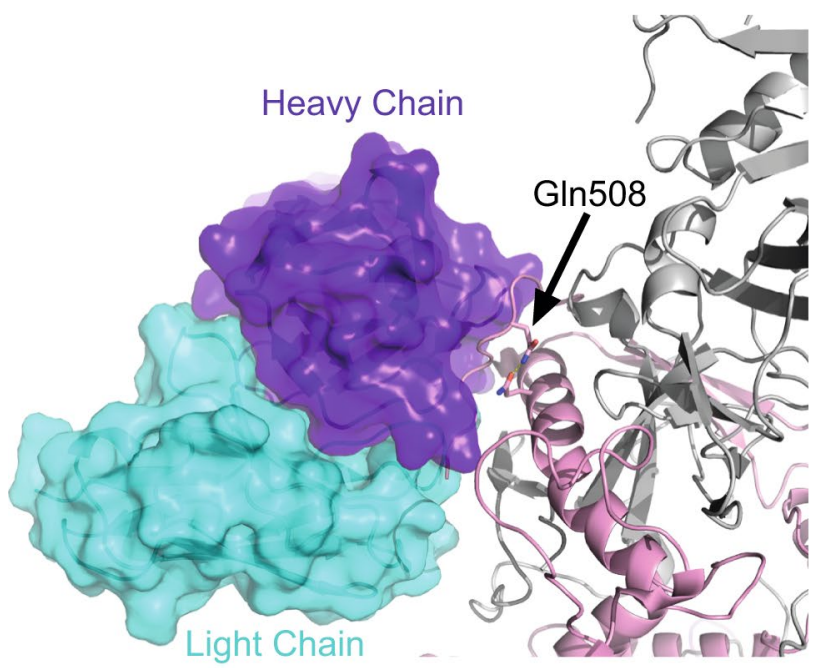

GP2

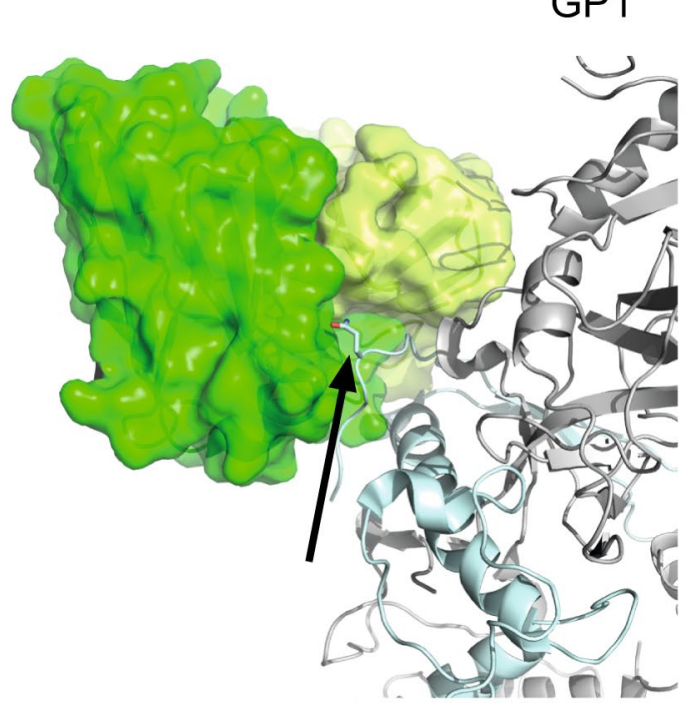

GP2 b

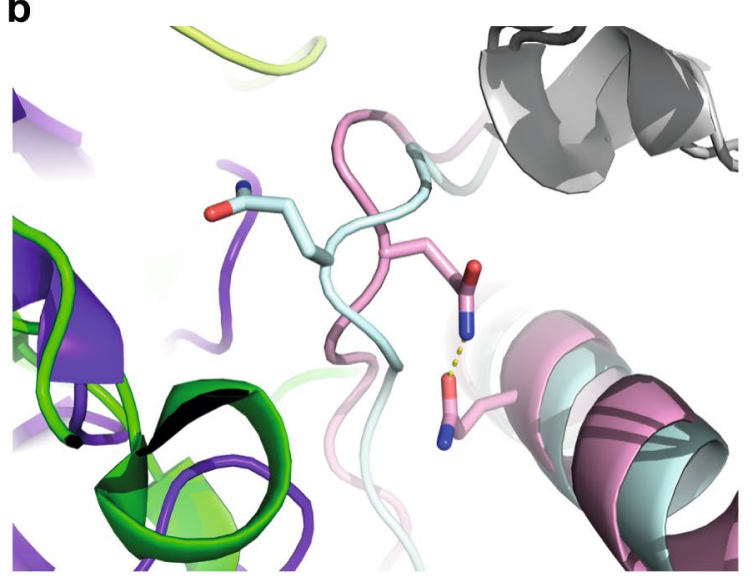

C

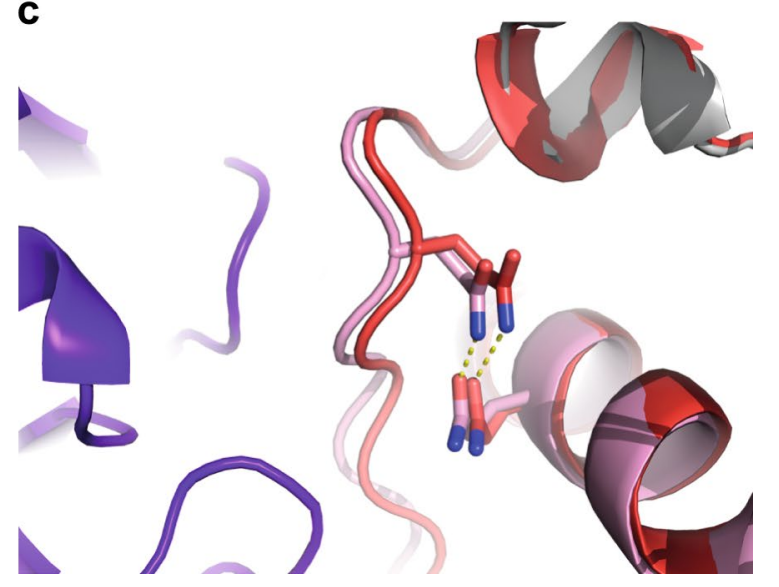

Extended Data Fig. 9 | The role of EBOV GP-GIn508 for the binding of 3 T0331 and C2G4 antibodies (related to Fig. 6). (a) The structures of EBOV GP bound by 3 T0331 (left image), and by C2G4 (right image, PDB: 5KEL). The two antibodies are shown using surface representation in purple and cyan for 3T0331 and in green and lime for C2G4. The heavy and light chains as well as GIn508 that is part of the GP2 portion from the EBOV GP are indicated. The epitopes of these antibodies only partially overlap and only C2G4 binds the side chain of GIn508. (b) A close-up view of the N-terminal parts of GP2 superimposed on each other, using the same colors as in (a). GIn508 in the case of EBOV/3T0331 complex (pink) is pointing away from the antibody and is making a hydrogen bond with Gln560, which is a nearby residue from GP2. In the case of EBOV/C2G4 (light blue), the side chain of Gln508 points toward the antibody and makes important part of its epitope. (c) Superimposition of EBOV/3T0331. Same colors as in (a) with antibody-free structure of EBOV GP (PDB: 5JQ3, shown in red). The N-terminal conformation of GP2 that 3 T0331 recognizes is almost identical to the conformation observed in the native antibody-free state of EBOV GP. 
a

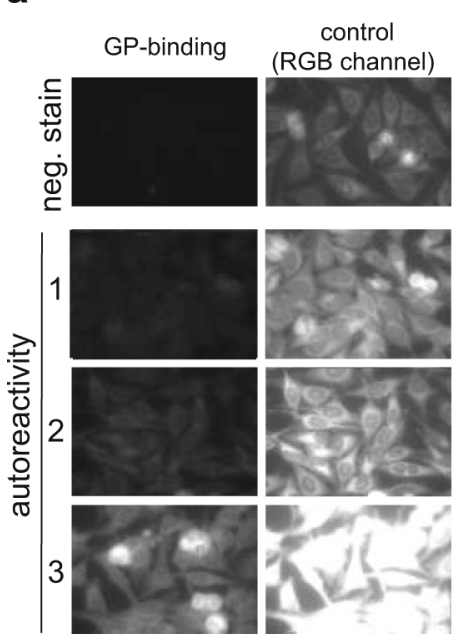

b

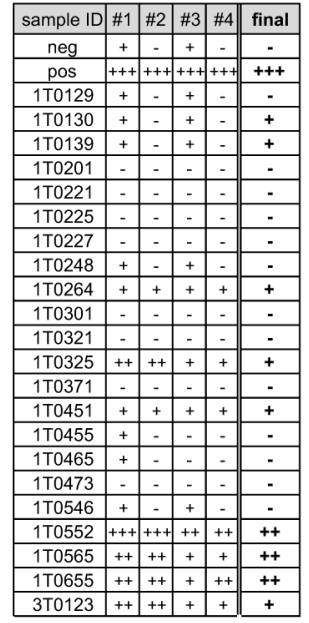

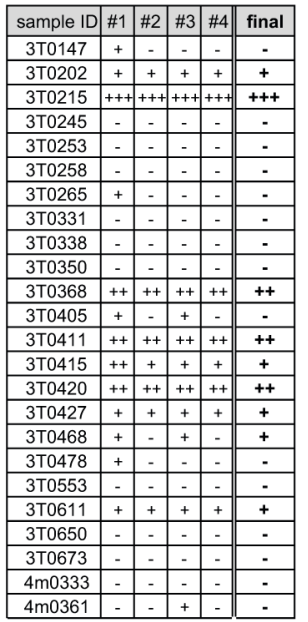

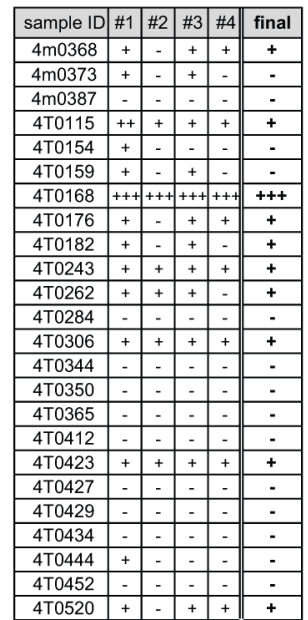

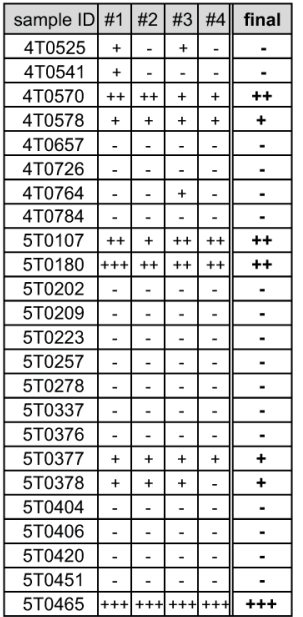

C
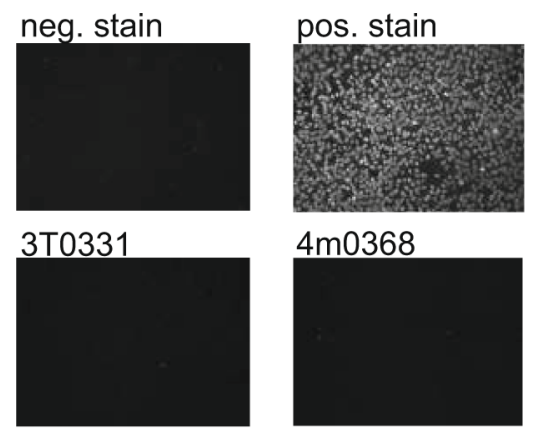

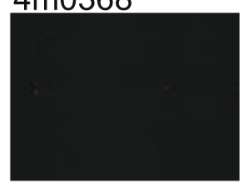

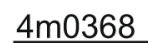

d

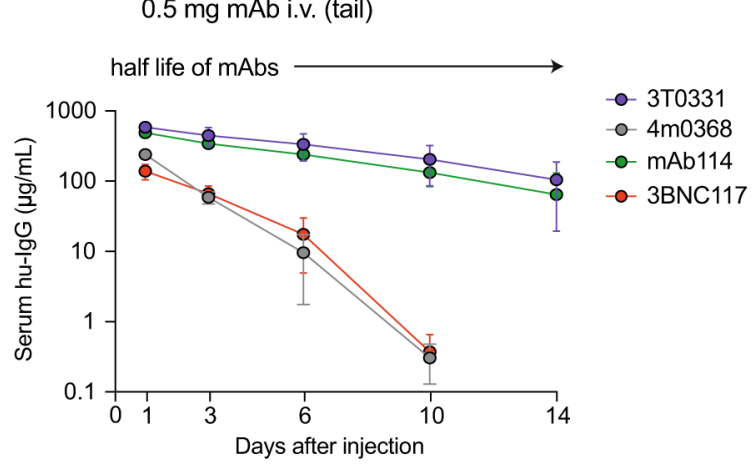

Extended Data Fig. 10 | Autoreactivity and pharmacokinetic assessment of rVSV-ZEBOV-induced antibodies (related to Fig. 6). (a) HepG2 cells were stained with rVSV-ZEBOV-induced mAbs and analyzed by fluorescence microscopy (Leica DMI microscope, left). Unfiltered fluorescence (RGB) was used to control for presence of cells (right). Representative pictures of the scoring system are shown. (b) Autoreactivity signal was categorized into a scoring system ranging from 0 to 3 and quantification of fluorescence signal in a blinded fashion by four independent persons. All assays were performed in duplicates. (c) Representative autoreactivity staining of the mAbs 3 T0331 and $4 \mathrm{m0368}$ of two independent experiments. (d) Pharmacokinetic profile of 3 T0331 and $4 \mathrm{m0368}$ in NRG mice ( $n=3$ for each mAb). $0.5 \mathrm{mg} \mathrm{mAb}$ were injected intravenously (i.v.) and blood samples were taken after different periods of time. As a reference, mAb114 (EBOV GP-specific) and 3BNC117 (HIV-1 Env-specific) were used. 


\section{natureresearch}

Corresponding author(s): Florian Klein, M.D., M.Sc.

Last updated by author(s): Aug 29, 2019

\section{Reporting Summary}

Nature Research wishes to improve the reproducibility of the work that we publish. This form provides structure for consistency and transparency in reporting. For further information on Nature Research policies, see Authors \& Referees and the Editorial Policy Checklist.

\section{Statistics}

For all statistical analyses, confirm that the following items are present in the figure legend, table legend, main text, or Methods section.

$\mathrm{n} / \mathrm{a}$ Confirmed

Х The exact sample size $(n)$ for each experimental group/condition, given as a discrete number and unit of measurement

$\square$ A statement on whether measurements were taken from distinct samples or whether the same sample was measured repeatedly

$\varnothing$ The statistical test(s) used AND whether they are one- or two-sided

Only common tests should be described solely by name; describe more complex techniques in the Methods section.

$\bigotimes \square$ A description of all covariates tested

$\square$ A description of any assumptions or corrections, such as tests of normality and adjustment for multiple comparisons

$\square$ A full description of the statistical parameters including central tendency (e.g. means) or other basic estimates (e.g. regression coefficient)

AND variation (e.g. standard deviation) or associated estimates of uncertainty (e.g. confidence intervals)

For null hypothesis testing, the test statistic (e.g. $F, t, r$ ) with confidence intervals, effect sizes, degrees of freedom and $P$ value noted

Give $P$ values as exact values whenever suitable.

Х $\square$ For Bayesian analysis, information on the choice of priors and Markov chain Monte Carlo settings

$\square$ For hierarchical and complex designs, identification of the appropriate level for tests and full reporting of outcomes

Х $\square$ Estimates of effect sizes (e.g. Cohen's $d$, Pearson's $r$ ), indicating how they were calculated

Our web collection on statistics for biologists contains articles on many of the points above.

\section{Software and code}

Policy information about availability of computer code

Data collection

No data were collected except own data (see also below). Flow cytometry data were gained by FACSArialll with BD FACSDiva software. ELISA plates were measured by Tecan's Sunrise absorbance microplate reader and associated software. Microscopic pictures were taken with Leica DMI microscope (fluorescence) and a Titan Krios electron microscope (FEI) operating at $300 \mathrm{kV}$ (cryo-EM). X-ray diffraction data were collected at the European Synchrotron Radiation Facility (ESRF) at beamline ID23-2 using a Pilatus3 2M detector. B cell receptor repertoire sequence data were generated by an unbiased template-switch-based NGS approach (Kreer et al., manuscript in preparation). Raw read pre-processing was performed with an in-house pipeline primarily based on self-written Python scripts, IgBLAST, Clustal Omega, and the pRESTO toolkit. Antibody sequence data were gained by PCR sequencing at GATC or Eurofins genomics.

Data analysis

Antibody sequence analysis: Sequencing chromatograms were filtered for a mean Phred score of 28 and a minimal length of $240 \mathrm{nt}$. Remaining sequences were annotated with IgBLAST (Ye et al., 2013) and trimmed to extract only the variable region from FWR1 to the end of the J gene. Base calls within the variable region with a Phred score below 16 were masked and sequences with more than 15 masked nucleotides, stop codons, or frameshifts were excluded from further analyses. In order to identify clonally related sequences within a single subject, we grouped all productive heavy chain sequences of that particular subject by identical VH genes, determined the pairwise Levenshtein distance for their $\mathrm{CDRH} 3 \mathrm{~s}$, and assigned an individual clone-number to sequence groups that share the same $\mathrm{VH}$ gene and have a minimal CDRH3 identity of $75 \%$ (with respect to the shortest CDRH3). 100 rounds of input sequence randomization and clonal assignment were performed and the result with the lowest number of remaining unassigned (non-clonal) sequences was selected for downstream analyses.

NGS sequence processing: Raw read pre-processing was performed with an in-house pipeline primarily based on self-written Python scripts, IgBLAST (Ye et al., 2013), Clustal Omega (Sievers et al., 2011), and the pRESTO toolkit (Vander Heiden et al., 2014). Raw reads were filtered for a mean Phred quality score of 25 and a mean read length of $250 \mathrm{bp}$. Unique molecular identifiers (UMIs) were extracted and IgBLAST was used to pre-annotate reads. Each read pair was annotated with its top V gene call, its UMI, and an additional $18 \mathrm{nt}$ molecular identifier (MID) starting $12 \mathrm{nt}$ after the framework region (FWR) 3. UMIs were grouped and sequences (referred to as "collisions") were removed if they differed from the most abundant $V$ gene call or had more than $1 \mathrm{nt}$ difference to the remaining reads in their UMI group. We assumed that reads with the same V gene/MID and an UMI edit distance of 1 nt are more likely to represent RT, 
PCR, or sequencing errors within the UMI than real different molecules. In order to rescue those erroneous UMIs, we re-grouped colliding reads and remaining single UMI reads by their MID and re-defined MID groups as the same UMI group, if they shared the same $\checkmark$ gene and their UMI had no more than $1 \mathrm{nt}$ difference. UMI groups were aligned with clustal omega and then collapsed to build consensus reads. To this end, we weighted each base call by its quality ( 1 - error probability) and generated sums for different base calls over each position to account for both, total abundance and the corresponding quality. The base with the highest sum was taken as the consensus base. Paired consensus reads were assembled to one sequence using pRESTOs AssemblePairs module with a minimal overlap of $6 \mathrm{nt}$. Assembled full-length sequences were annotated with IgBLAST and only productive sequences with full sequence annotation were kept for downstream analysis. $\vee$ gene usage, CDR3 length and $V$ gene germline identity distributions were determined from all final sequences without further collapsing.

Cryo-EM data analysis: The entire data processing was conducted using the cryoSPARC V2 suite (Punjani et al., 2017). Movies were motion-corrected and contrast transfer functions were fitted using CTFFIND4 (Rohou and Grigorieff, 2015). Templates for auto picking were derived by 2D classification of manually picked particles. Following template-based autopicking a total of 109,407 and 123,880 good particles were selected for reconstruction of EBOV GP/3T0331 and EBOV GP/4m0368, respectively based on iterative reference-free 2D classifications. Initial reference maps were calculated using Ab-initio reconstruction and high-resolution maps were obtained using the non-uniform 3D refinement, while imposing C3 symmetry. Working maps were locally filtered after calculating local resolution estimates.

Analysis of antibody protein sequences: The calculations of phylogenetic trees and sequence alignments were done with Geneious (v10). Sequence similarity of $\mathrm{CDRH} 3$ was also determined by Geneious software using BLOSUM (BLOcks Substitution Matrix) with residuespecific and hydrophilic gap penalties (open gap penalty of 10 , extended gap penalty 0.2 . For comparing the heterogeneity of sequences within the same donor with that among different donors, pairwise alignment scores among all sequences were computed by needle (with default parameters) and grouped accordingly. The difference of both distribution of alignment scores was assessed with a MannWhitney-U test. Sequence logo plots were generated with WebLogo3 followed by minor adjustments.

Flow cytometry, ELISA and statistical analysis: Flow cytometry analyses and quantifications were performed with FlowJo10. EC50 values, means and standard deviations of serum and antibody reactivities were calculated from technical replicates using GraphPad Prism ( 7 ). Tests for significance were conducted using GraphPad Prism and R (R Core Team; 2018).

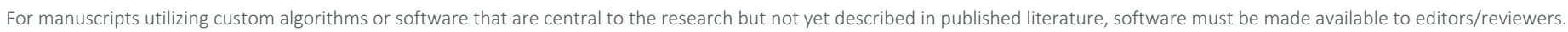
We strongly encourage code deposition in a community repository (e.g. GitHub). See the Nature Research guidelines for submitting code \& software for further information.

Data

Policy information about availability of data

All manuscripts must include a data availability statement. This statement should provide the following information, where applicable:

- Accession codes, unique identifiers, or web links for publicly available datasets

- A list of figures that have associated raw data

- A description of any restrictions on data availability

In the manuscript we present the data necessary to interpret and replicate our results. All experiments were performed as described in the method section. Al antibody sequences are currently being processed to be uploaded to GenBank.

EM structures have been deposited at PDB and are hold until final publication.

\section{Field-specific reporting}

Please select the one below that is the best fit for your research. If you are not sure, read the appropriate sections before making your selection.

$\bigotimes$ Life sciences

Behavioural \& social sciences

Ecological, evolutionary \& environmental sciences

For a reference copy of the document with all sections, see nature.com/documents/nr-reporting-summary-flat.pdf

\section{Life sciences study design}

All studies must disclose on these points even when the disclosure is negative.

Sample size

Sample size of investigated subjects was chosen based on previous studies (Davis et al., Cell 2019; Corti et al., Science 2016; Klein et al., J Exp med. 2012) to sufficiently inform on proof of concept.

Data exclusions Sequencing chromatograms were filtered for a mean Phred score of 28 and a minimal length of 240 nt. Remaining sequences were annotated with IgBLAST (Ye et al., 2013) and trimmed to extract only the variable region from FWR1 to the end of the J gene. Base calls within the variable region with a Phred score below 16 were masked and sequences with more than 15 masked nucleotides, stop codons, or frameshifts were excluded from further analyses. Exclusion criteria were not pre-established and first used in this study.

Replication

To test reproducibility experiments were repeated with the same setup at least two times. The results of single cell sort were verified by repeating the flow cytometric staining at least one time (except for EV07 due to low amount of samples). All attempts of replication were successful.

Randomization Randomization was not applicable in this study. 


\section{Reporting for specific materials, systems and methods}

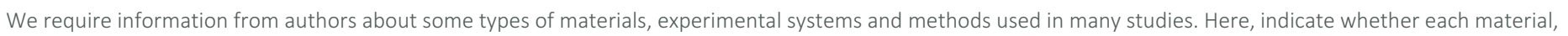

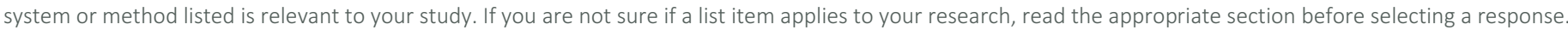

\begin{tabular}{l|l} 
Materials \& experimental syste & \multicolumn{1}{l}{ Involved in the study } \\
\hline $\mathrm{n} / \mathrm{a}$ & $\bigotimes$ Antibodies \\
$\square$ & $\square$ Eukaryotic cell lines \\
$\square$ & $\bigotimes$ Animals and other organisms \\
$\square$ & $\bigotimes$ Human research participants \\
$\square$ & $\bigotimes$ Clinical data
\end{tabular}

\begin{tabular}{l|l} 
Methods \\
\hline n/a Involved in the study \\
$\square$ \\
$\square$ ChIP-seq \\
$\square$ & $\square$ Flow cytometry \\
$\square$ MRI-based neuroimaging
\end{tabular}

Antibodies

Antibodies used

1.) Mouse anti-human CD20-AF700 (clone 2H7), BD Bioscience, Cat\#560631, RRID: AB_2687799,

2.) Mouse anti-human IgG-APC (clone G18-145), BD Bioscience, Cat\#550931, RRID: AB_2738854,

3.) Goat anti-human IgG-HRP, Southern Biotech, Cat\# 2040-05,

4.) Human anti-EBOV GP ADI-15758, GenBank: KU602137-8,

5.) Human anti-EBOV GP ADI-15999, GenBank: KU602605-6,

6.) Human anti-EBOV GP ADI-16037, GenBank: KU602673-4,

7.) Human anti-EBOV GP mAb100, PDB: $5 F H B$,

8.) Human anti-EBOV GP mAb114, PDB: 5FHA,

9.) Human anti-EBOV GP KZ52, PDB: 3 INU

10.) FITC-conjugated anti-human IgG (NOVA Lite HEp-2 ANA Kit; Inova Diagnostics)

Validation

1.) Mouse anti-human CD20-AF700 (clone $2 \mathrm{H} 7$ ): validated by Flow cytometry

2.) Mouse anti-human IgG-APC (clone G18-145): validated by Flow cytometry

3.) Goat anti-human IgG-HRP, Southern Biotech: validated by ELISA, FLISA and flow cytometry

4.) Human anti-EBOV GP ADI-15758, GenBank: KU602137-8: published in Rapid and large-scale isolation of potent neutralizing antibodies from a survivor of the 2014 Ebola virus outbreak; Bornholdt et al.; Science (2016)

5.) Human anti-EBOV GP ADI-15999, GenBank: KU602605-6, published in Rapid and large-scale isolation of potent neutralizing antibodies from a survivor of the 2014 Ebola virus outbreak; Bornholdt et al.; Science (2016)

6.) Human anti-EBOV GP ADI-16037, GenBank: KU602673-4, published in Rapid and large-scale isolation of potent neutralizing antibodies from a survivor of the 2014 Ebola virus outbreak; Bornholdt et al.; Science (2016)

7.) Human anti-EBOV GP mAb100, PDB: 5FHB, published in Structural and molecular basis for Ebola virus neutralization by protective human antibodies; Misasi, J. et al.; Science (2016)

8.) Human anti-EBOV GP mAb114, PDB: 5FHA, published in Structural and molecular basis for Ebola virus neutralization by protective human antibodies; Misasi, J. et al.; Science (2016)

9.) Human anti-EBOV GP KZ52, PDB: 3INU, published in Techniques and tactics used in determining the structure of the trimeric ebolavirus glycoprotein; Lee, J.E. et al.; Acta Crystallogr. (2009)

10.) FITC-conjugated anti-human IgG (NOVA Lite HEp-2 ANA Kit; Inova Diagnostics) validated by Assay quality control

\section{Eukaryotic cell lines}

Policy information about cell lines

Cell line source(s)

HEK293T (ATCC); HEK293E (ATCC); HEK293F (National Research Council Canada), FreeStyle ${ }^{\text {TM }}$ 293-F Cells (Thermo Fisher Scientific); Vero C1008 (ATCC); HepG2 cells (NOVA Lite HEp-2 ANA Kit; Inova Diagnostics)

\section{Authentication}

Cell lines were not authentificated.

Mycoplasma contamination

Cell lines were tested negative for mycoplasma contamination.

Commonly misidentified lines

(See ICLAC register)

In this study no commonly misidentified cell lines were used. 
Policy information about studies involving animals; ARRIVE guidelines recommended for reporting animal research

Laboratory animals

Wild animals

Field-collected samples

Ethics oversight
NOD.Cg-Rag1tm1Mom II2rgtm1Wjl/SzJ (The Jackson Laboratory). Each group contained two female and one male with age between 22-36 weeks.

This study did not involve wild animals.

This study did not involve samples collected from the field.

LANUV

Note that full information on the approval of the study protocol must also be provided in the manuscript.

\section{Human research participants}

\section{Policy information about studies involving human research participants}

Population characteristics All individuals participated in the Phase I Trial to Assess the Safety, Tolerability and Immunogenicity of an Ebola Virus Vaccine (rVSVAG-ZEBOV-GP; NCT02283099) and were vaccinated with either $3 \times 10^{\wedge} 5$ (EV01, EV02, EV04 and EV06) or $3 \times 106$ pfu (EV03 and EV05). The age of the selected individuals was 55, 26, 42, 51, 44 and 45 years (EV01-EV06). Two of the individuals were female (EV01 and EV02). Samples were taken after 18.5, 18.5, 24, 20.5, 25 and 26 month after vaccination (EV01-EV06). EV07 is a health care worker (age 47) that was vaccinated with $2 \times 10^{\wedge} 7$ in December 2018.

Recruitment

Individuals studied were previously enrolled in the Phase I Trial to Assess the Safety, Tolerability and Immunogenicity of an Ebola Virus Vaccine (rVSVAG-ZEBOV-GP; NCT02283099) and were vaccinated with either $3 \times 105$ or $3 \times 106$ pfu. From this cohort, six individuals were enrolled in an observational study (INA; 16-054) at the University Hospital of Cologne to collect serum and PBMC samples. Recruitment was open for all participants in trial NCT02283099 that were enrolled at the site in Hamburg. All participants interested in our study were enrolled. No potential self-selection bias or other bias are known.

Ethics oversight

The protocol was approved by the Institutional Review Board (IRB) of the University of Cologne, Germany.

Note that full information on the approval of the study protocol must also be provided in the manuscript.

\section{Clinical data}

Policy information about clinical studies

All manuscripts should comply with the ICMJE guidelines for publication of clinical research and a completed CONSORT checklist must be included with all submissions.

Clinical trial registration

https://www.viomedo.de/klinische-studien/6383/identifikation-neutralisierender-antikoerper-chronische-schwerwiegendeinfektionen

Study protocol

https://www.viomedo.de/klinische-studien/6383/identifikation-neutralisierender-antikoerper-chronische-schwerwiegendeinfektionen

Data collection

Data collection was performed at enrollement after informed consent.

Outcomes

Non-Interventional study. No outcome paramenters were defined except number of collected samples.

\section{Flow Cytometry}

Plots

Confirm that:

Х The axis labels state the marker and fluorochrome used (e.g. CD4-FITC).

Х The axis scales are clearly visible. Include numbers along axes only for bottom left plot of group (a 'group' is an analysis of identical markers).

$\square$ All plots are contour plots with outliers or pseudocolor plots.

$\bigotimes$ A numerical value for number of cells or percentage (with statistics) is provided.

\section{Methodology}

Sample preparation
Peripheral blood mononuclear cells (PBMCs) were enriched for B lymphocytes by magnetic cell separation (MACS) with CD19microbeads (Miltenyi Biotec) according to manufacturer's instruction. MACS LS separation columns (Miltenyi Biotec) were used to enrich labeled cells. B cells were spun and suspended in PBS (Gibco) with 2\% FBS (Merck) and 2 mM EDTA (Thermo Fisher Scientific) and subjected to fluorescence staining with DAPI (Thermo Fisher Scientific), anti-huCD20-Alexa Fluor 700 (BD), antihulgG-APC (BD), and EBOV GP $\triangle T$ TM-DyLight 488 (Microscale Antibody Kit, Thermo Fisher Scientific). Cells were stained for 20 min at $4^{\circ} \mathrm{C}$ and live $\mathrm{CD} 20+\lg \mathrm{G}+\mathrm{EBOV} \mathrm{GP}+$ cells were sorted. 
Instrument

Software

Cell population abundance

Gating strategy
FACSArialll (Becton Dickinson) in a single cell manner into 96-well plates.

BD FACSDIVA ${ }^{\text {TM }}$ SOFTWARE

Described in Figure 1.

Gating strategies are shown in Fig 1 and Supplementary Fig. 2.

$\bigotimes$ Tick this box to confirm that a figure exemplifying the gating strategy is provided in the Supplementary Information. 